\title{
Module Extraction in Expressive Ontology Languages via Datalog Reasoning
}

\author{
Ana Armas Romero \\ Mark Kaminski \\ Bernardo Cuenca Grau \\ Ian Horrocks \\ Department of Computer Science, \\ University of Oxford, \\ Wolfson Building, Parks Road, \\ Oxford, OX1 3QD, UK
}

\author{
ANA.ARMAS@CS.OX.AC.UK \\ MARK.KAMINSKI@CS.OX.AC.UK \\ BERNARDO.CUENCA.GRAU@CS.OX.AC.UK \\ IAN.HORROCKS@CS.OX.AC.UK
}

\begin{abstract}
Module extraction is the task of computing a (preferably small) fragment $\mathcal{M}$ of an ontology $\mathcal{O}$ that preserves a class of entailments over a signature of interest $\Sigma$. Extracting modules of minimal size is well-known to be computationally hard, and often algorithmically infeasible, especially for highly expressive ontology languages. Thus, practical techniques typically rely on approximations, where $\mathcal{M}$ provably captures the relevant entailments, but is not guaranteed to be minimal. Existing approximations ensure that $\mathcal{M}$ preserves all second-order entailments of $\mathcal{O}$ w.r.t. $\Sigma$, which is a stronger condition than is required in many applications, and may lead to unnecessarily large modules in practice. In this paper we propose a novel approach in which module extraction is reduced to a reasoning problem in datalog. Our approach generalises existing approximations in an elegant way. More importantly, it allows extraction of modules that are tailored to preserve only specific kinds of entailments, and thus are often significantly smaller. Our evaluation on a wide range of ontologies confirms the feasibility and benefits of our approach in practice.
\end{abstract}

\section{Introduction}

Module extraction is the task of computing, given an ontology $\mathcal{O}$ and a signature of interest $\Sigma$, a (preferably small) subset $\mathcal{M}$ of $\mathcal{O}$ (a module) that preserves the class of $\Sigma$-entailments of $\mathcal{O}$ relevant to the application at hand. Such a module is therefore indistinguishable from $\mathcal{O}$ w.r.t. relevant $\Sigma$-entailments, and the application can safely rely on $\mathcal{M}$ instead of $\mathcal{O}$ for all tasks that concern only symbols from $\Sigma$.

Module extraction has received a great deal of attention in recent years (Seidenberg \& Rector, 2006; Stuckenschmidt, Parent, \& Spaccapietra, 2009; Cuenca Grau, Horrocks, Kazakov, \& Sattler, 2008; Kontchakov, Wolter, \& Zakharyaschev, 2010; Del Vescovo, Parsia, Sattler, \& Schneider, 2011; Nortje, Britz, \& Meyer, 2013; Gatens, Konev, \& Wolter, 2014). Modules have found numerous applications in ontology reuse (Cuenca Grau et al., 2008; Jiménez-Ruiz, Cuenca Grau, Sattler, Schneider, \& Berlanga Llavori, 2008), matching (Jiménez-Ruiz \& Cuenca Grau, 2011), debugging (Suntisrivaraporn, Qi, Ji, \& Haase, 2008; Ludwig, 2014) and classification (Armas Romero, Cuenca Grau, \& Horrocks, 2012; Tsarkov \& Palmisano, 2012; Cuenca Grau, Halaschek-Wiener, Kazakov, \& Suntisrivaraporn, 2010). 
The preservation of relevant entailments is formalised via inseparability relations (Konev, Lutz, Walther, \& Wolter, 2009). The strongest such notion is model inseparability, which requires that it must be possible to turn any model of $\mathcal{M}$ into a model of $\mathcal{O}$ by (re-)interpreting only symbols outside $\Sigma$; in this case, $\mathcal{M}$ preserves all second-order $\Sigma$-entailments of $\mathcal{O}$ (Konev, Lutz, Walther, \& Wolter, 2013). A weaker and more flexible notion is that of deductive inseparability, which only requires $\mathcal{O}$ and $\mathcal{M}$ to entail the same $\Sigma$-formulas in a particular query language. Unfortunately, the decision problems associated with module extraction are generally of high complexity or even undecidable, especially for expressive ontology languages. For model inseparability, checking whether $\mathcal{M}$ is a module of $\mathcal{O}$ w.r.t. $\Sigma$ is undecidable even if $\mathcal{O}$ is restricted to the lightweight description logic (DL) $\mathcal{E} \mathcal{L}$ (Konev et al., 2013), for which standard reasoning is tractable (Baader, Brandt, \& Lutz, 2005). For deductive inseparability, the problem is typically decidable for lightweight DLs and "reasonable" query languages, albeit still of high worst-case complexity; for instance, it is ExPTIME-complete for $\mathcal{E} \mathcal{L}$ if we consider concept inclusions as the query language (Lutz $\&$ Wolter, 2010). Practical algorithms that ensure minimality of the extracted modules are known only for $\mathcal{E} \mathcal{L} \mathcal{I}$ ontologies satisfying a particular acyclicity condition (Konev et al., 2013) as well as for dialects of DL-Lite (Kontchakov et al., 2010). To the best of our knowledge, the complexity of module extraction for ontology languages that are not based on DLs, such as variants of datalog ${ }^{ \pm}$(Calì, Gottlob, Lukasiewicz, Marnette, \& Pieris, 2010), remains largely unexplored.

Practical module extraction techniques are typically based on sound approximations, which ensure that the computed fragment $\mathcal{M}$ is a module (i.e., inseparable from $\mathcal{O}$ w.r.t. $\Sigma$ ), but provide no minimality guarantee. The most popular such techniques are based on a family of polynomially checkable conditions based on the notion of syntactic locality (Cuenca Grau, Horrocks, Kazakov, \& Sattler, 2007a; Cuenca Grau et al., 2008; Sattler, Schneider, \& Zakharyaschev, 2009). Each locality-based module $\mathcal{M}$ enjoys a number of desirable properties w.r.t. the signature $\Sigma$ of interest:

(P1) It is model inseparable from $\mathcal{O}$, thus preserving all second-order $\Sigma$-entailments of $\mathcal{O}$.

(P2) It is depleting, in the sense that $\mathcal{O} \backslash \mathcal{M}$ is inseparable from the empty ontology; this implies that no relevant information is "left behind" after extracting $\mathcal{M}$ from $\mathcal{O}$.

(P3) It is self-contained, in that it preserves not only the relevant entailments w.r.t. $\Sigma$, but also w.r.t. all other symbols in its signature.

(P4) It is justification-preserving, in the sense that each subset-minimal fragment of $\mathcal{O}$ preserving a $\Sigma$-entailment (each justification) is contained in $\mathcal{M}$.

(P5) It can be computed efficiently, even for ontologies in expressive description logics.

Model inseparability ensures that modules can be used regardless of the query language relevant to the application at hand. Depletingness and self-containment have been identified as important properties for ontology reuse and modular ontology development tasks (Sattler et al., 2009; Jiménez-Ruiz et al., 2008). Finally, the preservation of justifications enables the use of modules for optimising debugging and explanation services (Schlobach \& Cornet, 2003; Kalyanpur, Parsia, Horridge, \& Sirin, 2007), as well as incremental reasoning (Suntisrivaraporn, 2008; Cuenca Grau et al., 2010). 
Locality-based module extraction techniques are easy to implement, and surprisingly effective in practice. Their main drawback is that the extracted modules can be rather large, which limits their usefulness in some applications (Del Vescovo, Klinov, Parsia, Sattler, Schneider, \& Tsarkov, 2013). One way to address this issue is to develop techniques that approximate minimal modules more closely, while still fulfilling properties $(\mathrm{P} 1)-(\mathrm{P} 4)$. Efforts in this direction have confirmed that locality-based modules can be far from optimal in practice (Gatens et al., 2014); however, these techniques apply only to rather restricted ontology languages and utilise algorithms with high worst-case complexity.

Another approach to computing smaller modules is to weaken properties (P1)-(P4), which are stronger than many applications require. In particular, model inseparability is a very strong condition, and deductive inseparability w.r.t. a query language suitable for the application at hand would usually suffice.

In this paper, we propose a novel approach that reduces module extraction to a reasoning problem in the basic rule-based language datalog (Abiteboul, Hull, \& Vianu, 1995; Dantsin, Eiter, Gottlob, \& Voronkov, 2001). The connection between module extraction and datalog was first observed by Suntisrivaraporn (2008), who showed that locality $\perp$-module extraction for $\mathcal{E} \mathcal{L}$ ontologies could be reduced to propositional datalog reasoning. Our approach takes this connection much farther, and generalises locality-based modules in an elegant way. The key distinguishing features of our approach are as follows:

- It is applicable not only to ontology languages based on description logics, but also to expressive rule-based knowledge representation formalisms that extend datalog with existential quantification and disjunction in the head of rules (Calì et al., 2010; Bourhis, Morak, \& Pieris, 2013; Alviano, Faber, Leone, \& Manna, 2012).

- It is sensitive to the different inseparability relations proposed in the literature; in particular, we can extract deductively inseparable modules with the query language tailored to the specific requirements of the application at hand. This allows us to relax property (P1) and extract significantly smaller modules.

- In all cases, our modules are depleting and capture all justifications of relevant entailments; moreover, our approach can be adapted to either ensure or dispense with self-containment, depending on the application needs.

- It not only ensures tractability of module extraction for DL-based ontology languages, but also enables the use of highly scalable off-the-shelf datalog reasoners.

We have implemented our approach using the RDFox datalog reasoner (Motik, Nenov, Piro, Horrocks, \& Olteanu, 2014). Our evaluation over complex, real-world, ontologies shows that module size consistently decreases as we consider weaker inseparability relations, which could significantly improve the usefulness of modules in applications.

\section{Preliminaries}

In Section 2.1 we introduce the language of first-order rules, which is powerful enough to fully capture expressive rule-based ontology languages such as datalog ${ }^{ \pm}$(Calì et al., 2010), and datalog ${ }^{ \pm, \vee}$ (Bourhis et al., 2013; Alviano et al., 2012), as well as all mainstream 
description logics (Baader, Calvanese, McGuinness, Nardi, \& Patel-Schneider, 2003). Most of our results in this paper hold for arbitrary knowledge bases consisting of such first-order rules, and hence are applicable to a very wide range of knowledge representation formalisms.

In Section 2.2 we introduce the syntax and first-order semantics of the description logic $\mathcal{S R O I Q}$ (Horrocks, Kutz, \& Sattler, 2006), which underpins the W3C standard ontology language OWL 2 (Motik, Patel-Schneider, \& Parsia, 2012; Cuenca Grau, Horrocks, Motik, Parsia, Patel-Schneider, \& Sattler, 2008). We then introduce a normal form for $\mathcal{S R O I \mathcal { L }}$ and establish its correspondence to first-order rules. Finally, in Section 2.3 we briefly recall the well-known hyperresolution calculus for first-order logic (Bachmair \& Ganzinger, 2001), which we exploit in many of our technical results to show that a module preserves the required consequences of the given ontology.

Throughout this paper, we assume basic familiarity with first-order logic and we use standard first-order logic notions, such as predicates, constants, variables, terms, atoms, formulas, sentences, interpretations and entailment (written $=$ ). We define a signature as a set of predicates; furthermore, given a first-order sentence $\phi$, we use $\operatorname{Sig}(\phi)$ to denote the signature of $\phi$. We then say that $\phi$ is a $\Sigma$-sentence if $\operatorname{Sig}(\phi) \subseteq \Sigma$. Analogously, we denote with $\operatorname{Ct}(\phi)$ the set of constants in $\phi$. These definitions extend naturally to sets of sentences; indeed, later on in the paper we will speak of $\Sigma$-rules, $\Sigma$-datasets, and $\Sigma$-ontologies with the obvious meaning. The restriction of signatures to contain just predicates and the separate treatment of constants will be convenient for working with inseparability relations later on.

A set of function-free sentences $\mathcal{F}^{\prime}$ is a (model) conservative extension of a set $\mathcal{F}$ if for each model $\mathcal{I}$ of $\mathcal{F}$ there is a model $\mathcal{J}$ of $\mathcal{F}^{\prime}$ with the same domain as $\mathcal{I}$ and such that $A^{\mathcal{I}}=A^{\mathcal{J}}$ for each $A \in \operatorname{Sig}(\mathcal{F})$ and $a^{\mathcal{I}}=a^{\mathcal{J}}$ for each $a \in \operatorname{Ct}(\mathcal{F})$.

We deviate slightly from the standard definition of first-order logic in that our definition does not include the nullary symbols $T$ and $\perp$, which are interpreted as true and false respectively in every first-order interpretation. Similarly, we consider first-order logic without equality $\approx$ and hence we do not assume that $\approx$ is interpreted as the identity relation over the domain in every interpretation. Instead, we treat $\perp, \top$ and $\approx$ as ordinary predicates, the meaning of which we axiomatise explicitly in every knowledge base. We assume that $\perp$ is nullary, $T$ is unary and $\approx$ is binary. Given a set $\mathcal{F}$ of function-free sentences, we then define the following sets of sentences $\mathcal{F}^{\perp}, \mathcal{F}^{\top}$, and $\mathcal{F}^{\approx}$.

- $\mathcal{F}^{\perp}$ is empty if $\mathcal{F}$ contains no occurrences of $\perp$, and the singleton set $\{\neg \perp\}$ otherwise.

- $\mathcal{F}^{\top}$ is empty if $\mathcal{F}$ contains no occurrence of $T$; otherwise, it is the set

$$
\left\{\forall x_{1}, \ldots, x_{n}\left[A\left(x_{1}, \ldots, x_{n}\right) \rightarrow \top\left(x_{i}\right)\right] \mid A \in \operatorname{Sig}(\mathcal{F}) n \text {-ary, } 1 \leq i \leq n\right\}
$$

- $\mathcal{F} \approx$ is empty if $\mathcal{F}$ contains no occurrences of $\approx$; otherwise, it consists of the sentences (EQ1)-(EQ5) given next. Sentence (EQ1) is instantiated for each constant $a \in \mathrm{Ct}(\mathcal{F})$; furthermore, sentences (EQ2) and (EQ5) are instantiated for each $n$-ary predicate $A$ 
in $\operatorname{Sig}(\mathcal{F})$ and each $x_{i}$ in $\mathbf{x}=x_{1}, \ldots, x_{n}$ :

$$
\begin{aligned}
& \rightarrow a \approx a \\
\forall \mathbf{x}[A(\mathbf{x}) & \left.\rightarrow x_{i} \approx x_{i}\right] \\
\forall x, y[x \approx y & \rightarrow y \approx x] \\
\forall x, y, z[x \approx y \wedge y \approx z & \rightarrow x \approx z] \\
\forall \mathbf{x}, y\left[A(\mathbf{x}) \wedge x_{i} \approx y\right. & \left.\rightarrow A\left(x_{1}, \ldots, x_{i-1}, y, x_{i+1}, \ldots, x_{n}\right)\right]
\end{aligned}
$$

We consider substitutions as functional mappings between two sets of terms. Given a substitution $\sigma$ and a term $t$ not in the domain of $\sigma$, in an abuse of notation the expression $t \sigma$ denotes $t$. Substitutions can be applied to formulas: given an atom $A\left(t_{1}, \ldots, t_{n}\right)$, $A\left(t_{1}, \ldots, t_{n}\right) \sigma=A\left(t_{1} \sigma, \ldots, t_{n} \sigma\right)$, and given a non-atomic formula $\phi, \phi \sigma$ is the result of applying $\sigma$ to all atoms in $\phi$. This application is extended to sets of formulas in the natural way. Given two substitutions $\sigma$ and $\tau$, their composition is the substitution $\sigma \tau$ such that $t(\sigma \tau)=(t \sigma) \tau$ for each $t$ in the domain of $\sigma$. We say that $\sigma$ and $\tau$ are compatible if they coincide over the intersection of their domains. If $\sigma$ and $\tau$ are compatible, their union is the substitution $\sigma \cup \tau$ such that $t(\sigma \cup \tau)=t \sigma$ for each $t$ in the domain of $\sigma$ and $t(\sigma \cup \tau)=t \tau$ for each $t$ in the domain of $\tau$. Finally, we use $\operatorname{dom}(\sigma)$ (resp. range $(\sigma)$ ) to denote the domain (resp. the range) of $\sigma$.

\subsection{Rule-Based First-Order Languages}

Rule-based languages are prominent knowledge representation formalisms closely related to ontology languages (Dantsin et al., 2001; Bry, Eisinger, Eiter, Furche, Gottlob, Ley, Linse, Pichler, \& Wei, 2007; Calì et al., 2010). In this paper, we focus on monotonic formalisms and hence on rule languages that can be seen as fragments of first-order logic. We next define a general notion of first-order rule which underpins the datalog ${ }^{ \pm}$and datalog $^{ \pm, \vee}$ families of languages (Calì et al., 2010; Alviano et al., 2012).

A fact $\gamma$ is a function-free ground atom. A finite set of facts is called a dataset. A rule $r$ is a function-free first-order sentence of the form

$$
\forall \mathbf{x}[\varphi(\mathbf{x}) \rightarrow \exists \mathbf{y} \psi(\mathbf{x}, \mathbf{y})]
$$

where $\mathbf{x}$ and $\mathbf{y}$ are disjoint vectors of variables, $\varphi$ is a (possibly empty) conjunction of distinct atoms over constants and variables from $\mathbf{x}$; and $\psi$ is built from atoms over constants and variables from $\mathbf{x} \cup \mathbf{y}$ using conjunction $(\wedge)$ and disjunction $(\vee)$. Note that any fact is also a rule. Formula $\varphi$ is the rule body and $\exists \mathbf{y} \psi(\mathbf{x}, \mathbf{y})$ is the rule head. The head of a rule can be empty, in which case we represent it as $\square$. Universal quantifiers in rules are omitted for brevity. Rules are required to be safe, that is, all universally quantified variables in the head must occur in the body. A rule is datalog if its head is either empty or it consists of a single atom where all variables are universally quantified. Note that, for any set $\mathcal{F}$ of function-free sentences, the set $\mathcal{F}^{\perp} \cup \mathcal{F}^{\top} \cup \mathcal{F}^{\approx}$ contains only datalog rules.

A (first-order) ontology $\mathcal{O}$ is a finite set of rules satisfying $\mathcal{O}^{\perp} \cup \mathcal{O}^{\top} \cup \mathcal{O} \approx \subseteq \mathcal{O}$. We assume w.l.o.g. that different rules in $\mathcal{O}$ do not share existentially quantified variables and that the only rule with empty head is $\perp \rightarrow \square$. 


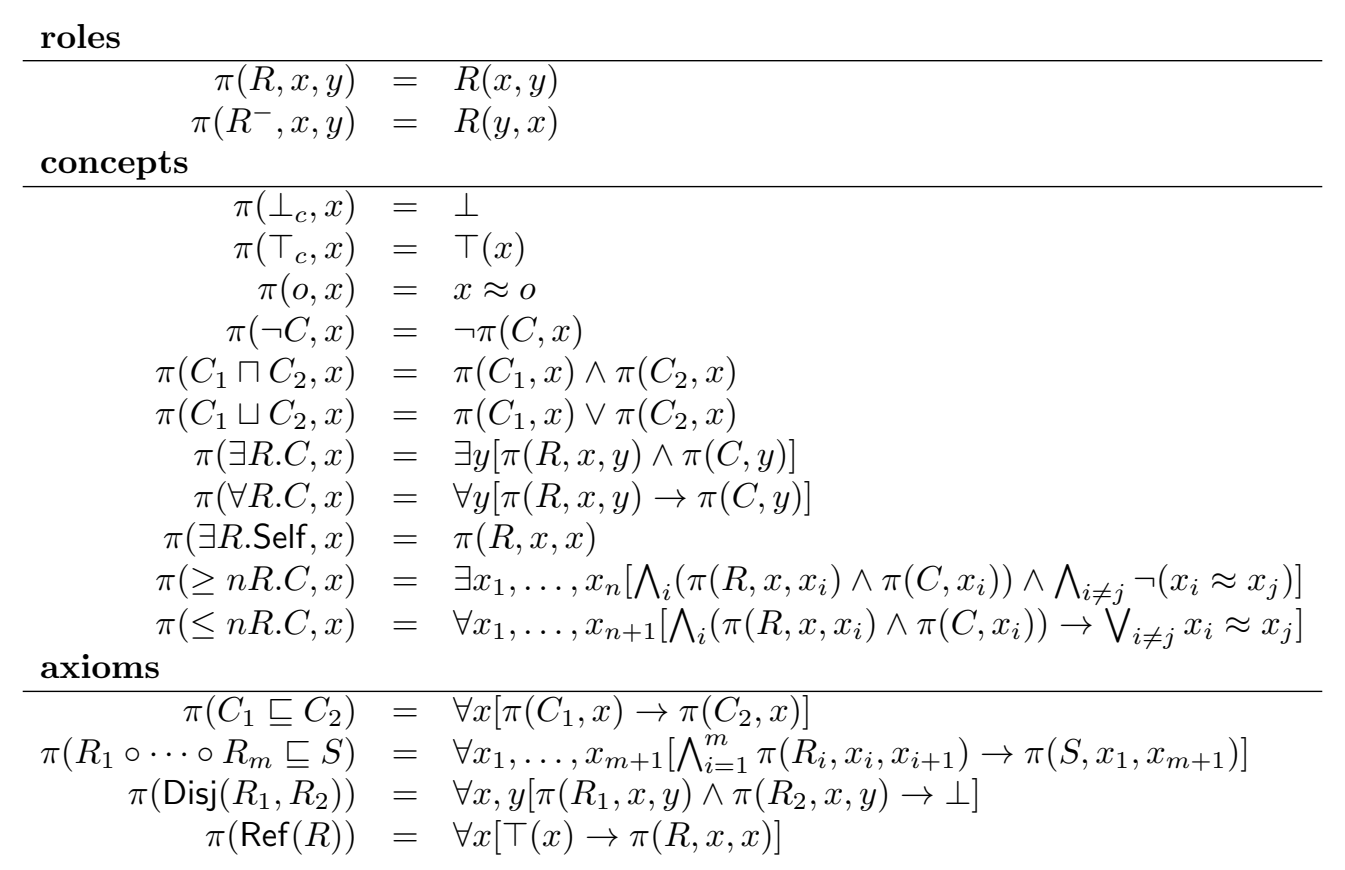

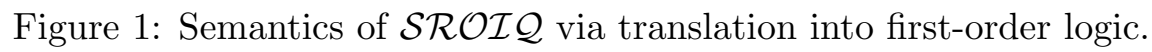

A datalog program is an ontology containing only datalog rules. Given a datalog program $\mathcal{P}$ and a dataset $\mathcal{D}$, their materialisation, denoted with $\mathcal{P}(\mathcal{D})$, is the set of facts entailed by $\mathcal{P} \cup \mathcal{D}$. Such materialisation can be computed in time polynomial in the size of $\mathcal{D}$ using forward chaining (Abiteboul et al., 1995; Dantsin et al., 2001).

To conclude this section, we define the languages typically used for querying first-order ontologies. We define a Boolean positive existential query (Boolean PEQ) as a non-empty sentence $q$ built from function-free atoms using only $\exists, \wedge$ and $\vee$; such a query holds w.r.t. an ontology $\mathcal{O}$ if $\mathcal{O} \models q$. A Boolean PEQ is a conjunctive query (CQ) if it is disjunctionfree. The following proposition, the proof of which is straightforward, establishes a useful connection between Boolean PEQ evaluation and entailment of first-order rules.

Proposition 1. Let $\mathcal{O}$ be an ontology, $r=\bigwedge_{i=1}^{n} \gamma_{i}(\mathbf{x}) \rightarrow \exists \mathbf{y} \psi(\mathbf{x}, \mathbf{y})$ a rule, and let $\sigma$ be a substitution mapping all universally quantified variables in $r$ to fresh distinct constants. Then, $\mathcal{O} \models r$ iff $\mathcal{O} \cup\left\{\gamma_{i} \sigma\right\}_{i=1}^{n} \models \exists \mathbf{y} \psi \sigma$.

\subsection{Description Logics}

Description logics (DLs) (Baader et al., 2003) are a family of knowledge representation formalisms that correspond to decidable fragments of first-order logic. DLs are the logical formalisms underpinning the standard ontology languages: OWL DL is based on the description logic $\mathcal{S H O I N}$ (Horrocks, Patel-Schneider, \& van Harmelen, 2003), whereas

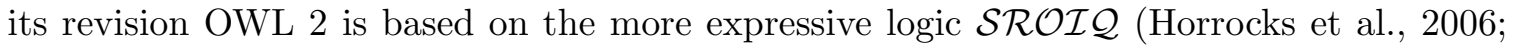
Cuenca Grau et al., 2008; W3C OWL Working Group, 2012). 
The basic building blocks in $\mathcal{S R O I Q}$ are pairwise disjoint countable sets of atomic concepts, which correspond to unary predicates, atomic roles, which correspond to binary predicates, and individuals, which correspond to constants. A role $R$ is either an atomic role or the inverse $S^{-}$of an atomic role $S$. Complex concepts are constructed according to the following grammar, where $\perp_{c}$ and $\top_{c}$ are the special bottom and top concepts, $A$ is an atomic concept, $R$ is a role, $o$ is an individual and $n \geq 1$ :

$$
\begin{aligned}
C::= & \perp_{c}\left|\top_{c}\right| A|\{o\}| \neg C\left|C_{1} \sqcap C_{2}\right| C_{1} \sqcup C_{2} \mid \\
& \exists R . C|\forall R . C| \exists R . \text { Self }|\geq n R . C| \leq n R . C
\end{aligned}
$$

We assume that concept expressions of the form $\geq 1 R$. $C$ are replaced by their equivalent $\exists$ R.C. A general concept inclusion axiom (GCI) is an expression of the form $C_{1} \sqsubseteq C_{2}$, where $C_{1}$ and $C_{2}$ are concepts. A role inclusion axiom (RIA) is an expression of the form $R_{1} \circ \cdots \circ R_{m} \sqsubseteq R$ where each $R_{i}$ is a role and $R$ is an atomic role. A role disjointness axiom is an expression of the form $\operatorname{Disj}\left(R_{1}, R_{2}\right)$ with $R_{1}$ and $R_{2}$ roles. Finally, a reflexivity axiom is an expression of the form $\operatorname{Ref}(R)$ with $R$ a role.

A $\mathcal{S R O I} \mathcal{Q}$ ontology is a finite set of GCIs, RIAs, role disjointness and reflexivity axioms. In order to ensure the decidability of basic reasoning tasks, each $\mathcal{S R O I Q}$ ontology must satisfy certain additional conditions (e.g., the set of RIAs must satisfy a regularity condition); these conditions are, however, immaterial to the results in this paper and we refer the reader to the work of Horrocks et al. (2006) for further details.

The semantics of $\mathcal{S R O I} \mathcal{Q}$ can be given by a direct translation into first-order logic (Baader et al., 2003; Motik, 2006) using the mapping function $\pi$ in Figure 1. Given a $\mathcal{S R O} \mathcal{I} \mathcal{Q}$ ontology $\mathcal{O}$, let $\mathcal{F}_{\mathcal{O}}=\{\pi(\alpha) \mid \alpha \in \mathcal{O}\}$; we then define

$$
\pi(\mathcal{O})=\mathcal{F}_{\mathcal{O}} \cup \mathcal{F}_{\mathcal{O}}^{\perp} \cup \mathcal{F}_{\mathcal{O}}^{\top} \cup \mathcal{F}_{\mathcal{O}}
$$

A first-order interpretation is a model of $\mathcal{O}$ if it is a model of $\pi(\mathcal{O})$.

Note that $\pi(\mathcal{O})$ is not always a set of first-order rules as defined in Section 2.1. However, $\mathcal{O}$ can always be polynomially normalised into an entailment preserving $\mathcal{S R O} \mathcal{I} \mathcal{Q}$ ontology $\mathcal{O}^{\prime}$ such that $\pi\left(\mathcal{O}^{\prime}\right)$ is a set of rules. We next define normalised $\mathcal{S} \mathcal{R O I} \mathcal{Q}$ ontologies and assume from here onwards (unless otherwise stated) that $\mathcal{S R O} \mathcal{I} \mathcal{Q}$ ontologies are normalised.

Definition 2. A $\mathcal{S R O I Q}$ ontology $\mathcal{O}$ is normalised if it consists only of axioms of the form

$$
\begin{gathered}
A \sqsubseteq \perp_{c} \quad A \sqsubseteq\{o\} \quad T_{c} \sqsubseteq A \quad\{o\} \sqsubseteq A \quad A \sqsubseteq B_{1} \sqcup B_{2} \quad A_{1} \sqcap A_{2} \sqsubseteq B \\
A \sqsubseteq \exists R . B \quad A \sqsubseteq \exists R . \text { Self } \quad \exists R . A \sqsubseteq B \quad \exists R . \text { Self } \sqsubseteq A \quad A \sqsubseteq \leq n R . B \\
R_{1} \circ R_{2} \sqsubseteq S \quad R^{-} \sqsubseteq S \quad \operatorname{Disj}(R, S) \quad \operatorname{Ref}(R)
\end{gathered}
$$

with $A_{(i)}, B_{(i)}$ atomic concepts, $o$ an individual, $R_{(i)}, S$ atomic roles, and $n \geq 1$.

Table 1 shows the application of $\pi$ to normalised axioms. Clearly, $\pi(\mathcal{O})$ is a set of rules whenever $\mathcal{O}$ is normalised. Moreover, $\pi$ establishes a bijection between $\mathcal{O}$ and $\pi(\mathcal{O})$ in this case. Since $\mathcal{O}$ and $\pi(\mathcal{O})$ are semantically equivalent, it is thus natural to identify them, and we shall do so in the remainder of this paper. 


\begin{tabular}{|l|l|}
\hline \multicolumn{1}{|c|}{$\alpha$} & \multicolumn{1}{c|}{$\pi(\alpha)$} \\
\hline$A \sqsubseteq \perp_{c}$ & $A(x) \rightarrow \perp$ \\
$A \sqsubseteq\{o\}$ & $A(x) \rightarrow x \approx o$ \\
$\top_{c} \sqsubseteq A$ & $\top(x) \rightarrow A(x)$ \\
$\{o\} \sqsubseteq A$ & $(\rightarrow A(o))$ \\
$A \sqsubseteq B_{1} \sqcup B_{2}$ & $A(x) \rightarrow B_{1}(x) \vee B_{2}(x)$ \\
$A_{1} \sqcap A_{2} \sqsubseteq B$ & $A_{1}(x) \wedge A_{2}(x) \rightarrow B(x)$ \\
$A \sqsubseteq \exists R . B$ & $A(x) \rightarrow \exists y[R(x, y) \wedge B(y)]$ \\
$A \sqsubseteq \exists R . S e l f$ & $A(x) \rightarrow R(x, x)$ \\
$\exists R . A \sqsubseteq B$ & $R(x, y) \wedge A(y) \rightarrow B(x)$ \\
$\exists R . \operatorname{Self} \sqsubseteq A$ & $R(x, x) \rightarrow A(x)$ \\
$A \sqsubseteq \leq n R . B$ & $A(x) \wedge \bigwedge_{i=1}^{n+1}\left[R\left(x, y_{i}\right) \wedge B\left(y_{i}\right)\right] \rightarrow \bigvee_{i \neq j} y_{i} \approx y_{j}$ \\
$R_{1} \circ R_{2} \sqsubseteq S$ & $R 1(x, y) \wedge R 2(y, z) \rightarrow S(x, z)$ \\
$R-\sqsubseteq S$ & $R(x, y) \rightarrow S(y, x)$ \\
$\operatorname{Disj}(R, S)$ & $R(x, y) \wedge S(x, y) \rightarrow \perp$ \\
$\operatorname{Ref}(R)$ & $\top(x) \rightarrow R(x, x)$ \\
\hline
\end{tabular}

Table 1: Correspondence between normalised $\mathcal{S R O} \mathcal{I} \mathcal{Q}$ axioms and rules.

Proposition 3. Let $\mathcal{O}$ be a $\mathcal{S R O I} \mathcal{Q}$ ontology and let $\mathcal{O}^{\prime}$ be the result of exhaustively applying to $\mathcal{O}$ the rewriting rules in Figure 2. Then, $\mathcal{O}^{\prime}$ satisfies the following properties: (i) it is normalised; (ii) it is of size polynomial in the size of $\mathcal{O}$ (assuming unary encoding of numbers); and (iii) it is a conservative extension of $\mathcal{O}$.

Proof. It is easy to see that a rewrite rule is always applicable to every axiom that is not normalised; furthermore, no rule is applicable to normalised axioms. Thus, $\mathcal{O}^{\prime}$ is normalised. Furthermore, note that the rules in Figure 2 are a syntactic variant of the structural transformation in first-order logic (Nonnengart \& Weidenbach, 2001). This implies that $\mathcal{O}^{\prime}$ can be computed in time polynomial in the size of $\mathcal{O}$ (assuming unary encoding of numbers), and also that it is a conservative extension of $\mathcal{O}$.

\subsection{Hyperresolution and Proofs}

Reasoning w.r.t. ontologies can be realised by means of hyperresolution (Robinson, 1965; Bachmair \& Ganzinger, 2001), which generalises forward chaining for datalog.

Hyperresolution is applicable to sets of first-order clauses - universally quantified sentences of the form $\bigwedge_{i} \gamma_{i} \rightarrow \bigvee_{j} \delta_{j}$ with $\gamma_{i}$ and $\delta_{j}$ atoms (possibly containing function symbols). Thus, it is only applicable to ontologies containing existentially quantified rules after Skolemisation and subsequent transformation into Conjunctive Normal Form (CNF).

For each rule $r$ of the form (1) and each existentially quantified variable $y$ in $r$, let $f_{y}^{r}$ be a function symbol globally unique for $r$ and $y$ of arity $|\mathbf{x}|$, and let $\theta_{\text {sk }}$ be the substitution such that $\theta_{\text {sk }}(y)=f_{y}^{r}(\mathbf{x})$ for each $r$ and $y$. The Skolemisation of $r$ is the sentence

$$
\mathrm{sk}(r)=\varphi(\mathbf{x}) \rightarrow \psi(\mathbf{x}, \mathbf{y}) \theta_{\text {sk }}
$$




$$
\begin{aligned}
& \perp_{c} \sqsubseteq C \Rightarrow \\
& D \sqsubseteq\{o\} \Rightarrow X \sqsubseteq\{o\}, D \sqsubseteq X \\
& \exists S^{-} . D \sqsubseteq C \Rightarrow \exists P . X \sqsubseteq D, S^{-} \sqsubseteq P \\
& \exists S . D \sqsubseteq C \Rightarrow \exists S . X \sqsubseteq C, D \sqsubseteq X \\
& \geq m S^{-} . D \sqsubseteq C \Rightarrow \geq m P \cdot D \sqsubseteq C, S^{-} \sqsubseteq P \\
& \geq m S . D \sqsubseteq C \Rightarrow \top_{c} \sqsubseteq \leq(m-1) S . D \sqcup C \\
& D \sqcap C_{1} \sqsubseteq C_{2} \Rightarrow X \sqcap C_{1} \sqsubseteq C_{2}, D \sqsubseteq X \\
& \neg C_{1} \sqsubseteq C_{2} \Rightarrow \top_{c} \sqsubseteq C_{1} \sqcup C_{2} \\
& C_{1} \sqcup C_{2} \sqsubseteq C_{3} \Rightarrow C_{1} \sqsubseteq C_{3}, C_{2} \sqsubseteq C_{3} \\
& \forall S^{-} . C_{1} \sqsubseteq C_{2} \Rightarrow \forall P . C_{1} \sqsubseteq C_{2}, P^{-} \sqsubseteq S \\
& \forall S . C_{1} \sqsubseteq C_{2} \Rightarrow \top_{c} \sqsubseteq \exists S . X \sqcup C_{2}, X \sqcap C \sqsubseteq \perp_{c} \\
& \exists S^{-} \text {.Self } \sqsubseteq C \Rightarrow \exists P \text {.Self } \sqsubseteq C, S^{-} \sqsubseteq P \\
& \leq(m-1) S^{-} . C_{1} \sqsubseteq C_{2} \Rightarrow \leq(m-1) P . C_{1} \sqsubseteq C_{2}, P^{-} \sqsubseteq S \\
& \leq(m-1) S \cdot C_{1} \sqsubseteq C_{2} \Rightarrow \top_{c} \sqsubseteq \geq m S . C_{1} \sqcup C_{2} \\
& C \sqsubseteq \top_{c} \Rightarrow \\
& \{o\} \sqsubseteq D \Rightarrow\{o\} \sqsubseteq X, X \sqsubseteq D \\
& C \sqsubseteq \exists S^{-} . D \Rightarrow C \sqsubseteq \exists P . D, P^{-} \sqsubseteq S \\
& C \sqsubseteq \exists S . D \Rightarrow C \sqsubseteq \exists S . X, X \sqsubseteq D \\
& C \sqsubseteq \forall S^{-} . D \Rightarrow C \sqsubseteq \forall P . D, S^{-} \sqsubseteq P \\
& C \sqsubseteq \forall S . D \quad \Rightarrow \quad C \sqsubseteq \forall S . X, X \sqsubseteq D \\
& C \sqsubseteq \exists S^{-} \text {.Self } \Rightarrow C \sqsubseteq \exists P \text {.Self, } P^{-} \sqsubseteq S \\
& C \sqsubseteq \leq n S^{-} . D \quad \Rightarrow \quad C \sqsubseteq \leq n P . D, S^{-} \sqsubseteq P \\
& C \sqsubseteq \leq n S . D \Rightarrow C \sqsubseteq \leq n S . X, X \sqsubseteq D \\
& C_{1} \sqsubseteq D \sqcup C_{2} \quad \Rightarrow \quad C_{1} \sqsubseteq X \sqcup C_{2}, X \sqsubseteq D \\
& C_{1} \sqsubseteq \neg C_{2} \Rightarrow C_{1} \sqcap C_{2} \sqsubseteq \perp_{c} \\
& C_{1} \sqsubseteq C_{2} \sqcap C_{3} \Rightarrow C_{1} \sqsubseteq C_{2}, C_{1} \sqsubseteq C_{3} \\
& C_{1} \sqsubseteq \geq m S^{-} . C_{2} \Rightarrow C_{1} \sqsubseteq \geq m P . C_{2}, P^{-} \sqsubseteq S \\
& C_{1} \sqsubseteq \geq m S . C_{2} \Rightarrow C_{1} \sqsubseteq \exists S . X_{i}, X_{i} \sqsubseteq C_{2}, X_{i} \sqcap X_{j} \sqsubseteq \perp_{c}(1 \leq i<j \leq m) \\
& D_{1} \sqsubseteq D_{2} \quad \Rightarrow \quad D_{1} \sqsubseteq X, X \sqsubseteq D_{2} \\
& R_{1} \circ R_{2} \circ R_{3} \circ \cdots \circ R_{k} \sqsubseteq S \Rightarrow R_{1} \circ R_{2} \sqsubseteq P, P \circ R_{3} \circ \cdots \circ R_{k} \sqsubseteq S \\
& Q^{-} \circ R \sqsubseteq S \Rightarrow P \circ R \sqsubseteq S, Q^{-} \sqsubseteq P \\
& R \circ Q^{-} \sqsubseteq S \Rightarrow R \circ P \sqsubseteq S, Q^{-} \sqsubseteq P \\
& \operatorname{Disj}\left(R, Q^{-}\right) \Rightarrow \operatorname{Disj}(R, P), Q^{-} \sqsubseteq P \\
& \operatorname{Disj}\left(Q^{-}, R\right) \Rightarrow \operatorname{Disj}(P, R), Q^{-} \sqsubseteq P \\
& \operatorname{Ref}\left(Q^{-}\right) \Rightarrow \operatorname{Ref}(Q)
\end{aligned}
$$

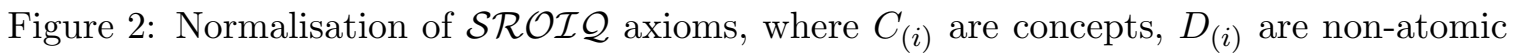
concepts different from $\perp_{c}$ and $\top_{c}, X$ is a fresh atomic concept, $Q$ and $S$ are atomic roles, $R_{(i)}$ are roles, $P$ is a fresh atomic role, and $m \geq 2, n \geq 1, k \geq 3$.

A CNF of $\operatorname{sk}(r)$ is a set of first-order clauses that is a conservative extension of sk $(r)$. Such a CNF can be obtained in polynomial time using the standard structural transformation (Nonnengart \& Weidenbach, 2001). In this paper we consider an arbitrary but fixed function 
$\kappa$ mapping each rule $r$ to some CNF of sk $(r)$. The function $\kappa$ extends to ontologies in the obvious way, and we refer to $\kappa(\mathcal{O})$ as a clausification of $\mathcal{O}$. By the well-known properties of Skolemisation and the structural transformation we have that $\mathcal{O} \models \phi$ iff $\kappa(\mathcal{O}) \models \phi$ for each ontology $\mathcal{O}$ and each first-order sentence $\phi$ over $\operatorname{Sig}(\mathcal{O})$.

Let $r=\bigwedge_{i=1}^{n} \gamma_{i} \rightarrow \bigvee_{j=1}^{m} \delta_{j}$ be a clause and let $\varphi_{i}=\psi_{i} \vee \xi_{i}$ with $1 \leq i \leq n$ be ground disjunctions of atoms where $\xi_{i}$ is a single atom; furthermore, let $\sigma$ be a most general unifier (MGU) of each $\gamma_{i}, \xi_{i}$. The ground disjunction of atoms $\bigvee_{i=1}^{n} \psi_{i} \bigvee \bigvee_{j=1}^{m} \delta_{j} \sigma$ is a hyperresolvent of $r$ and $\varphi_{1}, \ldots, \varphi_{n}$. This disjunction can be empty, in which case we denote it with $\square$. Let $\mathcal{C}$ be a set of clauses, $\mathcal{D}$ a dataset and $\varphi$ a disjunction of ground atoms. A hyperresolution proof (or simply a proof) of $\varphi$ in $\mathcal{C} \cup \mathcal{D}$ is a pair $\rho=(T, \lambda)$ where $T$ is a directed, rooted tree, and $\lambda$ is a mapping from nodes in $T$ to disjunctions of ground atoms such that for each node $v$ in $T$ the following properties are satisfied:

1. if $v$ is the root of $T$ then $\lambda(v)=\varphi$,

2. if $v$ is a leaf in $T$ then either $(\rightarrow \lambda(v)) \in \mathcal{C}$ or $\lambda(v) \in \mathcal{D}$, and

3. if $v$ has children $w_{1}, \ldots, w_{n}$ then $\lambda(v)$ is a hyperresolvent of a clause from $\mathcal{C}$ and $\lambda\left(w_{1}\right), \ldots, \lambda\left(w_{n}\right)$.

The support of $\rho$, denoted by $\operatorname{supp}(\rho)$, is the set of clauses in $\mathcal{C}$ that take part in $\rho$ as described in properties 2 and 3 above. We write $\mathcal{C} \cup \mathcal{D} \vdash \varphi$ to indicate that there exists a proof of $\varphi$ in $\mathcal{C} \cup \mathcal{D}$. Hyperresolution is sound (if $\mathcal{C} \cup \mathcal{D} \vdash \varphi$ then $\mathcal{C} \cup \mathcal{D} \models \varphi$ ), and complete in the following sense: if $\mathcal{C} \cup \mathcal{D} \models \varphi$ then there exists $\psi \subseteq \varphi$ such that $\mathcal{C} \cup \mathcal{D} \vdash \psi$ (Robinson, 1965). In particular, $\mathcal{C}$ is unsatisfiable iff $\mathcal{C} \cup \mathcal{D} \vdash \square$.

Given proofs $\rho=(T, \lambda)$ and $\rho^{\prime}=\left(T^{\prime}, \lambda^{\prime}\right)$, we say that $\rho$ is embeddable into $\rho^{\prime}$ if there exists a mapping $\iota: T \rightarrow T^{\prime}$ satisfying the following properties for each $v \in T:$ (i) if $v$ is a leaf of $T$, then $\iota(v)$ is a leaf of $T^{\prime}$; (ii) if $w$ is an ancestor of $v$ in $T$ then $\iota(w)$ is an ancestor of $\iota(v)$ in $T^{\prime}$, and (iii) $\lambda(v) \subseteq \lambda^{\prime}(\iota(v)) \cup\{\perp\}$. Furthermore, given a substitution $\tau$, we say that $\rho$ is embeddable into $\rho^{\prime}$ modulo $\tau$ if it is embeddable into the proof $\left(T^{\prime}, \lambda_{\tau}^{\prime}\right)$, where $\lambda_{\tau}^{\prime}(v)=\lambda^{\prime}(v) \tau$ for each $v \in T^{\prime}$.

\section{Module Extraction}

In this section, we recapitulate the key notions of inseparability relation and module that have been proposed in the description logic literature (Cuenca Grau et al., 2008; Kontchakov et al., 2010; Konev et al., 2013; Sattler et al., 2009; Konev et al., 2009). Furthermore, when required, we adapt these notions to the setting of first-order rules and prove some basic results that will be exploited throughout this paper.

\subsection{Inseparability Relations and Modules}

Intuitively, given an ontology $\mathcal{O}$ and a signature $\Sigma$, a module of $\mathcal{O}$ w.r.t. $\Sigma$ is a subset $\mathcal{M}$ of $\mathcal{O}$ that is indistinguishable from $\mathcal{O}$ w.r.t. reasoning tasks where only predicates in $\Sigma$ are considered of interest. The indistinguishability criteria depend on the specific task at hand, and are usually formalised by means of inseparability relations. 
Definition 4. An inseparability relation is a family $\mathcal{S}=\left\{\equiv_{\Sigma}^{\mathcal{S}} \mid \Sigma\right.$ a set of predicates $\}$ of equivalence relations between ontologies satisfying the following properties:

- if $\mathcal{O}^{\prime}$ is a conservative extension of $\mathcal{O}$ and $\Sigma=\operatorname{Sig}(\mathcal{O})$, then $\mathcal{O} \equiv{ }_{\Sigma}^{\mathcal{S}} \mathcal{O}^{\prime}$; and

- $\mathcal{O}_{1} \equiv_{\Sigma}^{\mathcal{S}} \mathcal{O}$ implies $\mathcal{O}_{2} \equiv_{\Sigma}^{\mathcal{S}} \mathcal{O}$ for all $\mathcal{O}_{1} \subseteq \mathcal{O}_{2} \subseteq \mathcal{O}$ and $\Sigma$.

The first property ensures that inseparability is stable under model-preserving transformations, whereas the second one ensures that it is consistent with the monotonicity of first-order logic. The following definition captures the most common inseparability relations studied in the literature.

Definition 5. For a signature $\Sigma$, we say that ontologies $\mathcal{O}$ and $\mathcal{O}^{\prime}$ are

- $\Sigma$-model inseparable $\left(\mathcal{O} \equiv \sum_{\Sigma}^{m} \mathcal{O}^{\prime}\right)$, if for every model $\mathcal{I}$ of $\mathcal{O}$ (resp. of $\left.\mathcal{O}^{\prime}\right)$ there exists a model $\mathcal{J}$ of $\mathcal{O}^{\prime}$ (resp. of $\mathcal{O}$ ) with the same domain such that $A^{\mathcal{I}}=A^{\mathcal{J}}$ for each $A \in \Sigma$.

- $\Sigma$-query inseparable $\left(\mathcal{O} \equiv_{\Sigma}^{\mathrm{q}} \mathcal{O}^{\prime}\right)$ if for each Boolean PEQ $q$ over $\Sigma$ and dataset $\mathcal{D}$ over $\Sigma$ we have $\mathcal{O} \cup \mathcal{D}=q$ iff $\mathcal{O}^{\prime} \cup \mathcal{D} \models q$.

- $\Sigma$-fact inseparable $\left(\mathcal{O} \equiv_{\Sigma}^{\mathrm{f}} \mathcal{O}^{\prime}\right)$ if for each fact $\gamma$ over $\Sigma$ and dataset $\mathcal{D}$ over $\Sigma$ we have $\mathcal{O} \cup \mathcal{D} \models \gamma$ iff $\mathcal{O}^{\prime} \cup \mathcal{D} \models \gamma$.

- $\Sigma$-implication inseparable $\left(\mathcal{O} \equiv_{\Sigma}^{\mathrm{i}} \mathcal{O}^{\prime}\right)$ if for each $\Sigma$-rule $r$ of the form $A(\mathbf{x}) \rightarrow B(\mathbf{x})$ we have $\mathcal{O} \models r$ iff $\mathcal{O}^{\prime}=r$.

A few observations about the notions introduced in Definition 5 are in order. First, note that in the definition of query and fact inseparability the quantification over queries and datasets is relative to the same signature $\Sigma$; this is the standard convention adopted in the literature (Lutz \& Wolter, 2010; Baader, Bienvenu, Lutz, \& Wolter, 2010). Second, the restriction to Boolean queries in the definition of query inseparability is strictly technical: the obvious extension to non-Boolean queries leads to an equivalent definition. Finally, observe that our notion of fact inseparability is the natural generalisation of inseparability w.r.t. atomic instance queries in description logics (Lutz \& Wolter, 2010).

Example 6. Let us consider the ontology $\mathcal{O}^{e x}$ from Figure 3, which will serve as a running example. Let us also consider the signatures $\Sigma_{i}$ and fragments $\mathcal{M}_{i}$ of $\mathcal{O}^{e x}$ given next:

$$
\begin{array}{ll}
\Sigma_{1}=\{B, C, D, H\} & \mathcal{M}_{1}=\left\{r_{5}, r_{6}, r_{7}, r_{8}\right\} \\
\Sigma_{2}=\{A, B\} & \mathcal{M}_{2}=\emptyset \\
\Sigma_{3}=\{A, C, D, R\} & \mathcal{M}_{3}=\left\{r_{1}, r_{2}\right\}
\end{array}
$$

The only non-tautological $\Sigma_{1}$-implication entailed by $\mathcal{O}^{e x}$ is $D(x) \rightarrow H(x)$, which also follows from $\mathcal{M}_{1}$; thus, $\mathcal{M}_{1}$ is $\Sigma_{1}$-implication inseparable from $\mathcal{O}^{e x}$. Furthermore, any subset of $\mathcal{O}^{e x}$ not containing $\mathcal{M}_{1}$ does not entail $D(x) \rightarrow H(x)$ and is hence not $\Sigma_{1}$-implication inseparable from $\mathcal{O}^{e x}$. As we will see later on, the requirement of fact inseparability is stronger than that of implication inseparability; indeed, $\mathcal{M}_{1}$ is not $\Sigma_{1}$-fact inseparable from $\mathcal{O}^{e x}$ since, for $\mathcal{D}_{1}=\{B(a), C(a)\}$, we have $\mathcal{O}^{e x} \cup \mathcal{D}_{1} \models D(a)$ but $\mathcal{M}_{1} \cup \mathcal{D}_{1} \not \models D(a)$. 


$$
\begin{array}{rl|c}
r_{1}: & A(x) \rightarrow \exists y_{1}\left[R\left(x, y_{1}\right) \wedge B\left(y_{1}\right)\right] & A \sqsubseteq \exists R . B \\
r_{2}: & A(x) \rightarrow R(x, o) & A \sqsubseteq \exists R .\{o\} \\
r_{3}: & B(x) \wedge C(x) \rightarrow D(x) & B \sqcap C \sqsubseteq D \\
r_{4}: & R(x, y) \wedge C(y) \rightarrow E(x) & \exists R . C \sqsubseteq E \\
r_{5}: & D(x) \rightarrow F(x) \vee G(x) & D \sqsubseteq F \sqcup G \\
r_{6}: & F(x) \rightarrow \exists y_{2} S\left(x, y_{2}\right) & F \sqsubseteq \exists S . \top_{c} \\
r_{7}: & S(x, y) \rightarrow H(x) & \exists S . \top_{c} \sqsubseteq H \\
r_{8}: & G(x) \rightarrow H(x) & G \sqsubseteq H
\end{array}
$$

Figure 3: Example ontology $\mathcal{O}^{e x}$ in both rule and DL notation.

It can be checked that $\mathcal{M}_{2}$ is $\Sigma_{2}$-fact inseparable from $\mathcal{O}^{e x}$. It is, however, not $\Sigma_{2}$-query inseparable: for $\mathcal{D}_{2}=\{A(a)\}$, we have $\mathcal{O}^{e x} \cup \mathcal{D}_{2} \mid=\exists y B(y)$ but $\mathcal{M}_{2} \cup \mathcal{D}_{2} \not \models \exists y B(y)$.

Finally, consider $\mathcal{M}_{3}$ and $\Sigma_{3}$. As we will see later on, $\mathcal{M}_{3}$ is $\Sigma_{3}$-query inseparable from $\mathcal{O}^{e x}$; however, it is not $\Sigma_{3}$-model inseparable. Indeed, the interpretation $\mathcal{I}$ where $\Delta^{\mathcal{I}}=\{a, o\}, A^{\mathcal{I}}=\{a\}, B^{\mathcal{I}}=C^{\mathcal{I}}=\{o\}, D^{\mathcal{I}}=\emptyset$ and $R^{\mathcal{I}}=\{(a, o)\}$ is a model of $\mathcal{M}_{3}$. This interpretation, however, cannot be extended to a model of $r_{3}$ (or, consequently, to a model of $\mathcal{O}$ ) without reinterpreting $A, C, D$ or $R$. We will also see that to ensure $\Sigma_{3}$-model inseparability it suffices to extend $\mathcal{M}_{3}$ with rule $r_{3}$.

Model inseparability can be characterised in terms of preservation of second-order consequences (Konev et al., 2013): ontologies $\mathcal{O}$ and $\mathcal{O}^{\prime}$ are $\Sigma$-model inseparable if and only if for each second-order $\Sigma$-sentence $\varphi$ we have $\mathcal{O} \models \varphi$ iff $\mathcal{O}^{\prime} \models \varphi$. Additionally, as we show next, query and fact inseparability can be characterised in terms of preservation of first-order rules and datalog rules, respectively.

Proposition 7. The following statements hold for each signature $\Sigma$ and each pair of ontologies $\mathcal{O}_{1}$ and $\mathcal{O}_{2}$ :

1. $\mathcal{O}_{1} \equiv_{\Sigma}^{\mathrm{q}} \mathcal{O}_{2}$ iff $\mathcal{O}_{1}=r \Leftrightarrow \mathcal{O}_{2}=r$ holds for each $\Sigma$-rule $r$ with non-empty head.

2. $\mathcal{O}_{1} \equiv_{\Sigma}^{f} \mathcal{O}_{2}$ iff $\mathcal{O}_{1}=r \Leftrightarrow \mathcal{O}_{2} \models r$ holds for each datalog $\Sigma$-rule $r$ with non-empty head.

Proof. We prove the first statement; the second one is analogous. Suppose $\mathcal{O}_{1} \equiv_{\Sigma}^{q} \mathcal{O}_{2}$ and consider an arbitrary rule $r=\bigwedge_{i=1}^{n} \gamma_{i}(\mathbf{x}) \rightarrow \exists \mathbf{y} \psi(\mathbf{x}, \mathbf{y})$ over $\Sigma$ such that $\psi \neq \square$, and a substitution $\sigma$ mapping universally quantified variables in $r$ to fresh distinct constants. Furthermore, consider the dataset $\mathcal{D}=\left\{\gamma_{i} \sigma\right\}_{i=1}^{n}$, and the Boolean PEQ $q=\exists \mathbf{y} \psi(\mathbf{x}, \mathbf{y}) \sigma$ (note that $q$ is indeed a Boolean PEQ since by hypothesis $\psi$ is non-empty). By Proposition 1, $\mathcal{O}_{i}=r$ iff $\mathcal{O}_{i} \cup \mathcal{D} \mid=q$. Together with $\mathcal{O}_{1} \equiv_{\Sigma}^{q} \mathcal{O}_{2}$, this implies that $\mathcal{O}_{1}=r \Leftrightarrow \mathcal{O}_{2}=r$.

Assume now that $\mathcal{O}_{1} \models r \Leftrightarrow \mathcal{O}_{2} \models r$ holds for each $\Sigma$-rule $r$ with a non-empty head. Let $q$ be a Boolean PEQ over $\Sigma$ and $\mathcal{D}$ a $\Sigma$-dataset and consider the rule $r=\bigwedge_{\gamma \in \mathcal{D}} \gamma \rightarrow q$. By Proposition 1 we have $\mathcal{O}_{i} \cup \mathcal{D} \models q$ iff $\mathcal{O}_{i}=r$, and since, by assumption, $\mathcal{O}_{1} \models r \Leftrightarrow \mathcal{O}_{2} \models r$, it follows that $\mathcal{O}_{1} \cup \mathcal{D}=q \Leftrightarrow \mathcal{O}_{2} \cup \mathcal{D}=q$ and hence $\mathcal{O}_{1}$ and $\mathcal{O}_{2}$ are $\Sigma$-query inseparable.

It immediately follows that the inseparability relations in Definition 5 are naturally ordered from strongest to weakest for each non-trivial $\Sigma$ as given next:

$$
\equiv_{\Sigma}^{\mathrm{m}} \subsetneq \equiv_{\Sigma}^{\mathrm{q}} \subsetneq \equiv_{\Sigma}^{\mathrm{f}} \subsetneq \equiv_{\Sigma}^{\mathrm{i}}
$$


Furthermore, we can now identify the classes of entailments that are relevant to each inseparability relation.

Definition 8. For each inseparability relation $\mathcal{S} \in\{\mathrm{m}, \mathrm{q}, \mathrm{f}, \mathrm{i}\}$, let rel $\mathcal{S}$ be the function mapping each ontology $\mathcal{O}$ and signature $\Sigma$ to a set of relevant entailments as follows: $\operatorname{rel}_{\mathcal{S}}(\mathcal{O}, \Sigma)= \begin{cases}\{\phi \mid \mathcal{O} \models \phi \text { and } \phi \text { is a second-order } \Sigma \text {-sentence }\} & \text { if } \mathcal{S}=\mathrm{m} \\ \{r \mid \mathcal{O} \models r \text { and } r \text { is a } \Sigma \text {-rule with non-empty head }\} & \text { if } \mathcal{S}=\mathrm{q} \\ \{r \mid \mathcal{O} \models r \text { and } r \text { is a datalog } \Sigma \text {-rule with non-empty head }\} & \text { if } \mathcal{S}=\mathrm{f} \\ \{r \mid \mathcal{O} \models r \text { and } r \text { is of the form } A(\mathbf{x}) \rightarrow B(\mathbf{x}) \text { with } A, B \in \Sigma\} & \text { if } \mathcal{S}=\mathrm{i}\end{cases}$

The following theorem establishes that the inseparability relations in Definition 5 are fully characterised by the preservation of relevant $\Sigma$-entailments as in Definition 8 .

Theorem 9. Let $\mathcal{O}$ and $\mathcal{O}^{\prime}$ be ontologies, $\Sigma$ a signature, and let $\mathcal{S} \in\{\mathrm{m}, \mathrm{q}, \mathrm{f}, \mathrm{i}\}$. Then, $\mathcal{O} \equiv \mathcal{S}_{\Sigma} \mathcal{O}^{\prime}$ if and only if $\operatorname{rel}_{\mathcal{S}}(\mathcal{O}, \Sigma)=\operatorname{rel}_{\mathcal{S}}\left(\mathcal{O}^{\prime}, \Sigma\right)$.

Proof. A direct consequence of Definitions 5 and 8, Proposition 7 and the characterisation of model inseparability in terms of second-order entailments in (Konev et al., 2013).

Inseparability relations allow us to formalise modules as well as their desirable properties.

Definition 10. Let $\mathcal{O}$ be an ontology, $\Sigma$ a signature, and $\mathcal{S}$ an inseparability relation, and let $\mathcal{M} \subseteq \mathcal{O}$. We say that $\mathcal{M}$ is a $\equiv_{\Sigma}^{\mathcal{S}}$-module of $\mathcal{O}$ if $\mathcal{O} \equiv \equiv_{\Sigma}^{\mathcal{S}} \mathcal{M}$. Furthermore, $\mathcal{M}$ is

- minimal if no $\mathcal{M}^{\prime} \subsetneq \mathcal{M}$ is a $\equiv{ }_{\Sigma}^{\mathcal{S}}$-module of $\mathcal{O}$;

- self-contained if $\mathcal{O} \equiv{ }_{\Sigma \cup \operatorname{Sig}(\mathcal{M})}^{\mathcal{M}} \mathcal{M}$;

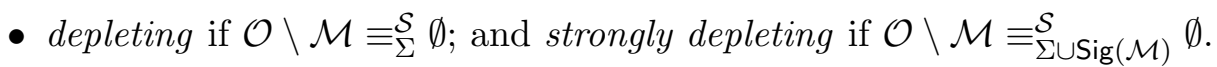

Finally, we define a justification in $\mathcal{O}$ of a sentence $\phi$ such that $\mathcal{O}=\phi$ as a subset-minimal $\mathcal{O}^{\prime} \subseteq \mathcal{O}$ such that $\mathcal{O}^{\prime}=\phi$. We say that $\mathcal{M}$ is justification-preserving if for each $\phi$ in $\operatorname{rel}_{\mathcal{S}}(\mathcal{O}, \Sigma)$ and each justification $\mathcal{O}^{\prime}$ of $\phi$ in $\mathcal{O}$ we have $\mathcal{O}^{\prime} \subseteq \mathcal{M}$.

Example 11. Consider again the ontologies and signatures in Example 6. We can see that each $\mathcal{M}_{i}$ is a module of $\mathcal{O}^{e x}$; in particular, $\mathcal{M}_{1}$ is a $\equiv_{\Sigma_{1}}^{i}$-module, $\mathcal{M}_{2}$ is a $\equiv_{\Sigma_{2}}^{f}$-module, $\mathcal{M}_{3}$ is a $\equiv_{\Sigma_{3}}^{\mathrm{q}}$-module, and $\mathcal{M}_{3} \cup\left\{r_{3}\right\}$ is a $\equiv_{\Sigma_{3}}^{\mathrm{m}}$-module.

The inseparability requirement ensures that modules can be used instead of $\mathcal{O}$ for reasoning purposes, provided that the entailments relevant to the application at hand are captured by the given inseparability relation and contain only symbols from $\Sigma$.

Minimality ensures that the module contains as little irrelevant information as possible while still satisfying the inseparability requirement. Although minimality is clearly desirable in most applications of modules (e.g., reasoning over small ontology subsets is typically preferable to reasoning over the whole ontology), extracting modules of minimal size is invariably very hard (and often algorithmically infeasible) (Lutz \& Wolter, 2010; Konev 
et al., 2013); thus, practical techniques aim at computing modules that are typically much smaller than $\mathcal{O}$, albeit not necessarily minimal.

Self-contained modules are inseparable from $\mathcal{O}$ not only w.r.t. the relevant signature $\Sigma$, but also w.r.t. their own signature. Depletingness ensures that no relevant information is "left behind" after extracting the module from $\mathcal{O}$, i.e., that $\mathcal{O} \backslash \mathcal{M}$ is inseparable from the empty ontology. The most basic form of depletingness is formulated in terms of $\Sigma$, whereas a stronger variant requires inseparability w.r.t. the symbols in $\mathcal{M}$ as well. Selfcontained and depleting modules are especially well-suited for ontology reuse and modular ontology development applications. For instance, if $\mathcal{M}$ is both self-contained and depleting, the developer of $\mathcal{O}$ can remodel the sub-domain characterised by $\Sigma$ by replacing $\mathcal{M}$ in $\mathcal{O}$ with a new set of axioms, with the guarantee that any changes performed will not have any unintended interactions with the rest of $\mathcal{O}$.

Justification-preservation enables the use of modules for ontology debugging and repair (Schlobach \& Cornet, 2003; Kalyanpur et al., 2007; Kalyanpur, Parsia, Sirin, \& Hendler, 2005; Horridge, Parsia, \& Sattler, 2008; Kalyanpur, Parsia, Sirin, \& Cuenca Grau, 2006). The justification of an entailment is a useful form of explanation; furthermore, ontology repair services typically rely on the computation of all justifications of an unintended entailment as a first step towards obtaining a repair plan. Computing justifications, however, is a computationally intensive task and practical module extraction techniques have been effectively exploited to optimise this process (Suntisrivaraporn et al., 2008).

We conclude this section by briefly discussing the impact of normalisation on module extraction. As pointed out in Section 2.2, our technical results are applicable to ontologies consisting of rules; when referring to DL ontologies, we implicitly assume they are given in rule form and are therefore normalised. We argue that normalisation techniques stemming from the structural transformation preserve inseparability and hence it is possible to obtain a module for a DL ontology once a module for its normalisation has been computed.

Definition 12. A normalisation function norm maps $\mathcal{S} \mathcal{R O} \mathcal{I} \mathcal{Q}$ ontologies to normalised $\mathcal{S R O I} \mathcal{Q}$ ontologies s.t. the following holds for all ontologies in its domain:

- $\operatorname{norm}(\mathcal{O})$ is a conservative extension of $\mathcal{O}$; and

- $\mathcal{O}_{1} \subseteq \mathcal{O}_{2}$ implies norm $\left(\mathcal{O}_{1}\right) \subseteq \operatorname{norm}\left(\mathcal{O}_{2}\right)$.

Definition 12 captures the standard normalisation techniques stemming from the structural transformation, such as the one discussed in Section 2.2. Furthermore, it is typically straightforward in practice to keep track of the correspondence between the axioms in the original ontology $\mathcal{O}$ and those in norm $(\mathcal{O})$. As shown by the following proposition, this correspondence allows us to efficiently obtain a module of $\mathcal{O}$ once a module for norm $(\mathcal{O})$ has been computed.

Proposition 13. Let $\Sigma$ be a signature, $\mathcal{S}$ an inseparability relation, and norm a normalisation function. Then, $\mathcal{M} \equiv_{\Sigma}^{\mathcal{S}} \mathcal{O}$ iff $\operatorname{norm}(\mathcal{M}) \equiv_{\Sigma}^{\mathcal{S}} \operatorname{norm}(\mathcal{O})$.

Proof. By definition, $\mathcal{S}$ satisfies that $\mathcal{O}_{1} \subseteq \mathcal{O}_{2} \subseteq \mathcal{O}$ and $\mathcal{O}_{1} \equiv_{\Sigma}^{\mathcal{S}} \mathcal{O}$ implies $\mathcal{O}_{2} \equiv \mathcal{S} \mathcal{O}$. Therefore, if norm $(\mathcal{M})$ contains a $\equiv_{\Sigma}^{\mathcal{S}}$-module of $\operatorname{norm}(\mathcal{O})$ then norm $(\mathcal{M})$ is a $\equiv_{\Sigma}^{\mathcal{S}}$-module of norm $(\mathcal{O})$ itself. On the other hand, since norm $(\mathcal{O})$ (resp. norm $(\mathcal{M})$ ) is a conservative 


\begin{tabular}{|c|l|l|}
\hline$\alpha$ & $\perp$-local w.r.t. $\Sigma$ & T-local w.r.t. $\Sigma$ \\
\hline$A \sqsubseteq \perp_{c}$ & if $A \notin \Sigma$ & never \\
$A \sqsubseteq\{o\}$ & if $A \notin \Sigma$ & never \\
$\top_{c} \sqsubseteq A$ & never & if $A \notin \Sigma$ \\
$\{o\} \sqsubseteq A$ & never & if $A \notin \Sigma$ \\
$A \sqsubseteq B_{1} \sqcup B_{2}$ & if $A \notin \Sigma$ & if $B_{1} \notin \Sigma$ or $B_{2} \notin \Sigma$ \\
$A_{1} \sqcap A_{2} \sqsubseteq B$ & if $A_{1} \notin \Sigma$ or $A_{2} \notin \Sigma$ & if $B \notin \Sigma$ \\
$A \sqsubseteq \exists R . B$ & if $A \notin \Sigma$ & if $\{R, B\} \cap \Sigma=\emptyset$ \\
$A \sqsubseteq \exists R . S e l f$ & if $A \notin \Sigma$ & if $R \notin \Sigma$ \\
$\exists R . A \sqsubseteq B$ & if $R \notin \Sigma$ or $A \notin \Sigma$ & if $B \notin \Sigma$ \\
$\exists R . S e l f \sqsubseteq A$ & if $R \notin \Sigma$ & if $B \notin \Sigma$ \\
$A \sqsubseteq \leq m R . B$ & if $A \notin \Sigma$ or $R \notin \Sigma$ or $B \notin \Sigma$ & never \\
$R_{1} \circ R_{2} \sqsubseteq S$ & if $R_{1} \notin \Sigma$ or $R R_{2} \notin \Sigma$ & if $S \notin \Sigma$ \\
$R \sqsubseteq S-$ & if $R \notin \Sigma$ & if $S \notin \Sigma$ \\
$\operatorname{Disj}(R, S)$ & if $R \notin \Sigma$ or $S \notin \Sigma$ & never \\
$\operatorname{Ref}(R)$ & never & if $R \notin \Sigma$ \\
\hline
\end{tabular}

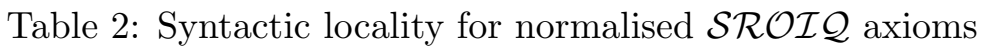

extension of $\mathcal{O}$ (resp. of $\mathcal{M}$ ), and we can asume w.l.o.g. that $\Sigma$ contains no symbols from $\operatorname{Sig}(\operatorname{norm}(\mathcal{O})) \backslash \operatorname{Sig}(\mathcal{O})$, we have that $\operatorname{norm}(\mathcal{O}) \equiv_{\Sigma}^{\mathcal{S}} \mathcal{O}\left(\operatorname{resp}\right.$. norm $\left.(\mathcal{M}) \equiv_{\Sigma}^{\mathcal{S}} \mathcal{M}\right)$. Since $\equiv_{\Sigma}^{\mathcal{S}}$ is an equivalence relation, it follows that $\mathcal{M} \equiv{ }_{\Sigma}^{\mathcal{S}} \mathcal{O}$ iff $\operatorname{norm}(\mathcal{M}) \equiv_{\Sigma}^{\mathcal{S}} \operatorname{norm}(\mathcal{O})$.

\subsection{Syntactic Locality}

For many of the inseparability relations introduced in Section 3.1, checking whether $\mathcal{M}$ is a module for $\mathcal{O}$ w.r.t. $\Sigma$ is typically of very high complexity, and often undecidable, even for rather lightweight ontology languages (Lutz \& Wolter, 2010; Konev et al., 2013).

Consequently, practical module extraction techniques are typically based on approximations, which ensure that the computed $\mathcal{M}$ is a module, yet not necessarily a minimal one. One such approximation that is often exploited in practice is based on the notion of syntactic locality (Cuenca Grau et al., 2007a; Cuenca Grau, Horrocks, Kazakov, \& Sattler, 2007b; Sattler et al., 2009; Cuenca Grau et al., 2008).

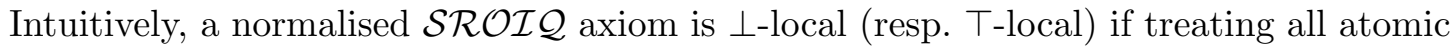
concepts and roles outside $\Sigma$ as the $\perp$ (resp. $\top$ ) concept and role, respectively, leads to the axiom being an "obvious" tautology.

Definition 14. A normalised $\mathcal{S R O \mathcal { I } Q}$ axiom $\alpha$ is $\perp$-local (resp. T-local) w.r.t. a signature $\Sigma$ if it satisfies the conditions given in the second (resp. third) column in Table 2. A normalised $\mathcal{S R O I} \mathcal{Q}$ ontology $\mathcal{O}$ is $\perp$-local (resp. $T$-local) w.r.t. $\Sigma$ if each of its axioms is $\perp$-local (resp. $T$-local) w.r.t. $\Sigma$. Finally, we say that $\mathcal{O}$ is local w.r.t. $\Sigma$ if it is either $\perp$-local or $\top$-local w.r.t. $\Sigma$.

The key properties of $\perp$ - and $T$-locality, established in the existing literature (Cuenca Grau et al., 2008; Sattler et al., 2009), are summarised in the following proposition. 
Proposition 15. Let $\mathcal{O}$ be a $\mathcal{S R O I} \mathcal{Q}$ ontology, $\Sigma$ a signature and $x \in\{\perp, \top\}$.

1. If $\mathcal{O}$ is $x$-local w.r.t. $\Sigma$ then $\mathcal{O} \equiv \equiv_{\Sigma}^{m} \emptyset$.

2. If $\mathcal{M} \subseteq \mathcal{O}$ and $\mathcal{O} \backslash \mathcal{M}$ is x-local w.r.t. $\Sigma \cup \operatorname{Sig}(\mathcal{M})$, then $\mathcal{M}$ is a self-contained, strongly depleting, and justification-preserving $\equiv_{\Sigma}^{\mathrm{m}}$-module of $\mathcal{O}$.

Property 2 in Proposition 15 immediately suggests the notion of locality-based module.

Definition 16. Let $\mathcal{O}$ be a normalised $\mathcal{S R O I} \mathcal{Q}$ ontology, $\Sigma$ a signature, and $x \in\{\perp, \top\}$. The $x$-module for $\mathcal{O}$ w.r.t. $\Sigma$, denoted $\mathcal{M}_{[\mathcal{O}, \Sigma]}^{x}$, is the smallest subset $\mathcal{M} \subseteq \mathcal{O}$ such that $\mathcal{O} \backslash \mathcal{M}$ is $x$-local w.r.t. $\Sigma \cup \operatorname{Sig}(\mathcal{M})$.

The $\perp T^{*}$-module for $\mathcal{O}$ w.r.t. $\Sigma$ is the least fixpoint of the sequence $\left\{\mathcal{M}_{i}\right\}_{i \geq 1}$ where $\mathcal{M}_{1}=\mathcal{M}_{[\mathcal{O}, \Sigma]}^{\perp}$ and $\mathcal{M}_{i}$ is defined as follows for $i \geq 2$ :

$$
\mathcal{M}_{i}= \begin{cases}\mathcal{M}_{\left[\mathcal{M}_{i-1}, \Sigma\right]}^{\top} & \text { if } i \text { is odd } \\ \mathcal{M}_{\left[\mathcal{M}_{i-1}, \Sigma\right]}^{\perp} & \text { if } i \text { is even }\end{cases}
$$

Example 17. Consider ontology $\mathcal{O}^{e x}$ from Figure 3 and the signature $\Sigma=\{B, C, D, R\}$. We have that $\mathcal{M}_{\left[\mathcal{O}^{e x}, \Sigma\right]}^{\perp}=\left\{r_{3}-r_{8}\right\}, \mathcal{M}_{\left[\mathcal{O}^{e x}, \Sigma\right]}^{\top}=\left\{r_{1}-r_{3}\right\}$, and $\mathcal{M}_{\left[\mathcal{O}^{e x}, \Sigma\right]}^{\perp \top^{*}}=\left\{r_{3}\right\}$.

Locality-based modules as in Definition 16 can be computed in polynomial time. Furthermore, by Proposition 15, they are self-contained, strongly depleting and justificationpreserving. They are, however, generally not minimal, even amongst strongly depleting and self-contained modules.

\section{Overview}

We now provide a high-level overview of our approach to module extraction, which is based on a novel reduction to a reasoning problem in datalog. Our approach builds on recent techniques that exploit datalog engines for ontology reasoning (Kontchakov, Lutz, Toman, Wolter, \& Zakharyaschev, 2011; Stefanoni, Motik, \& Horrocks, 2013; Zhou, Nenov, Cuenca Grau, \& Horrocks, 2014; Zhou, Cuenca Grau, Nenov, Kaminski, \& Horrocks, 2015). The connection between module extraction and datalog was first observed in (Suntisrivaraporn, 2008), where it was shown that $\perp$-module extraction for the lightweight $\mathrm{DL} \mathcal{E L}^{+}$can be reduced to propositional datalog reasoning.

Our approach takes this connection much farther by providing a unified framework that supports module extraction for arbitrary ontologies consisting of first-order rules, as well as for a wide range of inseparability relations. Modules obtained using our approach can be tailored to the requirements of the application at hand. In addition to being significantly smaller in practice, our modules preserve the features of syntactic locality modules: they are widely applicable, they can be efficiently computed in practice, and they satisfy a wide range of additional properties.

In what follows, we fix w.l.o.g. an arbitrary ontology $\mathcal{O}$ and a signature $\Sigma \subseteq \operatorname{Sig}(\mathcal{O})$. Unless otherwise stated, our definitions and theorems are parameterised by such $\mathcal{O}$ and $\Sigma$. As stated in Section 2, we assume that rules in $\mathcal{O}$ do not share variables. 
Our overall strategy to extract a module $\mathcal{M}$ of $\mathcal{O}$ can be roughly summarised by the following steps: ${ }^{1}$

1. Choose a substitution $\theta$ mapping all existentially quantified variables in $\mathcal{O}$ to fresh Skolem constants, and obtain a datalog program $\mathcal{P}$ from $\mathcal{O}$ by

(a) Skolemising all rules in $\mathcal{O}$ using $\theta$ to obtain function-free rules $\varphi \rightarrow \psi$, which may contain both $\wedge$ and $\vee$ in the head; and

(b) replacing each of the resulting rules $\varphi \rightarrow \psi$ with the set $\{\varphi \rightarrow \gamma \mid \gamma$ an atom in $\psi\}$ of datalog rules; in this way, disjunctions in the head of rules are turned into conjunctions and split into different datalog rules.

Clearly, such a program $\mathcal{P}$ logically entails $\mathcal{O}$ and thus preserves all its consequences.

2. Choose a $\Sigma$-dataset $\mathcal{D}_{0}$ of "initial facts" and compute the materialisation of $\mathcal{P} \cup \mathcal{D}_{0}$.

3. Choose a set $\mathcal{D}_{r}$ of "relevant facts" in the materialisation (possibly containing symbols outside $\Sigma$ ), and compute the supporting rules $\mathcal{P}^{\prime}$ in $\mathcal{P}$ for each such fact.

4. Output the subset $\mathcal{M} \subseteq \mathcal{O}$ of all rules in $\mathcal{O}$ that correspond to some rule in $\mathcal{P}^{\prime}$.

The subset $\mathcal{M}$ described above is fully determined by the substitution $\theta$ and the datasets $\mathcal{D}_{0}$ and $\mathcal{D}_{r}$. The main intuition behind our module extraction approach is that we can pick $\theta, \mathcal{D}_{0}$ and $\mathcal{D}_{r}$ (and hence also $\mathcal{M}$ ) such that each proof $\rho$ of a $\Sigma$-consequence $\varphi$ of $\mathcal{O}$ to be preserved under the inseparability relation of interest can be embedded into a collection of proofs in $\mathcal{P} \cup \mathcal{D}_{0}$ of a relevant fact from $\mathcal{D}_{r}$. In this way, we can ensure that $\mathcal{M}$ contains all the necessary rules to entail $\varphi$.

Example 18. To illustrate how our strategy might work in practice, consider our running example ontology $\mathcal{O}^{e x}$ from Figure 3 and signature $\Sigma=\{B, C, D, H\}$.

Assume that our goal is to compute a module $\mathcal{M}$ that is $\Sigma$-implication inseparable from $\mathcal{O}^{e x}$. Recall from Example 6 that the sentence $\varphi=D(x) \rightarrow H(x)$ is the only non-trivial $\Sigma$-implication entailed by $\mathcal{O}^{e x}$, and therefore the only requirement for $\mathcal{M}$ is that $\mathcal{M} \models \varphi$. Furthermore, note that proving $\mathcal{O}^{e x} \models \varphi$ amounts to proving $\mathcal{O}^{e x} \cup\{D(a)\} \models H(a)$ with $a$ some fresh constant (cf. Proposition 1 in Section 2.1).

Figure 4(a) depicts a hyperresolution proof $\rho$ showing how $H(a)$ can be derived from $D(a)$ and the set of clauses corresponding to $r_{5}-r_{8}$, where rule $r_{6}$ is transformed into the clause $r_{6}^{\kappa}=F(x) \rightarrow S\left(x, f_{y_{2}}^{r_{6}}(x)\right)$. It follows that $\mathcal{M}=\left\{r_{5}-r_{8}\right\}$ is $\Sigma$-implication inseparable from $\mathcal{O}^{e x}$ since it covers the support of $\rho$. Moreover, $\mathcal{M}$ is minimal since $H(a)$ cannot be derived from any subset of $\left\{r_{5}-r_{8}\right\}$.

In our approach, we take $\mathcal{D}_{0}$ and $\mathcal{D}_{r}$ to contain, respectively, the initial fact $D(a)$ and the fact $H(a)$ to be proved. We also make $\theta$ map variables $y_{1}$ and $y_{2}$ to fresh constants $c_{y_{1}}$ and $c_{y_{2}}$, respectively. The resulting datalog program $\mathcal{P}$ is shown in Figure 5.

Figure 4(b) depicts proofs $\rho^{\prime}$ and $\rho^{\prime \prime}$ of $H(a)$ in $\mathcal{P} \cup\{D(a)\}$. The support of proof $\rho^{\prime \prime}$ in the datalog program consists of rules $r_{5}^{\prime \prime}$ and $r_{8}$, which stem from rules $r_{5}$ and $r_{8}$ in $\mathcal{O}^{e x}$; we

1. For simplicity, in this section we overlook certain technical details such as the presence of constants in $\mathcal{O}$. These will be thoroughly addressed later on. 


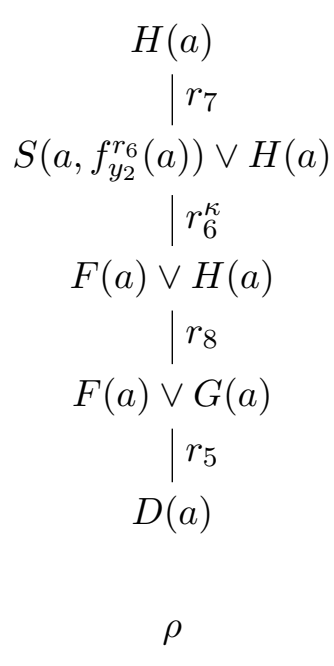

(a)

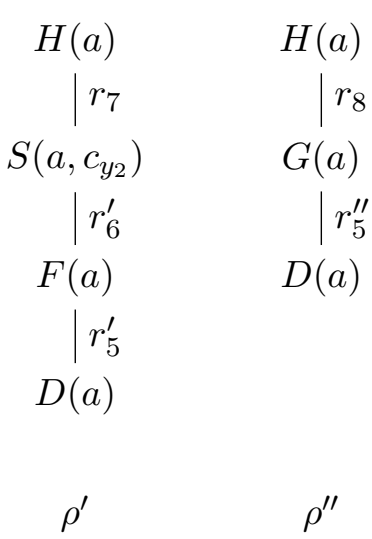

(b)

Figure 4: Proofs of $H(a)$ from $D(a)$ in (a) $\mathcal{O}^{e x}$ and (b) the corresponding datalog program

$$
\begin{aligned}
& r_{1}^{\prime}: A(x) \rightarrow R\left(x, c_{y_{1}}\right) \quad r_{1}^{\prime \prime}: \quad A(x) \rightarrow B\left(c_{y_{1}}\right) \\
& r_{2}: A(x) \rightarrow R(x, o) \\
& r_{3}: \quad B(x) \wedge C(x) \rightarrow D(x) \\
& r_{4}: \quad R(x, y) \wedge C(y) \rightarrow E(x) \\
& r_{5}^{\prime}: \quad D(x) \rightarrow F(x) \quad r_{5}^{\prime \prime}: \quad D(x) \rightarrow G(x) \\
& r_{6}^{\prime}: \quad F(x) \rightarrow S\left(x, c_{y_{2}}\right) \\
& r_{7}: \quad S(x, y) \rightarrow H(x) \\
& r_{8}: \quad G(x) \rightarrow H(x)
\end{aligned}
$$

Figure 5: Datalog program obtained from $\mathcal{O}^{e x}$ using $\theta=\left\{y_{1} \mapsto c_{y_{1}}, y_{2} \mapsto c_{y_{2}}\right\}$

can see, however, that $\left\{r_{5}, r_{8}\right\} \subsetneq \mathcal{M}$ and hence $\mathcal{M}$ does not entail $\varphi$. The same situation arises if we were to consider $\rho^{\prime}$ only, in which case we would recover only rules $r_{5}-r_{7}$. This is because the datalog program is a strengthening of $\mathcal{O}^{e x}$ and one particular proof in the datalog program may not translate back into a proof over the original ontology. Indeed, in order to compute $\mathcal{M}$, we need to consider the supports of both $\rho^{\prime}$ and $\rho^{\prime \prime}$, in which case we would successfully recover $\mathcal{M}$.

In this example, our approach would allow us to compute a minimal module. This is, however, not the case in general: since $\mathcal{P}$ is a strengthening of the given ontology there may be proofs in $\mathcal{P} \cup \mathcal{D}_{0}$ of facts in $\mathcal{D}_{r}$ that do not correspond to proofs of any $\Sigma$-consequence of the ontology, which may then lead to the inclusion of unnecessary rules in the module.

In the following sections we describe our approach formally. 
- In Section 5, we define the general notion of a module setting, which captures the degrees of freedom of our framework and uniquely specifies the datalog program $\mathcal{P}$ and module $\mathcal{M}$ corresponding to specific choices of $\theta, \mathcal{D}_{0}$, and $\mathcal{D}_{r}$. Furthermore, we establish the key correspondence between proofs over the original ontology $\mathcal{O}$ and sets of proofs over $\mathcal{P} \cup \mathcal{D}_{0}$, which we exploit in many of our subsequent technical results.

- In Section 6, we describe concrete module settings for each of the inseparability relations introduced in Section 3.1, namely implication (Section 6.1), fact (Section 6.2), query (Section 6.3), and model inseparability (Section 6.4) where we also show that locality $\perp$-modules can be precisely captured by an instantiation of our framework.

- In Section 7, we consider variants of the inseparability relations in Section 3.1 studied in the literature, and describe specific module settings for them. These results show that our framework can be easily adapted to capture new inseparability relations and hence illustrate the generality and versatility of our approach.

- In Section 8, we show that our modules are consistent with the intuition that stronger inseparability relations should lead to larger modules. For this, we introduce a notion of homomorphism between module settings, which will allow us to establish containment relations between the modules specified in Sections 6 and 7 .

- In Section 9, we study the additional properties of our modules. We show that they are depleting and justification-preserving for all the inseparability relations in previous sections. Our modules, however, may not be strongly depleting or self-contained; although this may be beneficial, as it allows us to extract smaller modules, these properties are still important for ontology reuse scenarios. Hence, we propose a technique that ensures that extracted modules are also strongly depleting and self-contained.

- In Section 10, we briefly discuss the complexity of module extraction within our framework and show tractability for DL-based ontology languages.

- Finally, in Section 11, we discuss the optimality of the module settings introduced in Sections 6 and 7. In particular, although our modules are not minimal in general, we aim at determining whether the modules obtained from the settings in Sections 6 and 7 are the smallest possible within our framework.

\section{The Notion of a Module Setting}

In this section we present our framework for module extraction. The key notion is that of a module setting, which captures in a declarative way the main elements of our approach discussed in Section 4.

Definition 19. A module setting for $\mathcal{O}$ and $\Sigma$ is a tuple $\chi=\left\langle\theta, \mathcal{D}_{0}, \mathcal{D}_{r}\right\rangle$ with

- $\theta$ a substitution mapping each constant and existentially quantified variable in $\mathcal{O}$ to a (possibly fresh) constant;

- $\mathcal{D}_{0}$ a dataset mentioning only predicates from $\Sigma$; and 
- $\mathcal{D}_{r}$ a dataset mentioning only predicates from $\operatorname{Sig}(\mathcal{O}) \cup\{\perp\}$.

For each rule $r=\varphi(\mathbf{x}) \rightarrow \exists \mathbf{y} \psi(\mathbf{x}, \mathbf{y})$ in $\mathcal{O}$, let

$$
\Xi^{\chi}(r)=\{(\varphi \rightarrow \gamma) \theta \mid \gamma \text { an atom in } \psi\}
$$

The program $\mathcal{P}^{\chi}$ of $\chi$ is defined as

$$
\mathcal{P}^{\chi}=\bigcup_{r \in \mathcal{O}} \Xi^{\chi}(r)
$$

and the support of $\chi$ is the set

$$
\operatorname{supp}(\chi)=\left\{r \mid r \in \operatorname{supp}(\rho) \text { with } \rho \text { a proof in } \mathcal{P}^{\chi} \cup \mathcal{D}_{0} \text { of a fact from } \mathcal{D}_{r}\right\} .
$$

Finally, if $\mathcal{F}=\left\{r \in \mathcal{O} \mid \operatorname{supp}(\chi) \cap \Xi^{\chi}(r) \neq \emptyset\right\}$, the module $\mathcal{M}^{\chi}$ of $\chi$ is defined as the following subset of $\mathcal{O}$ :

$$
\mathcal{M}^{\chi}=\mathcal{F} \cup \mathcal{F}^{\perp} \cup \mathcal{F}^{\top} \cup \mathcal{F}^{\approx}
$$

The mapping $\theta$ and the datasets $\mathcal{D}_{0}$ and $\mathcal{D}_{r}$ constitute the degrees of freedom of our framework, and Definition 19 ensures that specific choices of these parameters of the module setting $\chi$ fully determine the module $\mathcal{M}^{\chi}$.

The datalog program $\mathcal{P} \chi$ is obtained by applying $\theta$ to each rule $r$ in $\mathcal{O}$ while at the same time splitting the head atoms of $r$ into different rules. The application of $\theta$ turns existentially quantified variables into (possibly fresh) constants and hence transforms $\mathcal{O}$ into a set of rules where all variables are universally quantified; additionally, $\theta$ maps the constants occurring in $\mathcal{O}$ to (possibly different) constants. Since $\theta$ is not required to be injective, it is possible for $\theta$ to map an existentially quantified variable and a constant from $\mathcal{O}$ to the same constant. As we will see next, $\mathcal{P}^{\chi}$ is a strengthening of $\mathcal{O}$ in the sense that it preserves all consequences of $\mathcal{O}$ when coupled with an arbitrary dataset. Analogous datalog strengthenings have been exploited to overestimate reasoning outcomes in description logic ontologies (Krötzsch, Rudolph, \& Hitzler, 2008b; Stefanoni et al., 2013; Krötzsch, Rudolph, \& Hitzler, 2008a; Zhou et al., 2014; Zhou, Nenov, Cuenca Grau, \& Horrocks, 2013; Zhou, Cuenca Grau, Horrocks, Wu, \& Banerjee, 2013).

The support $\operatorname{supp}(\chi)$ collects all datalog rules participating in any proof in $\mathcal{P}^{\chi} \cup \mathcal{D}_{0}$ of any relevant fact from $\mathcal{D}_{r}$. Intuitively, this support captures the "image" of the module in $\mathcal{P}^{\chi}$. Finally, the module $\mathcal{M}^{\chi}$ consists of all the rules in $\mathcal{O}$ that have a corresponding datalog rule in the support $\operatorname{supp}(\chi)$.

Example 20. Let us reconsider Example 18 in Section 4, where we chose $\theta$ to map variables $y_{1}$ and $y_{2}$ to fresh constants $c_{y_{1}}$ and $c_{y_{2}}$, whereas $\mathcal{D}_{0}$ and $\mathcal{D}_{r}$ contain, respectively, the initial fact $D(a)$ and the fact $H(a)$ to be proved. Definition 19 ensures that $\mathcal{P}^{\chi}$ consists precisely of the datalog rules in Figure 5. The support $\operatorname{supp}(\chi)$ consists of all rules in the support of $\rho^{\prime}$ and $\rho^{\prime \prime}$ shown in Figure 4. Finally, $\mathcal{M}^{\chi}$ consists of rules $r_{5}-r_{8}$, as required.

The following lemma establishes a key correspondence between hyperresolution proofs in (the clausification of) $\mathcal{O}$ and sets of proofs in the datalog program $\mathcal{P}^{\chi}$. Such a correspondence is already manifest in Figure 4 for our running example. Given an arbitrary dataset 
$\mathcal{D}$ and a substitution $\tau$ from constants to constants that is compatible with $\theta$ in $\chi$ (i.e., $\tau$ and $\theta$ coincide over the intersection of their domains), the lemma shows that each proof $\rho$ of a disjunction of facts $\varphi=\bigvee_{i=1}^{n} \gamma_{i}$ in $\kappa(\mathcal{O}) \cup \mathcal{D}$ has a corresponding set of proofs $\mathcal{T}$ of each disjunct $\gamma_{i} \tau$ in $\mathcal{P}^{\chi} \cup \mathcal{D} \tau$. This implies, in particular, that $\mathcal{P}^{\chi}$ is indeed a strengthening of $\mathcal{O}$. Furthermore, the set $\mathcal{T}$ is both support and structure preserving: for every clause from $\kappa(r)$ participating in $\rho$, there is a proof in $\mathcal{T}$ with a datalog rule from $\Xi^{\chi}(r)$ in its support; finally, each proof in $\mathcal{T}$ is embeddable into $\rho$ and hence its structure is compatible with that of $\rho$ (cf. Section 2.3). ${ }^{2}$

Lemma 21. Let $\chi$ be a module setting for $\mathcal{O}$ and $\Sigma$ and let its corresponding substitution be $\theta$. Let $\mathcal{D}$ be a dataset and $\tau$ an arbitrary substitution from constants in $\mathcal{D}$ into constants that is compatible with $\theta$. Finally, let $\varphi$ be a (possibly empty) disjunction of facts and $\rho=(T, \lambda)$ a proof of $\varphi$ in $\kappa(\mathcal{O}) \cup \mathcal{D}$. Then there exists a non-empty set $\mathcal{T}$ of proofs in $\mathcal{P} \chi \cup \mathcal{D} \tau$ satisfying the following properties:

1. Each $\rho^{\prime} \in \mathcal{T}$ is a proof of $\gamma \tau$ for some $\gamma \in \varphi \cup\{\perp\}$. Furthermore, for each $\gamma \in \varphi$ there is some proof of $\gamma \tau$ in $\mathcal{T}$.

2. For each $r \in \mathcal{O}$ with $\kappa(r) \cap \operatorname{supp}(\rho) \neq \emptyset$, either $r=\perp \rightarrow \square$ or there exists $\rho^{\prime} \in \mathcal{T}$ with $\Xi^{\chi}(r) \cap \operatorname{supp}\left(\rho^{\prime}\right) \neq \emptyset$.

3. Each $\rho^{\prime} \in \mathcal{T}$ is embeddable into $\rho$ modulo $\tau \cup \theta$.

Proof. To prove these results it suffices to show that

(a) for each $\gamma \in \varphi$ there exists a proof $\rho^{\prime}$ in $\mathcal{P}^{\chi} \cup \mathcal{D} \tau$ of $\gamma \tau$ that is embeddable into $\rho$ modulo $\tau \cup \theta$, and

(b) for each $r \in \mathcal{O}$ s.t. $\kappa(r) \cap \operatorname{supp}(\rho) \neq \emptyset$, either $r=\perp \rightarrow \square$ or there exists $\gamma \in \varphi \cup\{\perp\}$ and a proof $\rho^{\prime}$ of $\gamma \tau$ in $\mathcal{P}^{\chi} \cup \mathcal{D} \tau$ that is embeddable into $\rho$ modulo $\tau \cup \theta$, and such that $\Xi^{\chi}(r) \cap \operatorname{supp}\left(\rho^{\prime}\right) \neq \emptyset$.

In order to be able to reason by induction on the depth $d$ of $\rho$, we prove that the above properties hold even if $\varphi$ is a disjunction of (not necessarily function-free) ground atoms.

$d=0$

If $\operatorname{supp}(\rho)=\emptyset$ then $\varphi$ is a fact in $\mathcal{D}$ and $\varphi(\tau \cup \theta)=\varphi \tau \in \mathcal{D} \tau$ so there exists a trivial proof $\rho^{\prime}$ in $\mathcal{P}^{\chi} \cup \mathcal{D} \tau$ of $\varphi(\tau \cup \theta)$, which is clearly embeddable into $\rho$ via $\tau \cup \theta$.

If $\operatorname{supp}(\rho) \neq \emptyset$ then $\kappa(\mathcal{O})$ must contain a clause of the form $(\rightarrow \varphi)$. Since, by assumption, the only rule in $\mathcal{O}$ with an empty head is $\perp \rightarrow \square$, it must be the case that $\varphi \neq \square$ and for each $\gamma \in \varphi$ there exists a proof $\rho^{\prime}$ in $\mathcal{P}^{\chi} \cup \mathcal{D} \tau$ of $\gamma \theta=\gamma(\tau \cup \theta)$ of depth 0 that is supported by $(\rightarrow \gamma \theta) \in \Xi^{\chi}(r)$ and embeddable into $\rho$ modulo $\tau \cup \theta$.

In either case, both properties are satisfied.

2. In Lemma 21, as well as in some of our subsequent technical results, we prove statements about $\kappa(\mathcal{O})$ rather than about $\mathcal{O}$. In such cases, we consider an extension of $\theta$ in $\chi$ where functional terms $f_{y}^{r}(\mathbf{t})$ occurring in $\kappa(\mathcal{O})$ are mapped to $y \theta$ whenever $y$ is in the domain of $\theta$; by slight abuse of notation and for the sake of simplicity, we also refer to such an extended substitution as $\theta$. 
$d>0$

Let $\rho=(T, \lambda)$ with $v$ the root of $T$ and $w_{1}, \ldots, w_{n}$ the children of $v$. Consider a clause $s \in \kappa(\mathcal{O})$ such that $\varphi$ is a hyperresolvent of $s$ and $\lambda\left(w_{1}\right), \ldots, \lambda\left(w_{n}\right)$. Then, $s$ must be of the form $\bigwedge_{i=1}^{n} \delta_{i}^{\prime} \rightarrow \varphi^{\prime}$ where

$-\lambda\left(w_{i}\right)=\delta_{i} \vee \psi_{i}$ for each $1 \leq i \leq n$, and

$-\varphi=\bigvee_{i=1}^{n} \psi_{i} \vee \varphi^{\prime} \sigma$ with $\sigma$ a MGU of $\delta_{i}, \delta_{i}^{\prime}$ for each $1 \leq i \leq n$.

(a) Let $\gamma \in \varphi$. We need to find a proof $\rho^{\prime}=\left(T^{\prime}, \lambda^{\prime}\right)$ of $\gamma(\tau \cup \theta)$ in $\mathcal{P}^{\chi} \cup \mathcal{D} \tau$ that is embeddable into $\rho$ modulo $\tau \cup \theta$. If $\gamma \in \psi_{i}$ then by induction hypothesis we can find such a proof. If $\gamma \in \varphi^{\prime} \sigma$ then it holds that $\gamma=\gamma^{\prime} \sigma$ for some $\gamma^{\prime} \in \varphi^{\prime}$. By induction hypothesis, we have a proof $\rho_{i}=\left(T_{i}, \lambda_{i}\right)$ in $\mathcal{P}^{\chi} \cup \mathcal{D} \tau$ of each $\delta_{i}(\tau \cup \theta)$ that is embeddable modulo $\tau \cup \theta$ into a proper subproof of $\rho$. Because $\sigma$ is a MGU of $\delta_{i}$ and $\delta_{i}^{\prime}$, with $\delta_{i}=\delta_{i}^{\prime} \sigma$, we have that $\sigma(\tau \cup \theta)$ is a MGU of of $\delta_{i}(\tau \cup \theta)$ and $\delta_{i}^{\prime} \theta$. Indeed, $\delta_{i}=\delta_{i}^{\prime} \sigma$ implies $\delta_{i}(\tau \cup \theta)=\left(\delta_{i}^{\prime} \sigma\right)(\tau \cup \theta)$; on the other hand, since the effect of $\theta$ on each functional term $f(\mathbf{t})$ does not depend of $\mathbf{t}$, we have $\left(\delta_{i}^{\prime} \sigma\right)(\tau \cup \theta)=\left(\delta_{i}^{\prime}(\tau \cup \theta)\right)(\sigma(\tau \cup \theta))$; moreover, because $\tau \cup \theta$ only extends the domain of $\theta$ to constants in $\mathcal{D}$ but not in $\mathcal{O}$, we have $\left(\delta_{i}^{\prime}(\tau \cup \theta)\right)(\sigma(\tau \cup \theta))=\left(\delta_{i}^{\prime} \theta\right)(\sigma(\tau \cup \theta))$, and thus $\delta_{i}(\tau \cup \theta)=\left(\delta_{i}^{\prime} \theta\right)(\sigma(\tau \cup \theta))$. We can hence combine all the $\rho_{i}$ with $\left(\bigwedge_{i=1}^{n} \delta_{i}^{\prime} \rightarrow \gamma^{\prime}\right) \theta \in \mathcal{P} \chi$, to obtain a proof of $\left(\gamma^{\prime} \theta\right)(\sigma(\tau \cup \theta))=\left(\gamma^{\prime} \sigma\right)(\tau \cup \theta)=\gamma(\tau \cup \theta)$ in $\mathcal{P}^{\chi} \cup \mathcal{D} \tau$ that is clearly also embeddable into $\rho$ modulo $\tau \cup \theta$.

(b) Consider $r \in \mathcal{O}$ such that there exists $r^{\prime} \in \kappa(r) \cap \operatorname{supp}(\rho)$. We need to find $\gamma \in \varphi \cup\{\perp\}$ and a proof $\rho^{\prime}=\left(T^{\prime}, \lambda^{\prime}\right)$ of $\gamma(\tau \cup \theta)$ in $\mathcal{P}^{\chi} \cup \mathcal{D} \tau$ that is embeddable into $\rho$ modulo $\tau \cup \theta$ and has some rule from $\Xi^{\chi}(r)$ in its support.

Assume first that $r^{\prime}=s$ and $r \neq \perp \rightarrow \square$. Then it must be $\varphi^{\prime} \neq \square$ since, by assumption, $\perp \rightarrow \square$ is the only rule in $\mathcal{O}$ with an empty head. We can then pick any $\gamma^{\prime} \in \varphi^{\prime}$ and have $\rho^{\prime}$ be a proof of $\left(\gamma^{\prime} \sigma\right)(\tau \cup \theta)$ in $\mathcal{P}^{\chi} \cup \mathcal{D} \tau$ that is supported by $\left(\bigwedge_{i=1}^{n} \delta_{i}^{\prime} \rightarrow \gamma^{\prime}\right) \theta \in \Xi^{\chi}(r)$, as we saw when considering property (a).

Assume now that $r^{\prime} \neq s$ and $r \neq \perp \rightarrow \square$. Then there must be some $i$ such that $r^{\prime}$ supports a proof $\tilde{\rho}_{i}$ of $\delta_{i} \vee \psi_{i}$ that is a subproof of $\rho$. Since $\tilde{\rho}_{i}$ is of depth $<d$, by i.h. there must be some $\delta_{i}^{\prime \prime} \in \delta_{i} \vee \psi_{i} \cup\{\perp\}$ and a proof $\rho_{i}^{\prime \prime}=\left(T^{\prime \prime}, \lambda^{\prime \prime}\right)$ of $\delta_{i}^{\prime \prime}(\tau \cup \theta)$ in $\mathcal{P} \chi \cup \mathcal{D} \tau$ that is supported by some rule in $\Xi^{\chi}(r)$ and is embeddable into $\tilde{\rho}_{i}$. If $\delta_{i}^{\prime \prime} \in \psi_{i} \cup\{\perp\} \subseteq \varphi \cup\{\perp\}$ then $\rho^{\prime}=\rho^{\prime \prime}$ is the proof we are looking for. If $\delta_{i}^{\prime \prime}=\delta_{i}$ (and $\delta_{i} \neq \perp$ ) we can combine $\rho^{\prime \prime}$ with suitable proofs of each $\delta_{j}(\tau \cup \theta)$ for the remaining $j$ (which we know exist by i.h.), as before, to construct a proof $\rho^{\prime}$ in $\mathcal{P} \chi \cup \mathcal{D} \tau$ of $\gamma(\tau \cup \theta)$ for some $\gamma \in \varphi$, that is embeddable into $\rho$ modulo $\tau \cup \theta$.

Lemma 21 and Proposition 1 establish how the datasets $\mathcal{D}_{0}$ and $\mathcal{D}_{r}$ in $\chi$ can be chosen so as to ensure that $\mathcal{M}^{\chi}$ preserves the required $\Sigma$-consequences.

Suppose that $\mathcal{M}^{\chi}$ is required to preserve some $\Sigma$-consequence $r=\varphi(\mathbf{x}) \rightarrow \exists \mathbf{y} \psi(\mathbf{x}, \mathbf{y})$ of $\mathcal{O}$. By Proposition 1, given any substitution mapping variables in $\mathbf{x}$ to distinct constants $\mathbf{c}$, it must be the case that $\mathcal{O} \cup \varphi(\mathbf{c}) \models \exists \mathbf{y} \psi(\mathbf{c}, \mathbf{y})$. Since $\mathcal{P} \chi$ is a strengthening of $\mathcal{O}$ we also have $\mathcal{P}^{\chi} \cup \varphi(\mathbf{c}) \models \exists \mathbf{y} \psi(\mathbf{c}, \mathbf{y})$. Assume that we now choose $\mathcal{D}_{0}$ and $\mathcal{D}_{r}$ so as to satisfy the following requirements: 
1. the instantiation $\varphi(\mathbf{c})$ of the body of $r$ must be embeddable in $\mathcal{D}_{0}$; and

2. the set of all facts $\gamma$ in the materialisation of $\mathcal{P}^{\chi} \cup \varphi(\mathbf{c})$ satisfying the instantiation $\exists \mathbf{y} \psi(\mathbf{c}, \mathbf{y})$ of the head of $r$ must be embeddable into $\mathcal{D}_{r}$.

By completeness of hyperresolution, and given that $\mathcal{P}^{\chi}$ is a datalog program, there must exist a fact $\gamma$ s.t. $\gamma \models \exists \mathbf{y} \psi(\mathbf{c}, \mathbf{y})$ and $\mathcal{P}^{\chi} \cup \varphi(\mathbf{c}) \vdash \gamma$. By Lemma 21 and Definition 19, the aforementioned requirements on $\mathcal{D}_{0}$ and $\mathcal{D}_{r}$ suffice to guarantee that $\mathcal{M}^{\chi}$ will preserve the consequence $r$.

Example 22. Consider again our running example in Section 4 and the associated module setting $\chi=\left\langle\theta, \mathcal{D}_{0}, \mathcal{D}_{r}\right\rangle$ given in Example 20. We can see that our choices of $\mathcal{D}_{0}=\{D(a)\}$ and $\mathcal{D}_{r}=\{H(a)\}$ satisfy our sufficient requirements for $\mathcal{M}^{\chi}$ to entail $\varphi=D(x) \rightarrow H(x)$. First, any instantiation of the body $D(x)$ of $\varphi$ is isomorphic to (and hence embeddable into) $\mathcal{D}_{0}$. Second, the materialisation of $\mathcal{P} \chi \cup\{D(a)\}$ consists of facts $F(a), S\left(a, c_{y_{2}}\right)$ and $H(a)$, where the latter is isomorphic to the instantiation of the head of $\varphi$; since we chose $\mathcal{D}_{r}$ to consist precisely of $H(a)$, the second requirement is also satisfied.

The following theorem makes precise the aforementioned sufficient requirements for $\mathcal{M}^{\chi}$ to preserve the entailment of a $\Sigma$-rule $r$. Furthermore, it shows that whenever $\mathcal{M}^{\chi}$ entails $r$, it also contains all the justifications for $r$ in the original ontology $\mathcal{O}$.

Theorem 23. Let $r=\varphi(\mathbf{x}) \rightarrow \exists \mathbf{y} \psi(\mathbf{x}, \mathbf{y})$ be a rule and $\chi=\left\langle\theta, \mathcal{D}_{0}, \mathcal{D}_{r}\right\rangle$ a module setting for $\mathcal{O}$ and $\Sigma$ such that for each substitution $\sigma$ mapping all variables in $\mathbf{x}$ to pairwise distinct constants, there exists another substitution $\tau_{\sigma}$ that is compatible with $\theta$ and such that

- $(\varphi \sigma) \tau_{\sigma} \subseteq \mathcal{D}_{0}$, and

- $\left((\psi \sigma) \tau_{\sigma}\right) \sigma^{\prime} \cup\{\perp\} \subseteq \mathcal{D}_{r}$ for each substitution $\sigma^{\prime}$ mapping variables in $\mathbf{y}$ to constants and such that $\mathcal{P} \chi \cup(\varphi \sigma) \tau_{\sigma}=\left((\psi \sigma) \tau_{\sigma}\right) \sigma^{\prime}$.

Then $\mathcal{O}=r$ iff $\mathcal{M}^{\chi} \models r$. Furthermore, if $\mathcal{O}^{\prime}$ is a justification for $r$ in $\mathcal{O}$, then $\mathcal{O}^{\prime} \subseteq \mathcal{M}^{\chi}$.

Proof. Since $\mathcal{M}^{\chi} \subseteq \mathcal{O}$, it follows from monotonicity of first-order logic that $\mathcal{O} \models r$ whenever $\mathcal{M}^{\chi}=r$. To prove the opposite direction of the implication, it suffices to show that if $\mathcal{O}^{\prime} \subseteq \mathcal{O}$ is a justification for $r$ in $\mathcal{O}$, then $\mathcal{O}^{\prime} \subseteq \mathcal{M}^{\chi}$.

If $\psi=\square$ then, by minimality of $\mathcal{O}^{\prime}$, given a substitution $\sigma$ mapping variables in $\mathbf{x}$ to fresh distinct constants, there must be a proof $\rho$ of $\square$ in $\kappa(\mathcal{O}) \cup \varphi \sigma$ such that $\operatorname{supp}(\rho) \cap \kappa(r) \neq \emptyset$ for each $r \in \mathcal{O}^{\prime}$. By assumption, there exists a substitution $\tau_{\sigma}$ that is compatible with $\theta$ and such that $(\varphi \sigma) \tau_{\sigma} \subseteq \mathcal{D}_{0}$. By Lemma 21, for each $r \in \mathcal{O}^{\prime}$ either $r=\perp \rightarrow \square$ or there exists a proof $\rho_{\perp}$ in $\mathcal{P} \chi \cup(\varphi \sigma) \tau_{\sigma}$ of $\perp$ such that $\operatorname{supp}\left(\rho_{\perp}\right) \cap \Xi^{\chi}(r) \neq \emptyset$. If $r=\perp \rightarrow \square$ then, since by assumption the only rule in $\mathcal{O}$ with an empty head is $\perp \rightarrow \square$, in particular it must also be the case that $\mathcal{O}=\varphi(\mathbf{x}) \rightarrow \perp$. It suffices to show that in this case also $\mathcal{M}^{\chi} \models \varphi(\mathbf{x}) \rightarrow \perp$ (as we do next when considering $\psi \neq \square$ ): then, it follows that $\perp \in \operatorname{Sig}\left(\mathcal{M}^{\chi}\right)$ and therefore $r=\perp \rightarrow \square \in \mathcal{M}^{\chi}$ because $\mathcal{M}^{\chi}$ is an ontology. If $r \neq \perp \rightarrow \square$ then, since $(\varphi \sigma) \tau_{\sigma} \subseteq \mathcal{D}_{0}$ and $\perp \in \mathcal{D}_{r}$, it follows that $r \in \mathcal{M}^{\chi}$.

If $\psi \neq \square$ we can assume w.l.o.g. that $\psi=\bigvee_{i=1}^{n} \psi_{i}$ with $m>0$ and each $\psi_{i}$ a conjunction of atoms. For a fresh predicate $Q$, consider the ontology

$$
\mathcal{O}_{Q}=\left\{\psi_{i}(\mathbf{x}, \mathbf{y}) \rightarrow Q(\mathbf{x}) \mid 1 \leq i \leq n\right\}
$$


It is immediate that, for each subset $\mathcal{O}^{\prime \prime} \subseteq \mathcal{O}$, it is $\mathcal{O}^{\prime \prime} \models r$ iff $\mathcal{O}^{\prime \prime} \cup \mathcal{O}_{Q} \models \varphi(\mathbf{x}) \rightarrow Q(\mathbf{x})$. Therefore, by minimality of $\mathcal{O}^{\prime}$, there must be some $\mathcal{O}_{Q}^{\prime} \subseteq \mathcal{O}_{Q}$ such that $\mathcal{O}^{\prime} \cup \mathcal{O}_{Q}^{\prime}$ is a justification of $\varphi(\mathbf{x}) \rightarrow Q(\mathbf{x})$ in $\mathcal{O} \cup \mathcal{O}_{Q}$. By minimality of $\mathcal{O}^{\prime} \cup \mathcal{O}_{Q}^{\prime}$, given a substitution $\sigma$ mapping variables in $\mathbf{x}$ to fresh distinct constants, there must be a proof $\rho$ of $Q(\mathbf{x}) \sigma$ in $\kappa\left(\mathcal{O} \cup \mathcal{O}_{Q}\right) \cup \varphi \sigma$ such that $\kappa(r) \cap \operatorname{supp}(\rho) \neq \emptyset$ for each $r \in \mathcal{O}^{\prime} \cup \mathcal{O}_{Q}^{\prime}$.

By assumption, there is a substitution $\tau_{\sigma}$ that is compatible with $\theta$ and such that $(\varphi \sigma) \tau_{\sigma} \subseteq \mathcal{D}_{0}$. Since $\mathcal{O}_{Q}$ does not contain any existentially quantified variables, or any constants that do not occur already in $\mathcal{O}$, there exists a module setting $\chi_{Q}=\left\langle\theta^{Q}, \mathcal{D}_{0}^{Q}, \mathcal{D}_{r}^{Q}\right\rangle$ for $\mathcal{O} \cup \mathcal{O}_{Q}$ and $\Sigma$ such that $\theta^{Q}=\theta$, and therefore $\mathcal{P}^{\chi_{Q}}=\mathcal{P}^{\chi} \cup \mathcal{O}_{Q}$. By Lemma 21, for each $s \in \mathcal{O}^{\prime} \subseteq \mathcal{O}^{\prime} \cup \mathcal{O}_{Q}^{\prime}$ either it is $s=\perp \rightarrow \square$ or there exists a proof $\rho^{\prime}$ in $\mathcal{P}^{\chi} Q \cup(\varphi \sigma) \tau_{\sigma}$ of either $(Q(\mathbf{x}) \sigma) \tau_{\sigma}$ or $\perp$ such that $s^{\prime} \in \Xi^{\chi Q}(s) \cap \operatorname{supp}\left(\rho^{\prime}\right) \neq \emptyset$.

If $s=\perp \rightarrow \square$ then there must be some proof in $\kappa\left(\mathcal{O} \cup \mathcal{O}_{Q}\right) \cup \varphi \sigma$ of a disjunction of the form $\perp \vee \phi$ (with $\phi$ possibly empty). By Lemma 21 there exists a proof $\rho_{\perp}$ in $\mathcal{P} \chi_{Q} \cup(\varphi \sigma) \tau_{\sigma}$ of $\perp$. Furthermore, since $\perp$ does not mention $Q$, and neither does the body of any rule in $\mathcal{P} \chi_{Q}=\mathcal{P} \chi \cup \mathcal{O}_{Q}$, the proof $\rho_{\perp}$ must actually be a proof in $\mathcal{P} \chi \cup(\varphi \sigma) \tau_{\sigma}$. Because $(\varphi \sigma) \tau_{s} \subseteq \mathcal{D}_{0}$ and $\perp \in \mathcal{D}_{r}$, it follows that $\operatorname{supp}\left(\rho_{\perp}\right) \subseteq \operatorname{supp}(\chi)$. Hence $\perp \in \operatorname{Sig}\left(\mathcal{M}^{\chi}\right)$, and consequently $s=\perp \rightarrow \square \in \mathcal{M}^{\chi}$.

Otherwise, if $\rho^{\prime}$ is a proof of $\gamma=\perp$ then, as before, $\rho^{\prime}$ must be a proof in $\mathcal{P}^{\chi} \cup(\varphi \sigma) \tau_{\sigma}$. Then, since $(\varphi \sigma) \tau_{s} \subseteq \mathcal{D}_{0}$ and $\perp \in \mathcal{D}_{r}$, we have $s^{\prime} \in \operatorname{supp}(\chi)$ and thus $s \in \mathcal{M}^{\chi}$. If $\rho^{\prime}$ is a proof of $\gamma=(Q(\mathbf{x}) \sigma) \tau_{\sigma}$, let $\rho^{\prime}=(T, \lambda)$ with $v$ the root of $T$ and $w_{1}, \ldots, w_{m}$ its children. The rule applied at the top of $\rho^{\prime}$ must be from $\mathcal{O}_{Q}$ and therefore different from $s^{\prime}$. In particular, this rule must be of the form $\psi_{i}(\mathbf{x}, \mathbf{y}) \rightarrow Q(\mathbf{x})$, with $\left(\left(\psi_{i} \tilde{\sigma}\right) \tau_{\sigma}\right)=\bigwedge_{j=1}^{m} \lambda\left(w_{j}\right)$ for some extension $\tilde{\sigma}$ of $\sigma$ to $\mathbf{y}$. Clearly, there is some substitution $\sigma^{\prime}$ with domain $\mathbf{y}$ such that $\left(\left(\psi_{i} \tilde{\sigma}\right) \tau_{\sigma}\right)=\left(\left(\psi_{i} \sigma\right) \tau_{\sigma}\right) \sigma^{\prime}$. The rule $s^{\prime}$ must thus be in the support of a proof $\rho_{j}^{\prime}$ in $\mathcal{P}^{\chi} Q \cup(\varphi \sigma) \tau_{s}$ of some $\lambda\left(w_{j}\right)$. Since $r$ does not mention $Q$ neither does $\lambda\left(w_{j}\right)$, and again we have that $\rho^{\prime}$ must in fact be a proof in $\mathcal{P}^{\chi} \cup(\varphi \sigma) \tau_{\sigma}$. This implies $\mathcal{P}^{\chi} \cup(\varphi \sigma) \tau_{s}=\lambda\left(w_{j}\right)$ and hence by assumption $\lambda\left(w_{j}\right) \in \mathcal{D}_{r}$. Finally, since $(\varphi \sigma) \tau_{\sigma} \subseteq \mathcal{D}_{0}$, we have $s^{\prime} \in \operatorname{supp}(\chi)$ and consequently $s \in \mathcal{M}^{\chi}$.

\section{Modules for each Inseparability Relation}

Theorem 23 tells us how to choose a module setting $\chi$ so that its corresponding module $\mathcal{M}^{\chi}$ preserves a particular consequence of $\mathcal{O}$. However, in order for $\mathcal{M}^{\chi}$ to be a $\equiv_{\Sigma}^{\mathcal{S}}$-module of $\mathcal{O}$ for a given inseparability relation $\mathcal{S}$, it must preserve not one, but all of the (possibly infinitely many) relevant consequences in $\operatorname{rel}_{\mathcal{S}}(\mathcal{O}, \Sigma)$ (recall Theorem 9 in Section 3).

In this section we consider each inseparability relation $\mathcal{S} \in\{\mathrm{m}, \mathrm{q}, \mathrm{f}, \mathrm{i}\}$, and formulate a specific module setting $\chi_{\mathcal{S}}$ which provably yields a $\equiv_{\Sigma}^{\mathcal{S}}$-module of $\mathcal{O}$. Later on in Section 11 we will consider the optimality of these module settings - that is, whether there may exist a different setting that yields a smaller module for the relevant inseparability relation.

\subsection{Implication Inseparability}

Our running example immediately suggests a natural module setting $\chi_{\mathrm{i}}=\left\langle\theta^{\mathrm{i}}, \mathcal{D}_{0}^{\mathrm{i}}, \mathcal{D}_{r}^{\mathrm{i}}\right\rangle$ that guarantees implication inseparability. 
As in our example, we pick the substitution $\theta^{i}$ to be as "general" as possible by Skolemising each existentially quantified variable to a distinct fresh constant and mapping constants occurring in $\mathcal{O}$ to themselves. To pick $\mathcal{D}_{0}^{\mathrm{i}}$ and $\mathcal{D}_{r}^{\mathrm{i}}$ we rely on the application of the sufficient conditions established in Theorem 23 to each of the (quadratically many) $\Sigma$-implications $A(\mathbf{x}) \rightarrow B(\mathbf{x})$ with $\mathbf{x}=\left(x_{1}, \ldots, x_{n}\right)$. More precisely, to capture the instantiations of the body $A(\mathbf{x})$ we define $\mathcal{D}_{0}$ to contain a fact $A\left(c_{A}^{1}, \ldots, c_{A}^{n}\right)$ involving fresh constants $c_{A}^{i}$ uniquely associated to the predicate $A$; furthermore, to capture the head of the implication, we define $\mathcal{D}_{r}$ so as to contain a fact $B\left(c_{A}^{1}, \ldots, c_{A}^{n}\right)$. In this way, the dataset $\mathcal{D}_{0}^{\mathrm{i}}$ contains linearly many and $\mathcal{D}_{r}^{\mathrm{i}}$ quadratically many facts in the size of the signature $\Sigma$.

Definition 24. For each existentially quantified variable $y$ in $\mathcal{O}$, let $c_{y}$ be a fresh constant. Furthermore, for each $A \in \Sigma$ of arity $n$, let $\mathbf{c}_{A}=\left(c_{A}^{1}, \ldots, c_{A}^{n}\right)$ be an array of fresh constants. The module setting $\chi_{\mathrm{i}}=\left\langle\theta^{\mathrm{i}}, \mathcal{D}_{0}^{\mathrm{i}}, \mathcal{D}_{r}^{\mathrm{i}}\right\rangle$ is defined as follows:

- $\theta^{\mathrm{i}}=\left\{y \mapsto c_{y} \mid y\right.$ existentially quantified in $\left.\mathcal{O}\right\} \cup\{c \mapsto c \mid c \in \operatorname{Ct}(\mathcal{O})\}$,

- $\mathcal{D}_{0}^{\mathrm{i}}=\left\{A\left(\mathbf{c}_{A}\right) \mid A \in \Sigma\right\}$; and

- $\mathcal{D}_{r}^{\mathrm{i}}=\left\{B\left(\mathbf{c}_{A}\right) \mid A \neq B\right.$ predicates in $\Sigma$ of the same arity $\} \cup\{\perp\}$.

The module setting $\chi_{\mathrm{i}}$ is reminiscent of the datalog encodings typically used to check whether a concept $A$ is subsumed by another concept $B$ w.r.t. a "lightweight" ontology $\mathcal{O}$ (Krötzsch et al., 2008b; Stefanoni et al., 2013). There, existentially quantified variables in rules are also skolemised as fresh constants to produce a datalog program $\mathcal{P}$, and then it is checked whether $\mathcal{P} \cup\{A(a)\} \models B(a)$.

The module setting $\chi_{i}$ captures implication inseparability as a straightforward consequence of Theorem 23.

Theorem 25. $\mathcal{M}^{\chi_{i}} \equiv_{\Sigma}^{i} \mathcal{O}$.

Proof. Consider an arbitrary rule of the form $A(\mathbf{x}) \rightarrow B(\mathbf{x})$ with $\mathbf{x}=\left(x_{1}, \ldots, x_{n}\right)$ a vector of distinct variables and $A, B$ distinct $n$-ary predicates from $\Sigma$. Let $\sigma$ be a substitution mapping $x_{1}, \ldots, x_{n}$ to distinct constants $c_{1}, \ldots, c_{n}$, and $\tau_{\sigma}$ another substitution such that $c_{i} \tau_{\sigma}=c_{A}^{i}$. By definition of $\chi_{\mathrm{i}}$ we have $(A(\mathbf{x}) \sigma) \tau_{\sigma} \in \mathcal{D}_{0}^{\mathrm{i}}$ and $(B(\mathbf{x}) \sigma) \tau_{\sigma} \in \mathcal{D}_{r}^{\mathrm{i}}$, and thus, by Theorem 23, it follows that $\mathcal{O}=A(\mathbf{x}) \rightarrow B(\mathbf{x})$ iff $\mathcal{M}^{\chi_{i}}=A(\mathbf{x}) \rightarrow B(\mathbf{x})$.

\subsection{Fact Inseparability}

By Theorem 9, fact inseparability requires the preservation of all the datalog $\Sigma$-rules entailed by $\mathcal{O}$. Thus, in contrast to implication inseparability, it may require the preservation of a very large (and possibly even infinite) set of entailments. Unsurprisingly, the module setting $\chi_{\mathrm{i}}$ cannot be used to capture fact inseparability as illustrated by the following example.

Example 26. Consider $\mathcal{O}^{e x}$ and $\Sigma_{1}=\{B, C, D, H\}$, for which $\mathcal{M}^{\chi_{i}}=\left\{r_{5}-r_{8}\right\}$. As seen in Example 18, for $\mathcal{D}=\{B(a), C(a)\}$ it is the case that $\mathcal{O}^{e x} \cup \mathcal{D} \models D(a)$, while we also have $\mathcal{M}^{\chi_{\mathrm{i}}} \cup \mathcal{D} \not \models D(a)$; hence, $\mathcal{M}^{\chi_{\mathrm{i}}}$ is not $\Sigma_{1}$-fact inseparable from $\mathcal{O}^{e x}$.

Equivalently, $\mathcal{O}^{e x}$ entails the datalog rule $r_{3}=B(x) \wedge C(x) \rightarrow D(x)$, whereas $\mathcal{M}^{\chi_{i}}$ does not and hence Theorem 23 is no longer applicable since $\mathcal{D}$, which instantiates the body of $r_{3}$, cannot be embedded into $\mathcal{D}_{0}^{\mathrm{i}}=\left\{B\left(c_{B}\right), C\left(c_{C}\right), D\left(c_{D}\right), H\left(c_{H}\right)\right\}$. 
Thus, we will next define a suitable module setting $\chi_{\mathrm{f}}=\left\langle\theta^{\mathrm{f}}, \mathcal{D}_{0}^{\mathrm{f}}, \mathcal{D}_{r}^{\mathrm{f}}\right\rangle$ to capture fact inseparability. As in the previous case, we will exploit the sufficient conditions given in Theorem 23. To this end, we first need to make sure that $\mathcal{D}_{0}^{\mathrm{f}}\left(\right.$ resp. $\mathcal{D}_{r}^{\mathrm{f}}$ ) captures all possible body (resp. head) instantiations of all possible datalog rules over $\Sigma$ that may be entailed by $\mathcal{O}$. We achieve this by choosing $\mathcal{D}_{0}^{\mathrm{f}}$ and $\mathcal{D}_{r}^{\mathrm{f}}$ to be the "most constrained" $\Sigma$-dataset possible, which is typically referred to in the literature as the critical dataset (Marnette, 2009; Cuenca Grau, Horrocks, Krötzsch, Kupke, Magka, Motik, \& Wang, 2013).

Definition 27. Let $\Sigma$ be a signature and let $*$ be a fresh constant. The critical $\Sigma$-dataset is defined as follows:

$$
\mathcal{D}_{\Sigma}^{*}=\{A(\overbrace{*, \ldots, *}^{n}) \mid A n \text {-ary predicate in } \Sigma\}
$$

Indeed, it is straightforward to see that every $\Sigma$-dataset (and hence any datalog rule instantiation) can be embedded into $\mathcal{D}_{\Sigma}^{*}$ by mapping every constant into $*$.

As in the case of $\chi_{i}$, we choose the substitution $\theta^{f}$ to be as general as possible by mapping existentially quantified variables to distinct fresh constants. However, in contrast to $\chi_{i}$, we require all constants occurring in $\mathcal{O}$ to be mapped to $*$ rather than to themselves. This choice is justified by the following example.

Example 28. Consider $\mathcal{O}^{e x}$ and $\Sigma=\{A, C, E\}$. Clearly, for $\mathcal{D}=\{A(a), C(o)\}$ we have $\mathcal{O}^{e x} \cup \mathcal{D} \models E(a)$ due to rules $r_{2}$ and $r_{4}$ in $\mathcal{O}^{e x}$. If we were to pick $\theta^{\mathrm{f}}$ to be $\theta^{\mathrm{i}}$, which maps constant $o$ in $\mathcal{O}^{e x}$ to itself, we would obtain $\mathcal{M}^{\chi_{\mathrm{f}}}=\emptyset$ even if we choose $\mathcal{D}_{0}^{\mathrm{f}}$ and $\mathcal{D}_{r}^{\mathrm{f}}$ as the critical $\Sigma$-dataset. Indeed, the relevant fact $E(*)$ would not be provable from $\mathcal{P} \chi_{\mathrm{f}} \cup \mathcal{D}_{0}^{f}$. $\diamond$

We are now ready to define $\chi_{\mathrm{f}}$ formally.

Definition 29. Let constants $c_{y}$ be as in Definition 24, and let $*$ be a fresh constant. The module setting $\chi_{\mathrm{f}}=\left\langle\theta^{\mathrm{f}}, \mathcal{D}_{0}^{\mathrm{f}}, \mathcal{D}_{r}^{\mathrm{f}}\right\rangle$ is defined as follows:

- $\theta^{\mathrm{f}}=\left\{y \mapsto c_{y} \mid y\right.$ existentially quantified in $\left.\mathcal{O}\right\} \cup\{c \mapsto * \mid c \in \operatorname{Ct}(\mathcal{O})\}$,

- $\mathcal{D}_{0}^{f}=\mathcal{D}_{\Sigma}^{*}$, and

- $\mathcal{D}_{r}^{\mathrm{f}}=\mathcal{D}_{\Sigma}^{*} \cup\{\perp\}$.

Example 30. The datalog program generated by $\theta^{f}$ for $\mathcal{O}^{e x}$ coincides with that of Figure 5 in all rules except for $r_{2}$, which now becomes

$$
r_{2}^{\prime}: A(x) \rightarrow R(x, *)
$$

If we consider again $\Sigma_{1}=\{B, C, D, H\}$ and $\mathcal{D}=\{B(a), C(a)\}$ from Example 26, we clearly have $\mathcal{P} \chi_{\mathrm{f}} \cup \mathcal{D}_{0}^{\mathrm{f}} \vdash D(*) \in \mathcal{D}_{r}^{\mathrm{f}}$ since $\{B(*), C(*)\} \subseteq \mathcal{D}_{0}^{\mathrm{f}}$. The unique proof $\rho$ of $D(*)$ in $\mathcal{P} \chi_{\mathrm{f}} \cup \mathcal{D}_{0}^{\mathrm{f}}$ is only supported by $r_{3}$; this guarantees that $r_{3} \in \mathcal{M}^{\chi_{\mathrm{f}}}$ and thus $\rho$ corresponds directly to a proof of $D(a)$ in $\mathcal{M}^{\chi_{\mathrm{f}}} \cup \mathcal{D}$. Hence, we have $\mathcal{M}^{\chi_{\mathrm{f}}} \cup \mathcal{D}=D(a)$, as required.

If we now consider the signature $\Sigma=\{A, C, E\}$ from Example 28, we can observe in Figure 6 how our choice of mapping constant $o$ to $*$ ensures that the module contains the necessary rules $r_{2}$ and $r_{4}$. 


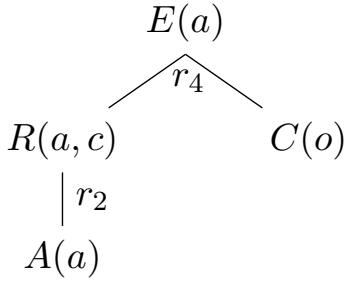

(a)

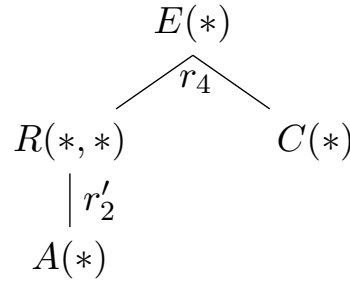

(b)

Figure 6: Proofs of (a) $E(a)$ in $\mathcal{O}^{e x} \cup\{A(a), C(o)\}$ and (b) $E(*)$ in $\mathcal{P}^{\chi_{\mathrm{f}}} \cup \mathcal{D}_{0}^{\mathrm{f}}$

We can now exploit Theorem 23 once more to show that $\chi_{\mathrm{f}}$ captures fact inseparability.

Theorem 31. $\mathcal{M}^{\chi_{\mathrm{f}}} \equiv_{\Sigma}^{\mathrm{f}} \mathcal{O}$.

Proof. Let $r=\varphi \rightarrow \gamma$ be a datalog rule over $\Sigma$. Let $\sigma$ be a substitution mapping all variables in $r$ to pairwise distinct constants, and $\tau_{*}$ a substitution mapping each constant in the range of $\sigma$ to $*$. By definition of $\chi_{\mathrm{f}}$ we have $(\varphi \sigma) \tau_{*} \subseteq \mathcal{D}_{0}^{\mathrm{f}}$ and $(\gamma \sigma) \tau_{*} \subseteq \mathcal{D}_{r}^{\mathrm{f}}$ and thus, by Theorem 23 it follows that $\mathcal{O}=r$ iff $\mathcal{M}^{\chi_{\mathrm{f}}}=r$. Finally, by Proposition 7 , this implies $\mathcal{M}^{\chi_{\mathrm{f}}} \equiv_{\Sigma}^{\mathrm{f}} \mathcal{O}$.

\subsection{Query Inseparability}

Positive existential queries constitute a much richer query language than facts as they allow for existentially quantified variables. Thus, the query inseparability requirement inevitably leads to larger modules.

Example 32. Consider $\mathcal{O}^{e x}$ and $\Sigma=\{A, B\}$. Given the $\Sigma$-dataset $\mathcal{D}=\{A(a)\}$ and the $\Sigma$-query $q=\exists y B(y)$, we have that $\mathcal{O}^{e x} \cup \mathcal{D} \models q$ (due to rule $r_{1}$ ). In this case, however, $\mathcal{M}^{\chi_{\mathrm{f}}}$ is empty and thus $\mathcal{M}^{\chi_{\mathrm{f}}} \cup \mathcal{D} \not \neq q$. Indeed, the only additional facts in the materialisation of $\mathcal{P}^{\chi_{\mathrm{f}}} \cup\{A(*), B(*)\}$ are $R\left(*, c_{y_{1}}\right)$ and $B\left(c_{y_{1}}\right)$, and hence neither $r_{1}^{\prime}$ nor $r_{1}^{\prime \prime}$ (cf. Figure 5) are in $\operatorname{supp}\left(\chi_{\mathrm{f}}\right)$. This suggests that, although the critical $\Sigma$-dataset $\mathcal{D}_{\Sigma}^{*}$ is constrained enough to embed every $\Sigma$-dataset, we may need to consider additional relevant facts to capture all proofs of all $\Sigma$-queries. In particular, rule $r_{1}$ implies that $B$ has a non-empty extension whenever $A$ does: a dependency that is then checked by $q$. This can be captured by considering fact $B\left(c_{y_{1}}\right)$ as relevant, in which case $r_{1}$ would be included in the module. $\diamond$

By Theorem 9, query inseparability requires the preservation of all $\Sigma$-rules entailed by $\mathcal{O}$ (and not just of those that are datalog). In particular, first-order rules may involve existentially quantified variables, which correspond in our framework to Skolem constants. This naturally suggests a module setting $\chi_{\mathrm{q}}$ that differs from $\chi_{\mathrm{f}}$ only in that $\Sigma$-facts involving Skolem constants (and not just those mentioning only $*$ ) are also considered relevant.

Definition 33. Let constants $c_{y}$ and $*$ be as in Definition 29. We define the module setting $\chi_{\mathrm{q}}=\left\langle\theta^{\mathrm{q}}, \mathcal{D}_{0}^{\mathrm{q}}, \mathcal{D}_{r}^{\mathrm{q}}\right\rangle$ as follows:

- $\theta^{\mathrm{q}}=\theta^{\mathrm{f}}$, 


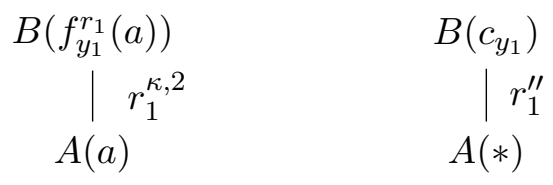

(a)

Figure 7: Proofs of (a) $B\left(f_{y_{1}}^{r_{1}}(a)\right)$ in $\mathcal{O}^{e x} \cup\{A(a)\}$ and (b) $B\left(c_{y_{1}}\right)$ in $\mathcal{P}^{\chi_{\mathfrak{q}}} \cup \mathcal{D}_{0}^{\mathrm{q}}$

- $\mathcal{D}_{0}^{\mathrm{q}}=\mathcal{D}_{\Sigma}^{*}$, and

- $\mathcal{D}_{r}^{\mathrm{q}}=\left\{A\left(a_{1}, \ldots, a_{n}\right) \mid A \in \Sigma\right.$, each $a_{j}$ is either $*$ or some $\left.c_{y}\right\} \cup\{\perp\}$.

Example 34. Coming back to Example 32, we can observe in Figure 7 how a proof of $B\left(f_{y_{1}}^{r_{1}}(a)\right)$ in $\kappa\left(\mathcal{O}^{e x}\right) \cup\{A(a)\}$ that is supported by $r_{1}^{\kappa, 2}: A(x) \rightarrow B\left(f_{y_{1}}^{r_{1}}(x)\right)$ can be recovered from a proof of $B\left(c_{y_{1}}\right)$ in $\mathcal{P} \chi_{\mathfrak{q}} \cup \mathcal{D}_{0}^{\mathrm{q}}$ that is supported by $r_{1}^{\prime \prime}$. The definition of $\chi_{\mathrm{q}}$ ensures that $B\left(c_{y_{1}}\right) \in \mathcal{D}_{r}^{q}$, and hence $r_{1} \in \mathcal{M}^{\chi_{q}}$.

Theorem 35. $\mathcal{M}^{\chi_{q}} \equiv_{\Sigma}^{q} \mathcal{O}$.

Proof. Let $r=\varphi \rightarrow \exists \mathbf{y} \psi$ be a rule over $\Sigma$ with a non-empty head. Let $\sigma$ be a substitution mapping all variables in $r$ to pairwise distinct constants and $\tau_{*}$ a substitution that maps each constant in the range of $\sigma$ to $*$. By definition of $\chi_{\mathrm{q}}$ we have $(\varphi \sigma) \tau_{*} \subseteq \mathcal{D}_{0}^{\mathrm{q}}$, and also $\psi \sigma^{\prime} \subseteq \mathcal{D}_{r}^{q}$ for each substitution $\sigma^{\prime}$ mapping all variables in $r$ to constants in $\operatorname{Ct}\left(\mathcal{D}_{0}^{\mathrm{q}} \cup \mathcal{D}_{r}^{\mathrm{q}}\right) \cup \operatorname{range}\left(\theta^{\mathrm{q}}\right)$. Thus, by Theorem 23, it follows that $\mathcal{O} \models r$ iff $\mathcal{M}^{\chi_{\mathrm{a}}} \models r$. By Proposition 7, this implies $\mathcal{M}^{\chi \mathrm{q}} \equiv{ }_{\Sigma}^{\mathrm{q}} \mathcal{O}$.

\subsection{Model Inseparability}

Model inseparability differs substantially from all the previous inseparability relations: rather than just the preservation of rule-shaped consequences, it requires the preservation of models (and hence of second-order consequences). Theorem 23, which we have repeatedly exploited to show that our modules preserve the required entailments, relies on the properties of hyperresolution (a first-order logic calculus); hence, it is not applicable to show preservation of second-order logic consequences. In particular, as the following example illustrates, the modules generated by $\chi_{\mathbf{q}}$ may not be $\Sigma$-model inseparable from $\mathcal{O}$.

Example 36. Consider $\mathcal{O}^{e x}$ and $\Sigma=\{A, C, D, R\}$, in which case $\mathcal{M}^{\chi_{q}}=\left\{r_{1}, r_{2}\right\}$. As we saw in Example 6, the interpretation $\mathcal{I}$ where $\Delta^{\mathcal{I}}=\{a, o\}, A^{\mathcal{I}}=\{a\}, B^{\mathcal{I}}=C^{\mathcal{I}}=\{o\}$, $D^{\mathcal{I}}=\emptyset$ and $R^{\mathcal{I}}=\{(a, o)\}$ is a model of $\mathcal{M}^{\chi_{\mathrm{q}}}$; however, it can be readily checked that it cannot be extended to a model of $\mathcal{O}$ without reinterpreting $A, C, D$ or $R$.

Intuitively, when constructing a model of $\mathcal{O}$, fixing the interpretation of certain predicates restricts the ways in which the remaining predicates can be interpreted. These restrictions are obviously determined by the dependencies introduced by the rules in $\mathcal{O}$. To capture model inseparability, we need to ensure that $\mathcal{M}^{\chi}$ preserves all such relevant dependencies between predicates in $\Sigma$. For this, we pick $\theta$ and $\mathcal{D}_{0}$ in such a way that any model 


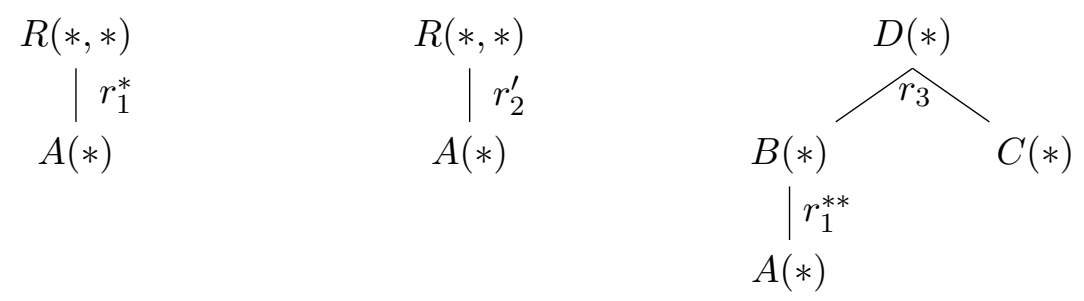

Figure 8: All proofs in $\mathcal{P}^{\chi_{\mathrm{m}}} \cup \mathcal{D}_{0}^{\mathrm{m}}$ of facts from $\mathcal{D}_{r}^{\mathrm{m}}$ in Example 39.

of $\mathcal{O}$ can be embedded in the materialisation of $\mathcal{P}^{\chi} \cup \mathcal{D}_{0}$; in turn, we choose $\mathcal{D}_{r}$ in such a way that it captures the $\Sigma$-reducts of all those models. If this is the case, the proofs in $\mathcal{P}^{\chi} \cup \mathcal{D}_{0}$ of facts from $\mathcal{D}_{r}$ will then capture all the dependencies between predicates in $\Sigma$.

Example 37. Note that $\mathcal{I}$ in Example 36 cannot be embedded in the materialisation of $\mathcal{P}^{\chi_{\mathrm{q}}} \cup \mathcal{D}_{0}^{\mathrm{q}}$ since the only facts over $\{B, C\}$ that it contains are $C(*)$ and $B\left(c_{y_{1}}\right)$, and the constant $o$ cannot be mapped to both $c_{y_{1}}$ and $*$.

To capture all models of $\mathcal{O}$, we once more pick $\mathcal{D}_{0}=\mathcal{D}_{\Sigma}^{*}$; but now, in contrast to $\chi_{\mathbf{q}}$, we choose a substitution $\theta$ that maps both existentially quantified variables and constants in $\mathcal{O}$ to $*$. Furthermore, to ensure that $\mathcal{D}_{r}$ captures the $\Sigma$-reducts of all those models, we pick $\mathcal{D}_{r}$ as $\mathcal{D}_{\Sigma}^{*}$ as well.

Definition 38. The module setting $\chi_{\mathrm{m}}=\left\langle\theta^{\mathrm{m}}, \mathcal{D}_{0}^{\mathrm{m}}, \mathcal{D}_{r}^{\mathrm{m}}\right\rangle$ is as follows:

- $\theta^{\mathrm{m}}=\{y \mapsto * \mid y$ existentially quantified in $\mathcal{O}\} \cup\{c \mapsto * \mid c \in \operatorname{Ct}(\mathcal{O})\}$,

- $\mathcal{D}_{0}^{\mathrm{m}}=\mathcal{D}_{\Sigma}^{*}$, and

- $\mathcal{D}_{r}^{\mathrm{m}}=\mathcal{D}_{\Sigma}^{*} \cup\{\perp\}$.

Example 39. Consider $\mathcal{O}^{e x}, \Sigma$ and $\mathcal{I}$ as in Example 36. The substitution $\theta^{\mathrm{m}}$ maps the existentially quantified variables in $r_{1}$ and $r_{6}$ to $*$. Thus, rules $r_{1}$ and $r_{6}$ in $\mathcal{O}^{\text {ex }}$ correspond to the following rules in $\mathcal{P}^{\chi_{m}}$ :

$$
\begin{aligned}
& r_{1} \rightsquigarrow r_{1}^{*}: \quad A(x) \rightarrow R(x, *), \quad r_{1}^{* *}: \quad A(x) \rightarrow B(*) \\
& r_{6} \rightsquigarrow r_{6}^{*}: F(x) \rightarrow S(x, *)
\end{aligned}
$$

The materialisation of $\mathcal{P}^{\chi_{\mathrm{m}}} \cup \mathcal{D}_{0}^{\mathrm{m}}$ contains facts $A(*), C(*), D(*), R(*, *)$, and $B(*)$. Consequently, it is now possible to embed the interpretation $\mathcal{I}$ into the aforementioned materialisation by mapping both $a$ and $o$ to $*$.

Figure 8 shows all (non-trivial) proofs in $\mathcal{P}^{\chi_{\mathrm{m}}} \cup \mathcal{D}_{0}^{\mathrm{m}}$ of facts from $\mathcal{D}_{r}^{\mathrm{m}}$. We can observe that the module $\mathcal{M}^{\chi_{\mathrm{m}}}$ consists of rules $r_{1}-r_{3}$. Clearly, any model of $\mathcal{M}^{\chi_{\mathrm{m}}}$ can be extended to a model of $\mathcal{O}^{e x}$ since no predicates from $\mathcal{M}^{\chi_{\mathrm{m}}}$ occur in the head of any rule in $\mathcal{O}^{e x} \backslash \mathcal{M}^{\chi_{\mathrm{m}}}$. $\diamond$

Theorem 40 shows that the module $\mathcal{M}^{\chi_{\mathrm{m}}}$ is $\Sigma$-model inseparable from $\mathcal{O}$. Indeed, every model $\mathcal{I}$ of $\mathcal{M}^{\chi_{\mathrm{m}}}$ can be extended to a model of $\mathcal{O}$ in the following way: (i) predicates not occurring in the materialisation of $\mathcal{P}^{\chi_{\mathrm{m}}} \cup \mathcal{D}_{0}^{\mathrm{m}}$ are interpreted as empty, (ii) predicates in the support of $\chi_{\mathrm{m}}$ (and hence occurring in $\mathcal{M}^{\chi_{\mathrm{m}}}$ ) are interpreted as in $\mathcal{I}$, and (iii) all other predicates $A$ with arity $n$ are interpreted as $\left(\Delta^{\mathcal{I}}\right)^{n}$. 
Theorem 40. $\mathcal{M}^{\chi_{\mathrm{m}}} \equiv \sum_{\Sigma}^{\mathrm{m}} \mathcal{O}$.

Proof. Let $\mathcal{I}$ be a model of $\mathcal{M}^{\text {女m }}$. W.l.o.g., we assume that $\mathcal{I}$ is defined over all of $\operatorname{Sig}(\mathcal{O})$. Let $\mathcal{J}$ be an interpretation with the same domain $\Delta$ as $\mathcal{I}$, and such that

$$
A^{\mathcal{J}}= \begin{cases}A^{\mathcal{I}} & \text { if } A \in \Sigma \cup \operatorname{Sig}\left(\operatorname{supp}\left(\chi_{\mathrm{m}}\right)\right) \\ \Delta^{\operatorname{arity}(A)} & \text { if } A \in \operatorname{Sig}\left(\mathcal{P}^{\chi_{\mathrm{m}}}\left(\mathcal{D}_{0}^{\mathrm{m}}\right)\right) \backslash\left(\Sigma \cup \operatorname{Sig}\left(\operatorname{supp}\left(\chi_{\mathrm{m}}\right)\right)\right) \\ \emptyset & \text { otherwise }\end{cases}
$$

Note that $\Sigma \cup \operatorname{Sig}\left(\operatorname{supp}\left(\chi_{\mathrm{m}}\right)\right) \subseteq \operatorname{Sig}\left(\mathcal{P} \chi_{\mathrm{m}}\left(\mathcal{D}_{0}^{\mathrm{m}}\right)\right)$.

Consider $r: \varphi \rightarrow \psi \in \mathcal{O}$. We now show that $\mathcal{J} \models r$.

Assume first $\psi=\square$. Then $r=\perp \rightarrow \square$ and we need to check that $\perp$ is interpreted as false. If $\perp \notin \operatorname{Sig}\left(\mathcal{P}^{\chi_{\mathrm{m}}}\left(\mathcal{D}_{0}^{\mathrm{m}}\right)\right)$ then this is the case by definition of $\mathcal{J}$. If $\perp \in \operatorname{Sig}\left(\mathcal{P}^{\chi_{\mathrm{m}}}\left(\mathcal{D}_{0}^{\mathrm{m}}\right)\right)$ then, since $\perp \in \mathcal{D}_{r}^{\mathrm{m}}$, it must be $\perp \in \operatorname{Sig}\left(\operatorname{supp}\left(\chi_{\mathrm{m}}\right)\right)$ and therefore also $\perp \in \operatorname{Sig}\left(\mathcal{M}^{\chi_{\mathrm{m}}}\right)$. This implies that $\perp \rightarrow \square \in \mathcal{M}^{\chi_{\mathrm{m}}}$ and thus, since $\mathcal{I}$ is a model of $\mathcal{M}^{\chi_{\mathrm{m}}}$, it must be the case that $\perp$ is interpreted as false. Since $\perp \in \operatorname{Sig}\left(\operatorname{supp}\left(\chi_{\mathrm{m}}\right)\right)$, by definition of $\mathcal{J}$ we then have that both $\perp^{\mathcal{J}}$ and $\perp^{\mathcal{I}}$ are false.

Assume now $\psi \neq \square$. If $\operatorname{Sig}(\psi) \nsubseteq \operatorname{Sig}\left(\mathcal{P}^{\chi_{\mathrm{m}}}\left(\mathcal{D}_{0}^{\mathrm{m}}\right)\right)$ then, because $\mathcal{P}^{\chi_{\mathrm{m}}} \cup \mathcal{D}_{0}^{\mathrm{m}}$ only mentions one constant (namely, *), it follows that also $\operatorname{Sig}(\varphi) \nsubseteq \operatorname{Sig}\left(\mathcal{P}^{\chi_{\mathrm{m}}}\left(\mathcal{D}_{0}^{\mathrm{m}}\right)\right)$ and therefore $\varphi^{\mathcal{J}}=\emptyset$ and $\mathcal{J}=r$. Hence, in the following we assume $\operatorname{Sig}(\psi) \subseteq \operatorname{Sig}\left(\mathcal{P}^{\chi_{\mathrm{m}}}\left(\mathcal{D}_{0}^{\mathrm{m}}\right)\right)$.

If $\operatorname{Sig}(\psi) \cap\left(\Sigma \cup \operatorname{Sig}\left(\operatorname{supp}\left(\chi_{\mathrm{m}}\right)\right)\right)=\emptyset$, then $A^{\mathcal{J}}=\Delta^{\text {arity }(A)}$ for each $A \in \operatorname{Sig}(\psi)$ and it is immediate that $\mathcal{J} \models r$. Otherwise suppose that there exists a substitution $\sigma$ over all variables in $r$ such that $\mathcal{J}=\varphi \sigma$ (if no such substitution exists then $\mathcal{J}=r$ holds trivially). Then $\varphi^{\mathcal{J}} \neq \emptyset$ and it must be $\operatorname{Sig}(\varphi) \subseteq \operatorname{Sig}\left(\mathcal{P}^{\chi_{\mathrm{m}}}\left(\mathcal{D}_{0}^{\mathrm{m}}\right)\right)$. Let $\sigma_{*}$ be a substitution that maps all variables to $*$; because $*$ is the only constant in $\mathcal{P} \chi_{\mathrm{m}}^{\mathrm{m}} \cup \mathcal{D}_{0}^{\mathrm{m}}$, it folows that $\varphi \sigma_{*} \subseteq \mathcal{P}^{\chi_{\mathrm{m}}}\left(\mathcal{D}_{0}^{\mathrm{m}}\right)$ and thus also $\psi \sigma_{*} \subseteq \mathcal{P}^{\chi_{\mathrm{m}}}\left(\mathcal{D}_{0}^{\mathrm{m}}\right)$.

By assumption, there exists $\gamma=A(*, \ldots, *) \in \psi \sigma_{*}$ with $A \in \Sigma \cup \operatorname{Sig}\left(\operatorname{supp}\left(\chi_{\mathrm{m}}\right)\right)$. Because $\varphi \sigma_{*} \subseteq \mathcal{P}^{\chi_{\mathrm{m}}}\left(\mathcal{D}_{0}^{\mathrm{m}}\right)$, there is a proof $\rho_{\gamma, r}$ of $\gamma$ in $\mathcal{P}^{\chi_{\mathrm{m}}} \cup \mathcal{D}_{0}^{\mathrm{m}}$ that is supported by a rule from $\Xi^{\chi_{\mathrm{m}}}(r)$. If $A \in \Sigma$ then, by definition of $\chi_{\mathrm{m}}$, it is $\gamma \in \mathcal{D}_{r}^{\chi_{\mathrm{m}}}$ and consequently $r \in \mathcal{M}^{\chi_{\mathrm{m}}}$. If, on the other hand, $A \notin \Sigma$, there must be $\gamma^{\prime} \in \mathcal{D}_{r}^{\mathrm{m}}$ and a proof $\rho^{\prime}$ of $\gamma^{\prime}$ in $\mathcal{P} \chi_{\mathrm{m}} \cup \mathcal{D}_{0}^{\mathrm{m}}$ such that some proof of $A(*, \ldots, *)$ is a subproof of $\rho^{\prime}$. Replacing this subproof with $\rho_{A, r}$ results in another proof of $\gamma^{\prime}$ in $\mathcal{P}^{\chi_{\mathrm{m}}} \cup \mathcal{D}_{0}^{\mathrm{m}}$ that is supported by a rule in $\Xi^{\chi_{\mathrm{m}}}(r)$. Thus $r \in \mathcal{M}^{\chi_{\mathrm{m}}}$ in this case as well. Rules in $\Xi^{\chi_{\mathrm{m}}}(r)$ have the same body as $r$, so $r \in \mathcal{M}^{\chi_{\mathrm{m}}}$ implies $\operatorname{Sig}(\varphi) \subseteq \operatorname{Sig}\left(\operatorname{supp}\left(\chi_{\mathrm{m}}\right)\right)$, and thus $\mathcal{I}$ and $\mathcal{J}$ agree over $\operatorname{Sig}(\varphi)$. By assumption, $\mathcal{J} \models \varphi \sigma$, so we have $\mathcal{I}=\varphi \sigma$ as well, and, since $r \in \mathcal{M}^{\chi_{\mathrm{m}}}$, also $\mathcal{I}=\psi \sigma$. Finally, since $\psi \sigma_{*} \subseteq \mathcal{P}^{\chi_{\mathrm{m}}}\left(\mathcal{D}_{0}^{\mathrm{m}}\right)$, we have that $\operatorname{Sig}(\psi) \subseteq \operatorname{Sig}\left(\mathcal{P}^{\chi_{\mathrm{m}}}\left(\mathcal{D}_{0}^{\mathrm{m}}\right)\right)$ and therefore $\psi^{\mathcal{I}} \subseteq \psi^{\mathcal{J}}$, so $\mathcal{J} \models \psi \sigma$. Since $\sigma$ is arbitrary, we can conclude that $\mathcal{J} \models r$.

The modules generated by $\chi_{\mathrm{m}}$ are similar in spirit to locality-based modules in that certain symbols outside the signature of the module are interpreted as either the empty set or the universal relation (of the relevant arity) over the interpretation domain. As we will show later on, $\mathcal{M}^{\chi_{m}} \subseteq \mathcal{M}_{[\mathcal{O}, \Sigma]}^{\perp}$ whenever $\mathcal{O}$ is a normalised $\mathcal{S} \mathcal{R O} \mathcal{I} \mathcal{Q}$ ontology. However, as illustrated by the following example, our modules are incomparable to $T$ - and $\perp T^{*}$-modules.

Example 41. For $\mathcal{O}=\mathcal{O}^{e x}$ and $\Sigma=\{D, F\}$ we have the following:

$$
\mathcal{M}^{\chi_{\mathrm{m}}}=\left\{r_{5}\right\} \quad \mathcal{M}_{[\mathcal{O}, \Sigma]}^{\top}=\left\{r_{1}-r_{3}\right\} \quad \mathcal{M}_{[\mathcal{O}, \Sigma]}^{\perp \top^{*}}=\emptyset .
$$


Consequently, $\mathcal{M}^{\chi_{\mathrm{m}}}$ is neither contained in $\mathcal{M}_{[\mathcal{O}, \Sigma]}^{\top}$, nor in $\mathcal{M}_{[\mathcal{O}, \Sigma]}^{\perp \top^{*}}$. To see that the converse is also true, consider $\mathcal{O}=\left\{r_{1}, r_{9}\right\}$, with $r_{9}=C(x) \rightarrow B(x)$, and $\Sigma=\{A, C, R\}$. Then, we have the following:

$$
\mathcal{M}^{\chi_{m}}=\left\{r_{1}\right\} \quad \mathcal{M}_{[\mathcal{O}, \Sigma]}^{\top}=\mathcal{M}_{[\mathcal{O}, \Sigma]}^{\perp \top^{*}}=\mathcal{O}
$$

We have already mentioned that modules generated by $\chi_{m}$ are included in locality $\perp$-modules. To conclude this section, we show how $\chi_{\mathrm{m}}$ can be modified to precisely capture $\perp$-modules. For this, it suffices to modify $\chi_{\mathrm{m}}$ by making $\mathcal{D}_{r}$ the critical dataset over the entire signature of $\mathcal{O}$ (instead of just $\Sigma$ ) as given in the following definition.

Definition 42. The module setting $\chi_{\mathrm{b}}=\left\langle\theta^{\mathrm{b}}, \mathcal{D}_{0}^{\mathrm{b}}, \mathcal{D}_{r}^{\mathrm{b}}\right\rangle$ is as follows:

- $\theta^{\mathrm{b}}=\theta^{\mathrm{m}}$,

- $\mathcal{D}_{0}^{\mathrm{b}}=\mathcal{D}_{\Sigma}^{*}$, and

- $\mathcal{D}_{r}^{\mathrm{b}}=\mathcal{D}_{\operatorname{Sig}(\mathcal{O})}^{*} \cup\{\perp\}$.

The following proposition shows that $\mathcal{M}^{\chi_{\mathrm{b}}}$ coincides with the $\perp$-locality module of a $\mathcal{S R O \mathcal { Q } Q}$ ontology $\mathcal{O}$ relative to $\Sigma$.

Proposition 43. If $\mathcal{O}$ is a normalised $\mathcal{S R O} \mathcal{I} \mathcal{Q}$ ontology, then $\mathcal{M}^{\chi_{\mathrm{b}}}=\mathcal{M}_{[\mathcal{O}, \Sigma]}^{\perp}$.

Proof. By definition, $\mathcal{M}_{[\mathcal{O}, \Sigma]}^{\perp}$ is the smallest subset $\mathcal{M} \subseteq \mathcal{O}$ such that every axiom in $\mathcal{O} \backslash \mathcal{M}$ is $\perp$-local w.r.t. $\Sigma \cup \operatorname{Sig}(\mathcal{M})$. To show that $\mathcal{M}_{[\mathcal{O}, \Sigma]}^{\perp} \subseteq \mathcal{M}^{\chi_{\mathrm{b}}}$, it suffices to show that, for each $r \in \mathcal{O} \backslash \mathcal{M}^{\chi_{\mathrm{b}}}, r$ is $\perp$-local w.r.t. $\Sigma \cup \operatorname{Sig}\left(\mathcal{M}^{\chi_{\mathrm{b}}}\right)$. Consider $r \in \mathcal{O} \backslash \mathcal{M}^{\chi_{\mathrm{b}}}$. Since $\mathcal{D}_{r}^{\mathrm{b}}$ contains all facts that can occur in $\mathcal{P} \chi_{\mathrm{b}}\left(\mathcal{D}_{0}^{\mathrm{b}}\right)$, we have that $\operatorname{supp}\left(\chi_{\mathrm{b}}\right)$ consists of all rules in the support of any proof in $\mathcal{P}^{\chi_{\mathrm{b}}} \cup \mathcal{D}_{0}^{\mathrm{b}}$. Furthermore, we have that $\operatorname{Sig}\left(\mathcal{M}^{\chi_{\mathrm{b}}}\right) \cup \Sigma=\operatorname{Sig}\left(\mathcal{P}^{\chi_{\mathrm{b}}}\left(\mathcal{D}_{0}^{\mathrm{b}}\right)\right)$. Therefore, there can be no proof in $\mathcal{P}^{\chi_{\mathrm{b}}} \cup \mathcal{D}_{0}^{\mathrm{b}}$ that has a rule from $\Xi^{\chi_{\mathrm{b}}}(r)$ in its support. Because the only constant mentioned in $\mathcal{P}^{\chi_{\mathrm{b}}} \cup \mathcal{D}_{0}^{\mathrm{b}}$ is $*$, this means that some predicate from the body of $r$ does not occur at all in $\operatorname{Sig}\left(\mathcal{P} \chi_{\mathrm{b}}\left(\mathcal{D}_{0}^{\mathrm{b}}\right)\right)=\operatorname{Sig}\left(\operatorname{supp}\left(\chi_{\mathrm{b}}\right)\right) \cup \Sigma$. As can be observed in Tables 1 and 2, this implies that $r$ is $\perp$-local w.r.t. $\operatorname{Sig}\left(\operatorname{supp}\left(\chi_{\mathbf{b}}\right)\right) \cup \Sigma$.

To see that $\mathcal{M}^{\chi_{\mathrm{b}}} \subseteq \mathcal{M}_{[\mathcal{O}, \Sigma]}^{\perp}$, consider $r \in \mathcal{M}^{\chi_{\mathrm{b}}}$. There must exist $r^{\prime} \in \Xi^{\chi_{\mathrm{b}}}(r)$ such that $r^{\prime} \in \operatorname{supp}(\rho)$ for some proof $\rho$ in $\mathcal{P}^{\chi_{\mathrm{b}}} \cup \mathcal{D}_{0}^{\mathrm{b}}$. To show that $r \in \mathcal{M}_{[\mathcal{O}, \Sigma]}^{\perp}$, let us reason by induction on the depth $d$ of $\rho$.

$d=0$ Then the body of $r$ is empty and thus $r$ is not $\perp$-local w.r.t. any signature. It follows that $r \in \mathcal{M}_{[\mathcal{O}, \Sigma]}^{\perp}$.

$d>0$ It suffices to consider the case where $r^{\prime}$ is the rule applied at the top of $\rho$, since we already know by induction hypothesis that, for each $s \in \mathcal{O}$ such that a rule from $\Xi^{\chi_{\mathrm{b}}}(s)$ is in the support of a (proper) subproof of $\rho$, it is $s \in \mathcal{M}_{[\mathcal{O}, \Sigma]}^{\perp}$. Since $\operatorname{Sig}\left(\mathcal{D}_{0}^{\mathrm{b}}\right)=\Sigma$, this implies that, if $\rho=(T, \lambda)$ with $v$ the root of $T$ and $w_{1}, \ldots, w_{n}$ its children, then $\operatorname{Sig}\left(\lambda\left(w_{i}\right)\right) \subseteq \operatorname{Sig}\left(\mathcal{M}_{[\mathcal{O}, \Sigma]}^{\perp}\right) \cup \Sigma$. Consequently, the body of $r$ must have its signature fully contained in $\operatorname{Sig}\left(\mathcal{M}_{[\mathcal{O}, \Sigma]}^{\perp}\right) \cup \Sigma$. It follows that $r$ is not $\perp$-local w.r.t. $\operatorname{Sig}\left(\mathcal{M}_{[\mathcal{O}, \Sigma]}^{\perp}\right) \cup \Sigma$ and hence $r \in \mathcal{M}_{[\mathcal{O}, \Sigma]}^{\perp}$. 


\section{Additional Inseparability Relations}

The bulk of the research on module extraction has focused on the inseparability relations considered in Section 6. In this section we show how our framework can be seamlessly adapted to other interesting inseparability relations.

\subsection{Classification Inseparability}

Classification - the problem of identifying all subsumption relationships between all pairs of atomic concepts and all pairs of atomic roles in a DL ontology - is a fundamental reasoning task in ontology engineering. Classifying a first-order ontology $\mathcal{O}$ amounts to computing, for each predicate $A$ in $\mathcal{O}$, all the entailed implications $A(\mathbf{x}) \rightarrow B(\mathbf{x})$ where $B$ is a predicate of the same arity as $A$, referred to as a subsumer of $A$ in $\mathcal{O}$.

Locality $\perp$-modules have been successfully exploited for optimising classification of DL ontologies (Tsarkov \& Palmisano, 2012; Cuenca Grau et al., 2010; Armas Romero et al., 2012). In addition to being model-inseparable from the given ontology and satisfying the properties in Proposition 15 from Section 3.2, $\perp$-modules enjoy an additional property that makes them well-suited for optimising classification (Cuenca Grau et al., 2007a, 2010):

Proposition 44. Let $\mathcal{O}$ be an ontology, $\Sigma$ a signature, and $r$ a rule of the form $A(\mathbf{x}) \rightarrow \psi$ where $A \in \Sigma$ and either $\psi=\perp$ or $\psi=B(\mathbf{x})$ with $B \in \operatorname{Sig}(\mathcal{O})$. Then, $\mathcal{O} \models r$ iff $\mathcal{M}_{[\mathcal{O}, \Sigma]}^{\perp}=r$.

It follows from Proposition 44 that the $\perp$-module for $\Sigma=\{A\}$ in $\mathcal{O}$ captures all subsumers of $A$ in $\mathcal{O}$, and hence it is indistinguishable from $\mathcal{O}$ w.r.t. to all implications having $A$ in the body. We can capture this additional property of $\perp$-modules by means of the following inseparability relation.

Definition 45. Ontologies $\mathcal{O}$ and $\mathcal{O}^{\prime}$ are $\Sigma$-classification inseparable $\left(\mathcal{O} \equiv_{\Sigma}^{c} \mathcal{O}^{\prime}\right)$ if for each rule $r$ of the form $A(\mathbf{x}) \rightarrow \psi$, where $A \in \Sigma$ and either $\psi=\square$ or $\psi=B(\mathbf{x})$ with $B \in \operatorname{Sig}\left(\mathcal{O} \cup \mathcal{O}^{\prime}\right)$, we have $\mathcal{O} \models r$ iff $\mathcal{O}^{\prime} \models r$. Furthermore, rel ${ }_{\mathrm{c}}$ is the function mapping each ontology $\mathcal{O}$ and signature $\Sigma$ to the following set of rules:

$$
\operatorname{rel}_{\mathrm{c}}(\mathcal{O}, \Sigma)=\{r=A(\mathbf{x}) \rightarrow \psi \mid \mathcal{O} \models r, A \in \Sigma \text { and } \psi=\perp \text { or } \psi=B(\mathbf{x}) \text { with } B \in \operatorname{Sig}(\mathcal{O})\} \diamond
$$

It follows straightforwardly from the definition that $\mathcal{O} \equiv_{\Sigma}^{c} \mathcal{O}^{\prime}$ holds iff $\operatorname{rel}_{c}(\mathcal{O}, \Sigma)$ coincides with $\operatorname{rel}_{c}\left(\mathcal{O}^{\prime}, \Sigma\right)$; hence, Theorem 9 in Section 3 trivially extends to classification inseparability. Furthermore, it can be readily checked that $\equiv_{\Sigma}^{c} \subsetneq \equiv_{\Sigma}^{\mathrm{i}}$ for each non-trivial signature $\Sigma$, and hence classification inseparability is (as expected) a stronger requirement than implication inseparability. Finally, although $\perp$-modules ensure classification inseparability from $\mathcal{O}$, they are also model-inseparable (a much stricter requirement) and, as shown in Section 13, can be much larger than necessary for ontology classification.

Example 46. Consider our example ontology $\mathcal{O}^{e x}$. We can observe how classification inseparability is a stronger requirement than implication inseparability by considering $\Sigma=\{G\}$. Clearly, $\mathcal{M}=\emptyset$ is $\Sigma$-implication inseparable from $\mathcal{O}^{e x}$, whereas $\Sigma$-classification inseparability requires rule $r_{8}$ to be contained in $\mathcal{M}$. To see how $\perp$-modules differ from minimial classification-inseparable modules consider $\Sigma=\{A\}$; in this case, we have that $\mathcal{M}_{\left[\mathcal{O}^{e x}, \Sigma\right]}^{\perp}=\left\{r_{1}, r_{2}\right\}$, but $A$ has no subsumers in $\mathcal{O}^{e x}$ and therefore the empty ontology is already $\Sigma$-classification inseparable from $\mathcal{O}^{e x}$. 
The following module setting extends $\chi_{i}$ from Definition 24 to capture classification inseparability. As one would naturally expect, the only required modification is to extend $\mathcal{D}_{r}^{\mathrm{i}}$ with facts involving predicates outside $\Sigma$.

Definition 47. For each $A \in \Sigma$ of arity $n$, let $\mathbf{c}_{A}=\left(c_{A}^{1}, \ldots, c_{A}^{n}\right)$ be an array of fresh constants. The module setting $\chi_{\mathrm{c}}=\left(\theta^{\mathrm{c}}, \mathcal{D}_{0}^{\mathrm{c}}, \mathcal{D}_{r}^{\mathrm{c}}\right)$ is defined as follows:

- $\theta^{\mathrm{c}}=\theta^{\mathrm{i}}$,

- $\mathcal{D}_{0}^{\mathrm{c}}=\mathcal{D}_{0}^{\mathrm{i}}$, and

- $\mathcal{D}_{r}^{c}=\left\{B\left(\mathbf{c}_{A}\right) \mid A \in \Sigma\right.$ and $B \in \operatorname{Sig}(\mathcal{O})$ distinct predicates of the same arity $\} \cup\{\perp\}$.

Example 48. Consider again $\mathcal{O}=\mathcal{O}^{e x}$ and $\Sigma=\{A\}$. Since no fact from $\mathcal{D}_{r}^{c}$ is provable in $\mathcal{P}^{\chi_{c}} \cup \mathcal{D}_{0}^{c}$ the module $\mathcal{M}^{\chi_{c}}$ is empty and thus also minimal in this case.

We can show that $\chi_{\mathrm{c}}$ captures implication inseparability using an argument analogous to that in the proof of Theorem 25 .

Theorem 49. $\mathcal{M}^{\chi c} \equiv_{\Sigma}^{c} \mathcal{O}$.

\subsection{Weak Query Inseparability}

One of the possible applications of modules based on query inseparability is to optimise query answering. In particular, if $\mathcal{M}$ is $\Sigma$-query inseparable from $\mathcal{O}$, then $\mathcal{O} \cup \mathcal{D}=q$ iff $\mathcal{M} \cup \mathcal{D}=q$ for any $\Sigma$-query $q$ and $\Sigma$-dataset $\mathcal{D}$; thus, we can replace $\mathcal{O}$ with $\mathcal{M}$ to answer an arbitrary query w.r.t. arbitrary data provided that only symbols in $\Sigma$ are deemed relevant.

While the aforementioned notion of query inseparability is useful for situations where the data is unknown or frequently changing, in many situations the data in an ontology is considered fixed and hence one could potentially extract smaller modules by not requiring $\mathcal{M}$ to be robust under extensions with arbitrary data.

Botoeva, Kontchakov, Ryzhikov, Wolter, and Zakharyaschev (2014) investigated a restricted notion of query inseparability that is well-suited for cases where the data in an ontology can be considered fixed. In this paper, we will refer to this restricted notion as weak query inseparability.

Definition 50. Ontologies $\mathcal{O}$ and $\mathcal{O}^{\prime}$ are $\Sigma$-weak query inseparable $\left(\mathcal{O} \equiv{ }_{\Sigma}^{\text {wq }} \mathcal{O}^{\prime}\right)$ if for each Boolean PEQ $q$ over $\Sigma$ we have $\mathcal{O} \models q$ iff $\mathcal{O}^{\prime} \models q$. Furthermore, rel wq is the function mapping each ontology $\mathcal{O}$ and signature $\Sigma$ to the set

$$
\text { rel }_{\text {wq }}(\mathcal{O}, \Sigma)=\{q \mid \mathcal{O} \models q \text { and } q \text { is a Boolean PEQ such that } \operatorname{Sig}(q) \subseteq \Sigma\} \quad \diamond
$$

Again, Theorem 9 extends naturally to weak query inseparability; indeed, $\mathcal{O} \equiv_{\Sigma}^{\text {wq }} \mathcal{O}^{\prime}$ if and only if $\operatorname{rel}_{w q}(\mathcal{O}, \Sigma)$ coincides with rel wq $\left(\mathcal{O}^{\prime}, \Sigma\right)$. Moreover, the requirements in Definition 50 are weaker than those of query inseparability and hence $\equiv_{\Sigma}^{q} \subsetneq \equiv_{\Sigma}^{\text {wq }}$ for each $\Sigma$. As a result, weak query inseparability may yield smaller modules, as we show in Section 13.

We next propose a module setting $\chi_{\text {wq }}$ that captures weak query inseparability. The main difference between $\chi_{\mathrm{wq}}$ and $\chi_{\mathrm{q}}$ in Section 6.3 is that the initial dataset $\mathcal{D}_{0}^{\mathrm{wq}}$ is chosen as 
empty rather than $\mathcal{D}_{\Sigma}^{*}$. This is a natural choice given that we no longer have the requirement that arbitrary $\Sigma$-datasets must be embeddable into $\mathcal{D}_{0}^{\mathrm{wq}}$. Furthermore, $\theta^{\mathrm{wq}}$ differs from $\theta^{\mathrm{q}}$ in that it maps constants from $\mathrm{Ct}(\mathcal{O})$ to themselves (same as $\theta^{\mathrm{i}}$ ).

Definition 51. Let constants $c_{y}$, for each existentially quantified variable $y$ in $\mathcal{O}$, be as in Definition 24. The module setting $\chi_{\mathrm{wq}}=\left\langle\theta^{\mathrm{wq}}, \mathcal{D}_{0}^{\mathrm{wq}}, \mathcal{D}_{r}^{\mathrm{wq}}\right\rangle$ is defined as follows:

- $\theta^{\mathrm{wq}}=\theta^{\mathrm{i}}$

- $\mathcal{D}_{0}^{\mathrm{wq}}=\emptyset$, and

- $\mathcal{D}_{r}^{\mathrm{wq}}=\left\{A\left(a_{1}, \ldots, a_{n}\right) \mid A \in \Sigma\right.$, each $a_{j}$ either in $\operatorname{Ct}(\mathcal{O})$ or equals some $\left.c_{y}\right\} \cup\{\perp\}$. $\diamond$

Example 52. Consider the extension of $\mathcal{O}^{e x}$ with the fact $\rightarrow D(i)$ (seen as a ground rule) and the signature $\Sigma=\{B, C, D, H\}$. It can be readily checked that $\mathcal{M}^{\chi_{a}}=\left\{r_{3}, r_{5}-r_{8}\right\}$, whereas $\mathcal{M}^{\chi_{\text {wq }}}=\left\{r_{5}-r_{8}\right\}$.

Theorem 53. $\mathcal{M}^{\chi_{w q}} \equiv_{\Sigma}^{w q} \mathcal{O}$.

Proof. Any Boolean PEQ $q$ can be seen as the rule $(\rightarrow q)$. Consequently, $\mathcal{M}^{\text {wq }} \equiv_{\Sigma}^{\text {wq }} \mathcal{O}$ follows from Theorem 23 by a similar argument to that in the proof of Theorem 35 .

\section{Module Containment}

Intuitively, the more expressive the language for which preservation of consequences is required, the larger the modules need to be. For instance, since $\equiv_{\Sigma}^{f} \subsetneq \equiv_{\Sigma}^{i}$, it is to be expected that the module $\mathcal{M}^{\chi_{\mathrm{i}}}$ obtained for implication inseparability is contained in the module $\mathcal{M}^{\chi_{\mathrm{f}}}$ for fact inseparability. We next show that all our modules in Sections 6 and 7 are consistent with this intuition.

Our first step will be to introduce a notion of homomorphism between module settings, which will then allow us to establish containment between their corresponding modules.

Definition 54. For a module setting $\chi=\left\langle\theta, \mathcal{D}_{0}, \mathcal{D}_{r}\right\rangle$, let $\operatorname{Ct}(\chi)$ denote the set of constants occurring in $\mathcal{D}_{0}, \mathcal{D}_{r}$, and in the range of $\theta$. A substitution $\mu: \operatorname{Ct}(\chi) \rightarrow \operatorname{Ct}\left(\chi^{\prime}\right)$ is a homomorphism from $\chi$ to $\chi^{\prime}$ if the following conditions hold:

- $\theta \mu=\theta^{\prime}$

- $\mathcal{D}_{0} \mu \subseteq \mathcal{D}_{0}^{\prime}$; and

- $\mathcal{D}_{r} \mu \subseteq \mathcal{D}_{r}^{\prime}$.

We write $\chi \hookrightarrow \chi^{\prime}$ to denote that a homomorphism from $\chi$ to $\chi^{\prime}$ exists.

The fact that $\chi \hookrightarrow \chi^{\prime}$ (witnessed by some homomorphism $\mu$ ) implies that $\chi^{\prime}$ is "more general" than $\chi$, in the sense that any proof in $\mathcal{P}^{\chi} \cup \mathcal{D}_{0}$ of a fact in $\mathcal{D}_{r}$ can be embedded (via $\mu$ ) in a support-preserving way into a proof in $\mathcal{P} \chi^{\prime} \cup \mathcal{D}_{0}^{\prime}$ of a fact in $\mathcal{D}_{r}^{\prime}$. It follows that $\operatorname{supp}(\chi)$ is contained in $\operatorname{supp}\left(\chi^{\prime}\right)($ modulo $\mu)$ and hence we also have $\mathcal{M}^{\chi} \subseteq \mathcal{M}^{\chi^{\prime}}$.

Theorem 55. If $\chi, \chi^{\prime}$ are s.t. $\chi \hookrightarrow \chi^{\prime}$, then $\mathcal{M}^{\chi} \subseteq \mathcal{M}^{\chi^{\prime}}$. 
Proof. Let $\chi=\left\langle\theta, \mathcal{D}_{0}, \mathcal{D}_{r}\right\rangle$ and $\chi^{\prime}=\left\langle\theta^{\prime}, \mathcal{D}_{0}^{\prime}, \mathcal{D}_{r}^{\prime}\right\rangle$, and let $\mu: \operatorname{Ct}(\chi) \rightarrow \operatorname{Ct}\left(\chi^{\prime}\right)$ be a homomorphism from $\chi$ to $\chi^{\prime}$. Since $\mathcal{D}_{r} \mu \subseteq \mathcal{D}_{r}^{\prime}$, it suffices to show that for any rule $r \in \mathcal{O}$ and any proof $\rho$ in $\mathcal{P}^{\chi} \cup \mathcal{D}_{0}$ of a fact $\gamma \in \mathcal{D}_{r}$ such that $\operatorname{supp}(\rho) \cap \Xi^{\chi}(r) \neq \emptyset$, there exists a proof $\rho^{\prime}$ in $\mathcal{P}^{\chi^{\prime}} \cup \mathcal{D}_{0}^{\prime}$ of $\gamma \mu$ such that $\operatorname{supp}\left(\rho^{\prime}\right) \cap \Xi^{\chi^{\prime}}(r) \neq \emptyset$. We prove the following more general claim.

Let $r$ be a rule in $\mathcal{O}$ and $\rho$ a proof of a fact $\gamma$ (not necessarily in $\mathcal{D}_{r}$ ) in $\mathcal{P}^{\chi} \cup \mathcal{D}_{0}$ such that $\operatorname{supp}(\rho) \cap \Xi^{\chi}(r) \neq \emptyset$. We show by induction on the depth $d$ of $\rho$ that there is a proof $\rho^{\prime}$ of $\gamma \mu$ in $\mathcal{P} \chi^{\prime} \cup \mathcal{D}_{0}^{\prime}$ such that $\operatorname{supp}\left(\rho^{\prime}\right) \cap \Xi^{\chi^{\prime}}(r) \neq \emptyset$.

$d=0$

Since, by assumption, $\operatorname{supp}(\rho) \cap \Xi^{\chi}(r) \neq \emptyset$, we have $r=(\rightarrow \psi)$ and $(\rightarrow \gamma) \in \Xi^{\chi}(r)$ with $\gamma=\delta \theta$ for some $\delta \in \psi$, and also $\left(\rightarrow \delta \theta^{\prime}\right) \in \Xi^{\chi^{\prime}}(r)$. Since $\theta$ is defined over all constants in $\mathcal{O}$, it holds that $(\delta \theta) \mu=\delta(\theta \mu)=\delta \theta^{\prime}$, and hence we have a proof of $\gamma \mu$ in $\mathcal{P}^{\chi^{\prime}} \cup \mathcal{D}_{0}^{\prime}$ supported by a rule in $\Xi^{\chi^{\prime}}(r)$.

$d>0$

Let $\rho=(T, \lambda)$ with $v$ the root of $T$ and $w_{1}, \ldots, w_{n}$ the children of $v$. Let $s$ be the rule used to derive $\lambda(v)$ from $\lambda\left(w_{1}\right), \ldots, \lambda\left(w_{n}\right)$. Finally, for each $i$ let $\rho_{i}$ be the subproof of $\lambda\left(w_{i}\right)$. If for any $i$ we have $\operatorname{supp}\left(\rho_{i}\right) \cap \Xi^{\chi}(r) \neq \emptyset$, the claim follows by the induction hypothesis since $s \mu \in \mathcal{P}^{\chi^{\prime}}$. Otherwise, we have $s \in \Xi^{\chi}(r)$. Moreover, by the induction hypothesis, every $\lambda\left(w_{i}\right) \mu$ has a proof in $\mathcal{P}^{\chi^{\prime}} \cup \mathcal{D}_{0}^{\prime}$, and the claim follows since $s \mu \in \Xi^{\chi^{\prime}}(r)$.

It is straightforward to construct homomorphisms between the module settings in Sections 6 and 7 in accordance with the containment relationship of their corresponding inseparability relations. The following result, which establishes the intuitive relationships between our modules, then follows immediately from Theorem 55 .

Corollary 56.

$$
\begin{gathered}
\mathcal{M}^{\chi_{\mathrm{i}}} \subseteq \mathcal{M}^{\chi_{\mathrm{f}}} \subseteq \mathcal{M}^{\chi_{\mathrm{a}}} \subseteq \mathcal{M}^{\chi_{\mathrm{m}}} \subseteq \mathcal{M}^{\chi_{\mathrm{b}}} \\
\mathcal{M}^{\chi_{\mathrm{i}}} \subseteq \mathcal{M}^{\chi_{\mathrm{c}}} \subseteq \mathcal{M}^{\chi_{\mathrm{b}}} \\
\mathcal{M}^{\chi_{\mathrm{wa}}} \subseteq \mathcal{M}^{\chi_{\mathrm{a}}}
\end{gathered}
$$

As already illustrated by examples throughout Sections 6 and 7, these containment relations are strict for many $\mathcal{O}$ and $\Sigma$. Furthermore, it can be easily checked that these containment relations are complete, in the sense that module settings that are unrelated in Corollary 56 (e.g., $\mathcal{M}^{\chi_{\mathrm{f}}}$ and $\mathcal{M}^{\chi_{\mathrm{wq}}}$ ) are incomparable.

\section{Depletingness, Self-Containment, and Justification-Preservation}

The minimal requirement on a module is to preserve all relevant consequences w.r.t. a given inseparability relation. As we argued in Section 3, however, in some applications it is desirable that modules satisfy additional properties. In this section, we establish whether our modules in Sections 6 and 7 satisfy the (strong) depletingness, self-containment, and justification-preservation properties enjoyed by locality-based modules.

To establish our results, it is convenient to abstract away from the notion of module setting for a fixed $\mathcal{O}$ and $\Sigma$ and consider instead families of module settings; that is, functions that assign a module setting to each pair of $\mathcal{O}$ and $\Sigma$. 
Definition 57. A module setting family is a function $\Psi$ that maps each pair of ontology $\mathcal{O}$ and signature $\Sigma$ to a module setting for $\mathcal{O}$ and $\Sigma$. Given an inseparability relation $\mathcal{S}$, we say that $\Psi$ is $\mathcal{S}$-admissible if, for each pair of $\mathcal{O}$ and $\Sigma, \mathcal{M}^{\Psi(\mathcal{O}, \Sigma)}$ is a $\equiv_{\Sigma}^{\mathcal{S}}$-module of $\mathcal{O}$. Furthermore, we say that $\Psi$ is depleting (resp. strongly depleting, self-contained, justification-preserving) if so is $\mathcal{M}^{\Psi(\mathcal{O}, \Sigma)}$ for each $\mathcal{O}$ and $\Sigma$.

Finally, for each $\mathcal{S} \in\{\mathrm{m}, \mathrm{q}, \mathrm{f}, \mathrm{i}, \mathrm{c}, \mathrm{wq}\}$, we denote with $\Psi^{\mathcal{S}}$ the (S-admissible) family induced by the module setting $\chi^{\mathcal{S}}$ as defined in Sections 6 and 7 .

\subsection{Depletingness and Justification-Preservation}

As discussed in Section 3, depletingness of $\mathcal{M}$ ensures that $\mathcal{O} \backslash \mathcal{M}$ is inseparable from the empty ontology and hence no relevant information is left behind in $\mathcal{O}$ after extracting $\mathcal{M}$. As illustrated by the following example, not all modules are depleting.

Example 58. Consider $\mathcal{O}$ consisting of the following rules and let $\Sigma=\{A, B\}$ :

$$
s_{1}=A(x) \rightarrow B(x) \wedge C(x) \quad s_{2}=A(x) \rightarrow D(x) \wedge E(x) \quad s_{3}=D(x) \rightarrow B(x)
$$

Clearly, both $\mathcal{M}_{1}=\left\{s_{1}\right\}$ and $\mathcal{M}_{2}=\left\{s_{2}, s_{3}\right\}$ are implication-inseparable from $\mathcal{O}$ and hence $\equiv_{\Sigma}^{\mathrm{i}}$-modules. However, neither of them is depleting.

We next show that all the modules we defined in Sections 6 and 7 are depleting.

Proposition 59. $\Psi^{\mathcal{S}}$ is depleting for each $\mathcal{S} \in\{\mathrm{m}, \mathrm{q}, \mathrm{f}, \mathrm{i}, \mathrm{c}, \mathrm{wq}\}$.

Proof. Let $\mathcal{O}$ and $\Sigma$ be arbitrary and let $\mathcal{M}=\Psi^{\mathcal{S}}(\mathcal{O}, \Sigma)$. By Theorems 25, 31, 35, 40, 49 and 53, it suffices to show $\Psi^{\mathcal{S}}(\mathcal{O} \backslash \mathcal{M}, \Sigma)=\emptyset$. By the definition of a module (cf. Definition 19), it holds that $\Psi^{\mathcal{S}}(\mathcal{O} \backslash \mathcal{M}, \Sigma) \subseteq \mathcal{O} \backslash \mathcal{M}$. Furthermore, since $\mathcal{O} \backslash \mathcal{M} \subseteq \mathcal{O}$, it follows that $\Psi^{\mathcal{S}}(\mathcal{O} \backslash \mathcal{M}, \Sigma) \subseteq \mathcal{M}$. Consequently, $\Psi^{\mathcal{S}}(\mathcal{O} \backslash \mathcal{M}, \Sigma)=\emptyset$.

We next show that our modules are also justification-preserving and hence can be seamlessly exploited in ontology debugging applications.

Proposition 60. $\Psi^{\mathcal{S}}$ is justification-preserving for each $\mathcal{S} \in\{\mathrm{m}, \mathrm{q}, \mathrm{f}, \mathrm{i}, \mathrm{c}, \mathrm{wq}\}$.

Proof. Let $\phi \in \operatorname{rel}_{\mathcal{S}}(\mathcal{O}, \Sigma)$ and let $\mathcal{O}^{\prime}$ be a justification of $\phi$ in $\mathcal{O}$. We need to check that $\mathcal{O}^{\prime} \subseteq \mathcal{M}^{\Psi^{\mathcal{S}}(\mathcal{O}, \Sigma)}$. Since $\phi \in \operatorname{rel}_{\mathcal{S}}(\mathcal{O}, \Sigma)$ and $\mathcal{O}^{\prime} \models \phi$, it follows by definition of rel $\mathcal{S}_{\mathcal{S}}$ that $\phi \in \operatorname{rel}_{\mathcal{S}}\left(\mathcal{O}^{\prime}, \Sigma\right)$. By Theorems 25, 31, 35, 40, 49 and 53, we have $\mathcal{M}^{\Psi^{\mathcal{S}}\left(\mathcal{O}^{\prime}, \Sigma\right)} \equiv_{\Sigma}^{\mathcal{S}} \mathcal{O}^{\prime}$, and therefore $\mathcal{M}^{\Psi^{\mathcal{S}}\left(\mathcal{O}^{\prime}, \Sigma\right)} \models \phi$. By the definition of a module (cf. Definition 19) it is immediate that $\mathcal{O}^{\prime} \subseteq \mathcal{O}$ implies $\mathcal{M}^{\Psi^{\mathcal{S}}\left(\mathcal{O}^{\prime}, \Sigma\right)} \subseteq \mathcal{M}^{\Psi^{\mathcal{S}}(\mathcal{O}, \Sigma)}$. Finally, by minimality of $\mathcal{O}^{\prime}$, we have $\mathcal{M}^{\Psi^{\mathcal{S}}\left(\mathcal{O}^{\prime}, \Sigma\right)}=\mathcal{O}^{\prime}$ and therefore $\mathcal{O}^{\prime} \subseteq \mathcal{M}^{\Psi^{\mathcal{S}}(\mathcal{O}, \Sigma)}$.

We conclude by addressing the effect of normalisation on these properties. Similarly to our treatment of normalisation in Proposition 13 from Section 3, we show that we can recover a depleting and justification-preserving module for a $\mathcal{S R O I} \mathcal{Q}$ ontology $\mathcal{O}$ from one such module for its normalisation norm $(\mathcal{O})$.

Proposition 61. Let $\mathcal{S}$ be an inseparability relation, and let $\Psi$ be a module setting family that is $\mathcal{S}$-admissible and depleting. Let norm be a normalisation function. Let $\mathcal{O}$ be a $\mathcal{S R O I} \mathcal{Q}$ ontology and let $\mathcal{M} \subseteq \mathcal{O}$ be such that the following holds: 
1. $\mathcal{M}^{\Psi(\operatorname{norm}(\mathcal{O}), \Sigma)} \subseteq \operatorname{norm}(\mathcal{M})$ and

2. $\operatorname{norm}(\mathcal{O} \backslash \mathcal{M}) \subseteq \operatorname{norm}(\mathcal{O}) \backslash \operatorname{norm}(\mathcal{M})$.

Then, $\mathcal{M}$ is a depleting and justification-preserving $\equiv_{\Sigma}^{\mathcal{S}}$-module of $\mathcal{O}$.

Proof. Let $\mathcal{O}^{\prime}=\operatorname{norm}(\mathcal{O})$. Proposition 13 implies that $\mathcal{M}$ is a $\equiv_{\Sigma}^{\mathcal{S}}$-module of $\mathcal{O}^{\prime}$.

We next show that $\mathcal{M}$ is depleting. Since $\mathcal{M}^{\Psi\left(\mathcal{O}^{\prime}, \Sigma\right)} \subseteq \operatorname{norm}(\mathcal{M})$, we have $\mathcal{O}^{\prime} \backslash \operatorname{norm}(\mathcal{M}) \subseteq$ $\mathcal{O}^{\prime} \backslash \mathcal{M}^{\Psi\left(\mathcal{O}^{\prime}, \Sigma\right)}$. Proposition 59 implies that $\mathcal{O}^{\prime} \backslash \mathcal{M}^{\Psi\left(\mathcal{O}^{\prime}, \Sigma\right)} \equiv{ }_{\Sigma}^{\mathcal{S}} \emptyset$, and hence by monotonicity of first-order logic also $\mathcal{O}^{\prime} \backslash \operatorname{norm}(\mathcal{M})=\operatorname{norm}(\mathcal{O} \backslash \mathcal{M}) \equiv{ }_{\Sigma}^{\mathcal{S}} \emptyset$. Since norm $(\mathcal{O} \backslash \mathcal{M})$ is a conservative extension of $\mathcal{O} \backslash \mathcal{M}$, by Definition 4 we have norm $(\mathcal{O} \backslash \mathcal{M}) \equiv_{\Sigma}^{\mathcal{S}} \mathcal{O} \backslash \mathcal{M}$, and thus $\mathcal{O} \backslash \mathcal{M} \equiv_{\Sigma}^{\mathcal{S}} \emptyset$.

To show that $\mathcal{M}$ is justification-preserving, let $\varphi \in \operatorname{rel}_{\mathcal{S}}(\mathcal{O}, \Sigma)$ and consider a justification $\tilde{\mathcal{O}} \subseteq \mathcal{O}$ of $\varphi$ in $\mathcal{O}$. Suppose there is some $\alpha \in \tilde{\mathcal{O}} \backslash \mathcal{M}$. Then $\alpha \in \mathcal{O} \backslash \mathcal{M}$ and $\operatorname{norm}(\alpha) \subseteq \operatorname{norm}(\mathcal{O} \backslash \mathcal{M})=\mathcal{O}^{\prime} \backslash \operatorname{norm}(\mathcal{M})$. Since $\mathcal{M}^{\Psi\left(\mathcal{O}^{\prime}, \Sigma\right)} \subseteq \operatorname{norm}(\mathcal{M})$, this implies norm $(\alpha) \cap \mathcal{M}^{\Psi\left(\mathcal{O}^{\prime}, \Sigma\right)}=\emptyset$. On the other hand, since norm $(\mathcal{O})$ is a conservative extension of $\mathcal{O}$, we have $\varphi \in \operatorname{rel}_{\mathcal{S}}(\operatorname{norm}(\mathcal{O}), \Sigma)$. Also, because norm $(\tilde{\mathcal{O}})$ is a conservative extension of $\tilde{\mathcal{O}}$, we have $\operatorname{norm}(\tilde{\mathcal{O}}) \models \varphi$ and there must be a justification $\tilde{\mathcal{O}}^{\prime}$ of $\varphi$ in $\operatorname{norm}(\tilde{\mathcal{O}}) \subseteq \mathcal{O}^{\prime}$. By Proposition $60, \mathcal{M}^{\Psi\left(\mathcal{O}^{\prime}, \Sigma\right)}$ is justification-preserving, and consequently $\tilde{\mathcal{O}}^{\prime} \subseteq \mathcal{M}^{\Psi\left(\overline{\mathcal{O}}^{\prime}, \Sigma\right)}$. Furthermore, by minimality of $\tilde{\mathcal{O}}$ there is no proper subset of $\tilde{\mathcal{O}}$ whose normalisation includes $\tilde{\mathcal{O}}^{\prime}$. It follows that norm $(\alpha) \cap \tilde{\mathcal{O}}^{\prime} \neq \emptyset$ and hence norm $(\alpha) \cap \mathcal{M}^{\Psi\left(\mathcal{O}^{\prime}, \Sigma\right)} \neq \emptyset$. This is a contradiction that stems from assuming that $\alpha \in \tilde{\mathcal{O}} \backslash \mathcal{M}$. Therefore $\tilde{\mathcal{O}} \subseteq \mathcal{M}$, i.e., $\mathcal{M}$ is justification-preserving.

\subsection{Self-Containment and Strong Depletingness}

In contrast to locality-based modules, the modules obtained using our approach are neither strongly depleting, nor self-contained. To see this, consider the following example.

Example 62. Let $\Sigma=\{A, D\}$ and $\mathcal{O}=\left\{r_{10}-r_{15}\right\}$ with

$$
\begin{aligned}
& r_{10}=(\rightarrow A(o)) \\
& r_{11}=A(x) \rightarrow \exists y \cdot[R(x, y) \wedge B(y)] \\
& r_{12}=A(x) \rightarrow \exists y \cdot[R(x, y) \wedge C(y)] \\
& r_{13}=R(x, y) \rightarrow D(x) \\
& r_{14}=B(x) \rightarrow C(x) \\
& r_{15}=(\rightarrow C(i))
\end{aligned}
$$

Let $\mathcal{M}_{1}=\left\{r_{10}-r_{13}\right\}$ and $\mathcal{M}_{2}=\left\{r_{11}-r_{13}\right\}$. We can check that

$$
\begin{aligned}
& \mathcal{M}^{\chi_{\mathrm{f}}}=\mathcal{M}^{\chi_{\mathrm{q}}}=\mathcal{M}^{\chi_{\mathrm{m}}}=\mathcal{M}^{\chi_{\mathrm{wq}}}=\mathcal{M}_{1} \\
& \mathcal{M}^{\chi_{\mathrm{i}}}=\mathcal{M}^{\chi_{\mathrm{c}}}=\mathcal{M}_{2}
\end{aligned}
$$

Clearly, $\mathcal{O} \models C(i)$ and $\mathcal{M}^{\chi_{\text {wq }}} \not \models C(i)$ where $C$ is in the signature of $\mathcal{M}_{1}$; hence, $\mathcal{M}^{\chi_{\text {wq }}}$ is not self-contained. Furthermore, $\mathcal{M}^{\text {wq }}$ is not strongly depleting since $\mathcal{O} \backslash \mathcal{M}^{\text {wq }}=C(i)$. For the remaining inseparability relations, observe that $\mathcal{O} \models B(x) \rightarrow C(x)$ and $\mathcal{M}_{i} \not \models B(x) \rightarrow C(x)$ for $1 \leq i \leq 2$. Since both $B$ and $C$ are in the signatures of $\mathcal{M}_{1}$ and $\mathcal{M}_{2}$ it follows that none of our modules is self-contained (note that implications are relevant consequences for all relations other than wq). Furthermore, since we also have that $\mathcal{O} \backslash \mathcal{M}_{i} \models B(x) \rightarrow C(x)$, we can conclude that our modules are also not strongly depleting. 
Self-containment and strong depletingness are not always needed for applications. Thus, the fact that our modules do not satisfy them by default can be beneficial as it may allow us to compute smaller modules.

However, as mentioned in Section 3, these properties can be useful in certain ontology reuse scenarios. We next show that our framework can be adapted so as to satisfy these properties whenever they are required. This can be achieved via a fixpoint construction where modules are computed w.r.t. iterative extensions of the initial signature. Such fixpoint constructions are reminiscent of the standard algorithms for computing locality modules (Cuenca Grau et al., 2007a, 2008).

Definition 63. Let $\Psi^{\mathcal{S}}$ be a module setting family for an inseparability relation $\mathcal{S}$. We define the family $\Psi_{\text {self }}^{\mathcal{S}}$ as the function mapping each $\mathcal{O}$ and $\Sigma$ to the least fixpoint of the sequence $\left\{\mathcal{M}_{i}\right\}_{i \geq 0}$ as defined next:

$$
\begin{aligned}
& \Sigma_{0}=\Sigma \\
& \Sigma_{i}=\Sigma_{i-1} \cup \operatorname{Sig}\left(\mathcal{M}_{i-1}\right) \text { for } i>0 \quad \mathcal{M}_{i}=\mathcal{M}^{\Psi^{\mathcal{S}}\left(\mathcal{O}, \Sigma_{i}\right)} \text { for } i \geq 0
\end{aligned}
$$

The aforementioned fixpoint is well-defined: since $\Sigma_{i} \subseteq \Sigma \cup \operatorname{Sig}(\mathcal{O})$ for each $i \geq 0$ and $\Sigma \cup \operatorname{Sig}(\mathcal{O})$ is finite, there must be some $i_{0} \geq 0$ such that $\Sigma_{i_{0}}=\Sigma_{j}$ and $\mathcal{M}_{i_{0}}=\mathcal{M}_{j}$ for each $j>i_{0}$. We show that, with this adaptation, our modules satisfy the required properties.

Proposition 64. $\Psi_{\text {self }}^{\mathcal{S}}$ is self-contained and strongly depleting for each $\mathcal{S} \in\{\mathrm{m}, \mathrm{q}, \mathrm{f}, \mathrm{i}, \mathrm{c}, \mathrm{wq}\}$.

Proof. Let $\mathcal{M}=\Psi_{\text {self }}^{\mathcal{S}}(\mathcal{O}, \Sigma)$. It is immediate that $\mathcal{M}$ is a self-contained $\equiv_{\Sigma}^{\mathcal{S}}$-module of $\mathcal{O}$. Strong depletingness of $\mathcal{M}$ follows from Proposition 59 for $\mathcal{S} \in\{\mathrm{m}, \mathrm{q}, \mathrm{f}, \mathrm{i}, \mathrm{c}, \mathrm{wq}\}$.

The construction in Definition 63 can straightforwardly be adapted to the case of nonnormalised $\mathcal{S} \mathcal{R O I} \mathcal{Q}$ ontologies by following the same approach as in Proposition 61.

\section{Complexity of Module Extraction}

In this section, we argue that our modules can be efficiently computed in many practically relevant cases. For this, we analyse the complexity of the following decision problem.

Definition 65. Let $L$ be a class of ontologies and let $\mathcal{S} \in\{\mathrm{m}, \mathrm{q}, \mathrm{f}, \mathrm{i}, \mathrm{c}, \mathrm{b}, \mathrm{wq}\}$ be an inseparability relation. The decision problem isInModule $[L, \mathcal{S}]$ is as follows:

- Input: an ontology $\mathcal{O} \in L$, a signature $\Sigma$ and a rule $r \in \mathcal{O}$.

- Output: TRUE if and only if $r \in \mathcal{M}^{\Psi^{\mathcal{S}}(\mathcal{O}, \Sigma)}$.

Furthermore, we consider the following classes of ontologies, which are strongly connected to DL-based ontology languages.

Definition 66. Let $k$ be a fixed non-negative integer. The class $L_{\text {arity }}^{k}$ consists of all ontologies where predicates have arity at most $k$.

The graph of a conjunction of atoms $\varphi$ is the undirected graph $G_{\varphi}=(V, E)$ such that $V$ is the set of variables occurring in $\varphi$, and $E$ contains an edge between each pair of variables 
that occur together in some atom in $\varphi$. A tree decomposition of $G_{\varphi}$ is a tree $T=(W, F)$, such that there exists a labelling $\lambda$ mapping each vertex $w \in W$ to some subset $\lambda(w) \subseteq V$, and the following conditions are satisfied:

- for each $v \in V$, there exists $v \in W$ with $v \in \lambda(w)$,

- for each $\left\{v, v^{\prime}\right\} \in E$, there exists $w \in W$ with $\left\{v, v^{\prime}\right\} \subseteq \lambda(w)$, and

- for each $v \in V$, the set $\{w \in W \mid v \in \lambda(w)\}$ induces a (connected) subtree of $T$.

The width of the tree decomposition $T$ is $\max _{w \in W}(|\lambda(w)|-1)$. The treewidth of $\varphi$ is the minimum width over all the tree decompositions of $G_{\varphi}$. The treewidth of a rule is defined as the treewidth of its body. Finally, the class $L_{t w}^{k}$ consists of all ontologies where each rule has treewidth at most $k$.

The rules correspong to $\mathcal{S R O I \mathcal { Q }}$ ontologies are not only of fixed predicate arity, but also their bodies are tree-shaped (see Section 2.2). The latter implies that rules stemming from $\mathcal{S R O I} \mathcal{Q}$ ontologies have treewidth at most one.

As already discussed, an appealing feature of our approach is that module extraction can be delegated to an off-the-shelf datalog reasoner, regardless of the language in which ontologies are expressed. The following proposition establishes that both the datalog program and the initial dataset exploited in our approach are of polynomial size; furthermore, the datalog transformation $\Xi^{\chi}$ in the definition of a module setting (see Definition 19 in Section 5) does not alter the shape of rules in the original ontology in any significant way.

Proposition 67. Let $\mathcal{O}$ be an ontology and $\Sigma \subseteq \operatorname{Sig}(\mathcal{O})$ a signature. Furthermore, let $\mathcal{S} \in\{\mathrm{m}, \mathrm{q}, \mathrm{f}, \mathrm{i}, \mathrm{c}, \mathrm{b}, \mathrm{wq}\}$ and $\Psi_{\mathcal{S}}(\mathcal{O}, \Sigma)=\chi=\left\langle\theta, \mathcal{D}_{0}, \mathcal{D}_{r}\right\rangle$. Then, $\mathcal{P}^{\chi}$ and $\mathcal{D}_{0}$ are of size linear in $|\mathcal{O}|$. Furthermore, if $\mathcal{O}$ is in $L_{\text {arity }}^{k}$ (resp. in $L_{t w}^{k}$ ) for some fixed $k$, then so is $\mathcal{P}^{\chi}$.

Proof. It is clear from Definition 5 that $\Xi^{\chi}(r)$ contains a datalog rule for each atom in the head of $r$. Thus, $\mathcal{P} \chi$ is clearly of size linear w.r.t. $|\mathcal{O}|$. Furthermore, $\mathcal{D}_{0}$ contains one fact for each predicate in $\Sigma$, and since $\Sigma \subseteq \operatorname{Sig}(\mathcal{O})$, it follows that $\mathcal{D}_{0}$ is also of size linear w.r.t. $|\mathcal{O}|$. Finally, the transformation $\Xi^{\chi}$ does not increase the arity of predicates and the body of each rule $\Xi^{\chi}(r)$ coincides with that of $r$ and hence preserves its treewidth.

The computational properties of datalog programs of bounded arity and/or treewidth are well-understood: fact entailment is NP-complete in combined complexity for programs of bounded arity, and the complexity drops to PTIME if we additionally restrict ourselves to programs of bounded treewidth. Bounded arity of predicates implies that the corresponding materialisation is polynomially bounded in size, and thus can be computed in a polynomial number of steps (i.e., applications of the immediate consequence operator); furthermore, bounded treewidth of rule bodies implies that each such step can be performed in polynomial time (Grohe, Schwentick, \& Segoufin, 2001; Chekuri \& Rajaraman, 2000).

The complexity of module extraction, however, is not only determined by that of datalog reasoning, but also by the complexity of computing the support of all proofs involved in a relevant entailment.

The following theorem, proved by Zhou et al. (2014), establishes that computing such support can also be reduced to standard datalog reasoning. Given a program $\mathcal{P}$, a dataset 
$\mathcal{D}$, and a set of facts $F$, the main idea is to extend $\mathcal{P}$ and $\mathcal{D}$ with additional rules and facts that are responsible for computing the support of all proofs of facts from $F$ in $\mathcal{P} \cup \mathcal{D}$. Such support is "recorded" by means of fresh predicates: auxiliary predicates $\bar{Q}$ are used to record relevant facts in $\mathcal{D}$ of the form $Q(\mathbf{c})$; furthermore, each rule $r \in \mathcal{P}$ is represented by a fresh constant $d_{r}$, and a fresh unary predicate Rel is used to capture the relevant rules from $\mathcal{P}$ in the support.

Theorem 68 (Zhou et al., 2014). Let $\mathcal{P}$ be a datalog program, let $\mathcal{D}$ be a dataset, and let $F$ be a set of facts in the materialisation of $\mathcal{P} \cup \mathcal{D}$. Let Rel be a fresh unary predicate and, for each predicate $Q$ occurring in $\mathcal{P} \cup \mathcal{D}$, let $\bar{Q}$ be a fresh predicate of the same arity. Furthermore, let $d_{r}$ be a fresh constant for each $r \in \mathcal{P}$.

Let $\Delta(\mathcal{D}, F)$ be the dataset $\Delta(\mathcal{D}, F)=\mathcal{D} \cup\{\bar{P}(\mathbf{c}) \mid P(\mathbf{c}) \in F\}$ and let $\Delta(\mathcal{P})$ be the smallest datalog program including $\mathcal{P}$ and containing all of the following rules for each $r=\bigwedge_{j=1}^{m} B_{1}\left(\mathbf{x}_{j}\right) \rightarrow H(\mathbf{x})$ in $\mathcal{P}:$

$$
\begin{aligned}
& \bar{H}(\mathbf{x}) \wedge B_{1}\left(\mathbf{x}_{1}\right) \wedge \ldots \wedge B_{m}\left(\mathbf{x}_{m}\right) \rightarrow \operatorname{Rel}\left(d_{r}\right) \\
& \bar{H}(\mathbf{x}) \wedge B_{1}\left(\mathbf{x}_{1}\right) \wedge \ldots \wedge B_{m}\left(\mathbf{x}_{m}\right) \rightarrow \bar{B}_{j}\left(\mathbf{x}_{1}\right) \\
& \bar{H}(\mathbf{x}) \wedge B_{1}\left(\mathbf{x}_{1}\right) \wedge \ldots \wedge B_{m}\left(\mathbf{x}_{m}\right) \rightarrow \bar{B}_{j}\left(\mathbf{x}_{m}\right)
\end{aligned}
$$

Then, a rule $s \in \mathcal{P}$ is in the support of some proof in $\mathcal{P} \cup \mathcal{D}$ of a fact from $F$ iff $\operatorname{Rel}\left(d_{s}\right)$ is in the materialisation of $\Delta(\mathcal{P}) \cup \Delta(\mathcal{D}, F)$.

The datalog program $\Delta(\mathcal{P})$ (resp. the dataset $\Delta(\mathcal{D}, F)$ ) in Theorem 68 is of size polynomial in $|\mathcal{P}|$ (resp. in $|\mathcal{D}|$ and $|F|$ ) and does not use any predicates with arity greater than that of predicates used in $\mathcal{P}$. However, $\Delta(\mathcal{P})$ may have a larger treewidth than $\mathcal{P}$. We next argue that in the case of $\mathcal{S R O I} \mathcal{Q}$ ontologies the increase in treewidth is bounded.

Proposition 69. Let $\mathcal{O}$ be a normalised $\mathcal{S R O} \mathcal{I} \mathcal{Q}$ ontology and $\Sigma \subseteq \operatorname{Sig}(\mathcal{O})$ a signature. Furthermore, let $\chi$ be a module setting for $\mathcal{O}$ and $\Sigma$. Then $\Delta\left(\mathcal{P}^{\chi}\right)$ has treewidth at most 2 .

Proof. For each rule $r \in \mathcal{O}$ whose head is formed only by (one or more) atoms that are unary, or mention no more than one variable, it is straightforward that $\Delta\left(\Xi^{\chi}(r)\right)$ still consists only of rules with tree-shaped bodies. For each $r \in \mathcal{O}$ that does not mention more than two variables, it is also straightforward that $\Delta\left(\Xi^{\chi}(r)\right)$ also consists only of rules with tree-shaped bodies. Finally, if $r$ mentions more than two variables, and its head contains atoms that mention more than one variable, then $r$ must be of one of the following forms:

- $A(x) \wedge \bigwedge_{i=1}^{m+1}\left[R\left(x, y_{i}\right) \wedge B\left(y_{i}\right)\right] \rightarrow \bigvee_{i \neq j} y_{i} \approx y_{j}$

Then, the bodies of the rules in $\Delta\left(\Xi^{\chi}(r)\right)$ are of the form

$$
A(x) \wedge \bigwedge_{i=1}^{m+1}\left[R\left(x, y_{i}\right) \wedge B\left(y_{i}\right)\right] \wedge y_{i_{1}} \approx y_{i_{2}} \quad 1 \leq i_{1}<i_{2} \leq m
$$

Hence, they have treewidth 2 due to the cycle of length 3 formed by $R\left(x, y_{i_{1}}\right), R\left(x, y_{i_{2}}\right)$ and $y_{i_{1}} \approx y_{i_{2}}$. 
- $R_{1}(x, y) \wedge R_{2}(y, z) \rightarrow S(x, z)$

The body of the single rule in $\Delta\left(\Xi^{\chi}(r)\right)$ will be of the form $R_{1}(x, y) \wedge R_{2}(y, z) \wedge S(x, z)$, which has treewidth 2 due to the cycle of length 3 formed by its three atoms.

- The cases involving equality in the body of rules, namely $x \approx y \wedge y \approx z \rightarrow x \approx z$, $A\left(x_{1}, x_{2}\right) \wedge x_{1} \approx y \rightarrow A\left(y, x_{2}\right)$, and $A\left(x_{1}, x_{2}\right) \wedge x_{2} \approx y \rightarrow A\left(x_{1}, y\right)$, are analogous to the previous case.

The following theorem establishes two practically relevant cases for which module extraction in our framework can be performed in polynomial time. We first show that modules $\mathcal{M}^{\chi_{\mathrm{m}}}$ ensuring model-inseparability are computable in polynomial time for arbitrary ontologies and signatures. Then, we establish that modules $\mathcal{M}^{\chi}$ for the remaining inseparability relations considered in this paper are also computable in polynomial time for all classes of ontologies whose extended datalog program $\Delta\left(\mathcal{P}^{\chi}\right)$ in Definition 68 can be bounded in both predicate arity and treewidth.

Theorem 70. Let $L$ be a class of ontologies, $\mathcal{S} \in\{\mathrm{m}, \mathrm{q}, \mathrm{f}, \mathrm{i}, \mathrm{c}, \mathrm{wq}\}$, and $L_{\mathcal{S}, \Delta}$ the following class of datalog programs:

$$
L_{\mathcal{S}, \Delta}=\left\{\Delta\left(\mathcal{P}^{\Psi^{\mathcal{S}}(\mathcal{O}, \Sigma)}\right) \mid \mathcal{O} \in L, \Sigma \subseteq \operatorname{Sig}(\mathcal{O})\right\}
$$

The problem islnModule $[L, \mathcal{S}]$ is decidable in polynomial time if either of the following conditions is satisfied:

- $\mathcal{S}=\mathrm{m}$, or

- $L_{\mathcal{S}, \Delta} \subseteq L_{\text {arity }}^{k} \cap L_{t w}^{k^{\prime}}$ for some fixed non-negative integers $k$ and $k^{\prime}$.

Proof. Consider an arbitrary ontology $\mathcal{O}$ and a signature $\Sigma$. Let $\Psi^{\mathcal{S}}(\mathcal{O}, \Sigma)=\left\langle\theta, \mathcal{D}_{0}, \mathcal{D}_{r}\right\rangle$ $\mathcal{P}=\mathcal{P}^{\Psi^{\mathcal{S}}(\mathcal{O}, \Sigma)}$, and $F=\mathcal{D}_{r} \cap \mathcal{P}\left(\mathcal{D}_{0}\right)$. By Proposition $67, \mathcal{P}$ and $\mathcal{D}_{0}$ can be computed in time polynomial in the size of $\mathcal{O}$, and so can $\Delta(\mathcal{P})$. Therefore, it suffices to show that $\Delta\left(\mathcal{D}_{0}, F\right)$ can be obtained in polynomial time and so can the materialisation of $\Delta(\mathcal{P}) \cup \Delta\left(\mathcal{D}_{0}, F\right)$.

If $\mathcal{S}=\mathrm{m}$ then $\mathcal{D}_{r}$ and $\Delta\left(\mathcal{D}_{0}, F\right)$ can be computed in linear time. Furthermore, it is easy to see that computing the materialisation of $\Delta(\mathcal{P}) \cup \Delta\left(\mathcal{D}_{0}, F\right)$ is also feasible in linear time since it boils down to propositional datalog reasoning.

Consider now $\mathcal{S} \in\{\mathrm{q}, \mathrm{f}, \mathrm{i}, \mathrm{c}, \mathrm{wq}\}$. Note that $\Delta(\mathcal{P}) \in L_{\text {arity }}^{k} \cap L_{\text {tw }}^{k^{\prime}}$ implies $\mathcal{P} \in L_{\text {arity }}^{k} \cap L_{t w}^{k^{\prime}}$. Hence, $\mathcal{P}\left(\mathcal{D}_{0}\right)$ can be computed in time polynomial in the size of $\mathcal{O}$. Since $F$ is always a set of facts in $\mathcal{P}\left(\mathcal{D}_{0}\right)$ over predicates from $\Sigma$, it can be computed in polynomial time. Moreover, the dataset $\Delta\left(\mathcal{D}_{0}, F\right)$ can be computed in polynomial time as well and thus, since $\Delta(\mathcal{P}) \in L_{\text {arity }}^{k} \cap L_{t w}^{k^{\prime}}$, so can the materialisation of $\Delta(\mathcal{P}) \cup \Delta\left(\mathcal{D}_{0}, F\right)$.

Tractability of module extraction w.r.t. $\mathcal{S R O} \mathcal{I} \mathcal{Q}$ ontologies is now an immediate consequence of Theorem 70 and Proposition 69.

Corollary 71. Let $\mathcal{S} \in\{\mathrm{m}, \mathrm{q}, \mathrm{f}, \mathrm{i}, \mathrm{c}, \mathrm{wq}\}$ and let $\mathcal{O}$ be a normalised $\mathcal{S R O} \mathcal{I} \mathcal{Q}$ ontology. The module $\mathcal{M}^{\chi \mathcal{S}}$ is computable in polynomial time. 


\section{Optimality}

As already discussed, in general, our modules are not minimal for their corresponding inseparability relation. It is, however, of interest to determine which module setting families yield the smallest possible modules for a given inseparability relation within the limits of our framework. To this end, we next present and study a suitable notion of optimality applicable to module setting families.

Our notion of module setting family in Definition 57 is rather general in that it does not establish any relationship between the different module settings in the family. In order to study optimality, it makes sense to restrict ourselves to families satisfying certain uniformity conditions. Roughly speaking, we consider a family as uniform if (i) existentially quantified variables and constants in ontologies are treated homogeneously within a setting (i.e., different existential variables receive the same treatment, and so do different constants) as well as consistently across different settings; and (ii) signatures are treated monotonically across settings (i.e., if $\chi$ and $\chi^{\prime}$ are members of a family for some ontology $\mathcal{O}$ and signatures $\Sigma$ and $\Sigma^{\prime}$ with $\Sigma \subseteq \Sigma^{\prime}$, then they treat predicates in $\Sigma$ and $\operatorname{Sig}(\mathcal{O}) \backslash \Sigma^{\prime}$ in exactly the same way).

Definition 72. A module setting family $\Psi$ is uniform if, for each pair of ontologies $\mathcal{O}, \mathcal{O}^{\prime}$ and signatures $\Sigma, \Sigma^{\prime}$, the module settings $\Psi(\mathcal{O}, \Sigma)=\left\langle\theta, \mathcal{D}_{0}, \mathcal{D}_{r}\right\rangle$ and $\Psi\left(\mathcal{O}^{\prime}, \Sigma^{\prime}\right)=\left\langle\theta^{\prime}, \mathcal{D}_{0}^{\prime}, \mathcal{D}_{r}^{\prime}\right\rangle$ satisfy the following properties, where $\operatorname{Ex}(\mathcal{F})$ denotes the set of existentially quantified variables in $\mathcal{F}$ :

1. If $\Sigma=\Sigma^{\prime},|\operatorname{Ct}(\mathcal{O})| \leq\left|\operatorname{Ct}\left(\mathcal{O}^{\prime}\right)\right|$ and $|\operatorname{Ex}(\mathcal{O})| \leq\left|\operatorname{Ex}\left(\mathcal{O}^{\prime}\right)\right|$, then there exists an injective substitution $\nu: \operatorname{dom}(\theta) \rightarrow \operatorname{dom}\left(\theta^{\prime}\right)$ mapping variables to variables and constants to constants such that

- $\theta=\nu \theta^{\prime}$,

- $\mathcal{D}_{0}=\left\{A(\mathbf{c}) \mid A(\mathbf{c} \nu) \in \mathcal{D}_{0}^{\prime}\right.$, c has only constants from $\left.\operatorname{Ct}(\Psi(\mathcal{O}, \Sigma))\right\}$, and

- $\mathcal{D}_{r}=\left\{A(\mathbf{c}) \mid A(\mathbf{c} \nu) \in \mathcal{D}_{r}^{\prime}\right.$, c has only constants from $\left.\operatorname{Ct}(\Psi(\mathcal{O}, \Sigma))\right\}$.

2. If $\mathcal{O}=\mathcal{O}^{\prime}$ and $\Sigma \subseteq \Sigma^{\prime}$, then

- $\theta=\theta^{\prime}$

- $\mathcal{D}_{0}=\left\{A(\mathbf{c}) \in \mathcal{D}_{0}^{\prime} \mid A \in \Sigma\right\}$,

- $\left\{A(\mathbf{c}) \in \mathcal{D}_{r} \mid A \in \Sigma\right\}=\left\{A(\mathbf{c}) \in \mathcal{D}_{r}^{\prime} \mid A \in \Sigma\right\}$, and

- $\left\{A(\mathbf{c}) \in \mathcal{D}_{r} \mid A \in \operatorname{Sig}(\mathcal{O}) \backslash \Sigma^{\prime}\right\}=\left\{A(\mathbf{c}) \in \mathcal{D}_{r}^{\prime} \mid A \in \operatorname{Sig}(\mathcal{O}) \backslash \Sigma^{\prime}\right\}$.

For $\mathcal{S}$ an inseparability relation, let $\boldsymbol{\Psi}_{\mathcal{S}}$ be the class of all uniform module setting families that are $\mathcal{S}$-admissible. We say that $\Psi$ is $\mathcal{S}$-optimal if $\Psi \in \Psi_{\mathcal{S}}$ and $\mathcal{M}^{\Psi(\mathcal{O}, \Sigma)} \subseteq \mathcal{M}^{\Psi^{\prime}(\mathcal{O}, \Sigma)}$ for every $\Psi^{\prime} \in \Psi_{\mathcal{S}}$ and each pair of $\mathcal{O}$ and $\Sigma$.

It is easy to see that each of the $\mathcal{S}$-admissible families $\Psi^{\mathcal{S}}$, with $\mathcal{S} \in\{\mathrm{m}, \mathrm{q}, \mathrm{f}, \mathrm{i}, \mathrm{c}, \mathrm{wq}\}$, is uniform. Furthermore, the following theorem establishes that $\Psi^{\mathrm{m}}, \Psi^{\mathrm{i}}, \Psi^{\mathrm{c}}$, and $\Psi^{\mathrm{wq}}$ are also optimal for their respective inseparability relations. The proof of the theorem is rather technical and is deferred to the appendix. 
Theorem 73. The family $\Psi^{\mathcal{S}}$ is $\mathcal{S}$-optimal for $\mathcal{S} \in\{\mathrm{m}, \mathrm{i}, \mathrm{c}, \mathrm{wq}\}$.

In contrast, the families $\Psi^{\mathrm{f}}$ and $\Psi^{\mathrm{q}}$ for fact and query inseparability are not optimal. To see this, consider the ontology $\mathcal{O}$ consisting of the following rules:

$$
A(x) \rightarrow B(x) \quad B(x) \rightarrow A(x) \quad C(x) \rightarrow D(x)
$$

Furthermore, let $\Sigma=\{A, C, D\}$. The module setting $\chi_{\mathrm{f}}=\Psi^{\mathrm{f}}(\mathcal{O}, \Sigma)$ yields $\mathcal{M}^{\chi_{\mathrm{f}}}=\mathcal{O}$. Indeed, for $\mathcal{D}=\{A(a)\}$ we have a non-trivial proof of $A(a)$ in $\mathcal{O} \cup \mathcal{D}$ that involves rules $A(x) \rightarrow B(x)$ and $B(x) \rightarrow A(x)$, which are then included in the module. However, it is clear that $\mathcal{M}=\{C(x) \rightarrow D(x)\}$ is already $\Sigma$-fact inseparable from $\mathcal{O}$.

We now define a different setting $\chi=\left\langle\theta, \mathcal{D}_{0}, \mathcal{D}_{r}\right\rangle$, whose corresponding module is precisely $\mathcal{M}$. For this, the idea is to define $\mathcal{D}_{0}$ and $\mathcal{D}_{r}$ in such a way that the aforementioned proofs of tautological statements are avoided. Consider $\mathcal{D}_{0}$ and $\mathcal{D}_{r}$ as follows:

$$
\begin{aligned}
& \mathcal{D}_{0}=\left\{X\left(c_{Y}^{0}\right) \mid X, Y \in \Sigma\right\} \cup\left\{X\left(c_{Y}^{1}\right) \mid X, Y \in \Sigma \text { and } X \neq Y\right\} \\
& \mathcal{D}_{r}=\left\{Y\left(c_{Y}^{1}\right) \mid Y \in \Sigma\right\}
\end{aligned}
$$

Datasets $\mathcal{D}_{0}$ and $\mathcal{D}_{r}$ are disjoint, which guarantees that proofs of $\Sigma$-tautologies are not taken into account and therefore $\mathcal{M}^{\chi}$ is indeed $\mathcal{M}$. The construction of $\mathcal{D}_{0}$ and $\mathcal{D}_{r}$ given for this example ontology and signature can be generalised so as to define a uniform module setting family $\Psi$ that provides a counter-example to the optimality of $\Psi^{f}$. There is, however, a price to pay for such smaller modules, namely an increase in the size of module settings. Indeed, $\Psi(\mathcal{O}, \Sigma)$ is of size exponential in $\Sigma$, whereas the size of $\Psi^{\mathrm{f}}(\mathcal{O}, \Sigma)$ remains polynomial. Such exponential blowup is clearly undesirable in practice.

The following theorem establishes that $\Psi^{\mathrm{f}}$ and $\Psi^{\mathrm{q}}$ are not optimal. They do, however, work well in practice, as shown in the evaluation presented in Section 13. The proof of the theorem works by proposing "better" module setting families which, in both cases, incur the aforementioned exponential blowup. We conjecture that such a blowup is unavoidable in any optimal module setting family for fact or query inseparability (if such a family exists). As in the case of Theorem 73, the proof is technical and is deferred to the appendix.

Theorem 74. The family $\Psi^{\mathcal{S}}$ is not $\mathcal{S}$-optimal for $\mathcal{S} \in\{\mathrm{f}, \mathrm{q}\}$.

\section{Related Work}

Module extraction has received a great deal of attention in the literature. In Section 12.1 we discuss the complexity of inseparability checking for different ontology languages and inseparability relations. In Section 12.2 we recapitulate existing module extraction techniques based on inseparability, and provide a brief overview of their practical applications. Finally, in Section 12.3 we discuss a number of related problems, such as forgetting, uniform interpolation, and partition-based reasoning.

\subsection{Inseparability Relations}

Inseparability relations originate in the notions of model and deductive conservative extensions for description and modal logics (Antoniou \& Kehagias, 2000; Ghilardi, Lutz, \& 
Wolter, 2006a; Ghilardi, Lutz, Wolter, \& Zakharyaschev, 2006b), and constitute the foundation of module extraction techniques (Konev et al., 2009).

Model inseparability is undecidable for all DLs that extend $\mathcal{E} \mathcal{L}$ (Lutz \& Wolter, 2010). It is, however, tractable for $\mathcal{E} \mathcal{L} \mathcal{I}$ ontologies that are acyclic (Konev et al., 2013); furthermore, it is CONEXPTImE ${ }^{\mathrm{NP}}$-complete for the description logic $\mathcal{A L C I}$ if signatures are restricted to consist of atomic concepts only (Konev et al., 2013). For DL-Lite $\mathcal{b o o l}_{\text {Nol }}^{\mathcal{N}}$ and DL-Lite $e_{\text {horn }}^{\mathcal{N}}$ it is CONEXPTime-hard (Kontchakov et al., 2010), but no matching upper bound is known to the best of our knowledge.

The complexity of query inseparability has been studied mainly for lightweight description logics. It is ExPTImE-complete for $\mathcal{E} \mathcal{L}$ (Lutz \& Wolter, 2010), $\Pi_{2}^{p}$-complete and CONP-complete for $D L$-Lite $e_{\text {bool }}^{\mathcal{N}}$ and $D L$-Lite $e_{\text {horn }}^{\mathcal{N}}$, respectively (Kontchakov et al., 2010),

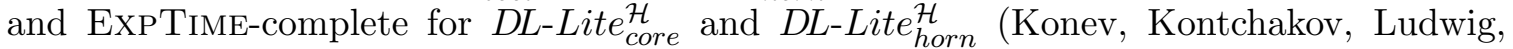
Schneider, Wolter, \& Zakharyaschev, 2011; Botoeva et al., 2014). Baader et al. (2010) considered a variant of query inseparability where the signature of datasets is restricted to $\Sigma$ but the signature of queries is not, and identified decidable sufficient conditions for such inseparability in $\mathcal{E} \mathcal{L} \mathcal{I}$, which can be checked in polynomial time for $\mathcal{E} \mathcal{L}$. The complexity of weak query inseparability (see Section 7.2) was studied by Botoeva et al. (2014); it is known to be P-complete for DL-Lite core,$D L$-Lite $_{\text {horn }}$ and $\mathcal{E} \mathcal{L} \mathcal{H}$, ExPTIME-complete for $D L-L i t e_{\text {core }}^{\mathcal{H}}$ and $D L-L i t e_{\text {horn }}^{\mathcal{H}}$, and 2ExPTIME-complete for Horn- $\mathcal{A} \mathcal{L C H} \mathcal{I}$ and Horn- $\mathcal{A L C \mathcal { I }}$.

The complexity of implication and classification inseparability coincides with that of standard reasoning tasks such as subsumption checking (Konev et al., 2009). In the description logic literature implication inseparability has been studied in a more general form: given $\mathcal{L}$-ontologies $\mathcal{O}$ and $\mathcal{O}^{\prime}$ and a signature $\Sigma$, the problem is to determine whether $\mathcal{O}$ and $\mathcal{O}^{\prime}$ entail the same concept inclusion axioms $C \sqsubseteq D$ where $C$ and $D$ are (possibly complex) $\mathcal{L}$-concepts over $\Sigma$. In this setting, inseparability has been found to be $\Pi_{2}^{p}$-complete for $D L$-Lite bool $^{\mathcal{N}}$, CONP-complete for DL-Lite horn $_{\text {Non }}^{\mathcal{N}}$ (Kontchakov et al., 2010), ExPTIME-complete for $\mathcal{E} \mathcal{L}$ (Lutz \& Wolter, 2010), 2ExpTime-complete for $\mathcal{A L C}$ (Ghilardi et al., 2006a), $\mathcal{A} \mathcal{L C} \mathcal{I}$, $\mathcal{A L C} \mathcal{Q}$ and $\mathcal{A L C} \mathcal{L I}$ (Konev et al., 2009), and undecidable for $\mathcal{A L C} \mathcal{Q} \mathcal{I O}$ (Konev et al., 2009). Note that this variant of implication inseparability is highly dependent on the ontology language, whereas our results are largely logic-independent. We leave the investigation of such inseparability relations within our framework as an interesting problem for future work.

\subsection{Module Extraction}

Practical module extraction techniques are typically based on approximations, which ensure that the computed module is (model) inseparable from the given ontology, yet not necessarily minimal. One such approximation, which we discussed in detail in Section 3, is based on syntactic locality (Cuenca Grau et al., 2007a, 2008; Sattler et al., 2009). An implementation of $\perp-, T$ - and $\perp T^{*}$-module extraction is integrated in the OWL API (Horridge \& Bechhofer, 2011), and an alternative implementation can be downloaded as a separate Java library. ${ }^{3}$ The semantic counterpart of syntactic locality, semantic locality, was proposed in the work of Cuenca Grau et al. (2007b). Deciding semantic locality is, for any given DL, as hard as checking satisfiability w.r.t. the empty TBox, and hence only tractable for logics with restricted expressivity. For this reason modules based on syntactic locality, which

3. https://www.cs.ox.ac.uk/isg/tools/ModuleExtractor/ 
can be extracted in polynomial time, have been the preferred choice in practice. Furthermore, an exhaustive comparison of syntactic and semantic locality modules revealed that the difference between them is not significant in most practical cases (Del Vescovo et al., 2013).

Reachability-based modules (Suntisrivaraporn, 2008; Nortje, Britz, \& Meyer, 2012; Nortje et al., 2013) are a refinement of syntactic locality modules. Available in the same three flavours $\left(\perp-, T\right.$ - and $\perp T^{*}$-reachability), they can also be computed in polynomial time. While $\perp$-reachability modules coincide with $\perp$-modules, $T$ - and $\perp T^{*}$-reachability modules are generally subsets of their syntactic locality counterparts. This refinement comes at the cost of losing depletingness, although reachability modules are still self-contained and preserve all justifications for consequences over the reference signature $\Sigma$.

Konev et al. (2013) and Gatens et al. (2014) developed module extraction techniques for acyclic $\mathcal{E} \mathcal{L} \mathcal{I}$ and acyclic $\mathcal{A L C} \mathcal{Q I}$, respectively. These techniques ensure that modules are self-contained and depleting, and in the case of $\mathcal{E} \mathcal{L I}$ also minimal. The polynomial algorithm for $\mathcal{E} \mathcal{L I}$ is implemented in the system MEX, the more general, non-tractable algorithm for $\mathcal{A L C Q \mathcal { I }}$ is implemented in the system AMEX. In contrast to locality and reachability modules, the applicability of these techniques is limited to a relatively restricted class of ontologies, and tractability is only guaranteed for an even more restricted class.

Kontchakov et al. (2010) exploited the decidability of query inseparability for $D L-L i t e_{\text {bool }}^{\mathcal{N}}$ and $D L-L i t e_{\text {horn }}^{\mathcal{N}}$ for module extraction. Their techniques yield minimal or minimal depleting modules and have the same complexity as the corresponding inseparability relation ( $\Pi_{2}^{p}$-complete and coNP-complete, respectively).

Baader et al. (2010) proposed exponential-time algorithms for extracting modules from $\mathcal{E} \mathcal{L} \mathcal{I}$ ontologies that preserve a variant of query inseparability. Furthermore, they showed that computing such modules is feasible in polynomial time for $\mathcal{E} \mathcal{L}$ ontologies.

Recently, Rousset and Ulliana (2015) studied modularity in the context of deductive triple stores, that is, RDF triple stores equipped with a set of datalog rules. The preservation properties of such modules, however, are very different from the ones considered in our work.

Del Vescovo et al. (2011) considered the problem of finding a polynomial representation of all modules of an ontology, for a particular notion of module. The proposed representation is called atomic decomposition and is applicable to any notion of a module that satisfies certain properties that include self-containment and depletingness. The atomic decomposition of an ontology for a suitable notion of module can be computed in polynomial time using a module extraction algorithm as an oracle.

Module extraction has been identified as a key task to support knowledge reuse (Cuenca Grau et al., 2008; Jiménez-Ruiz et al., 2008). Modules have also been exploited to optimise ontology matching (Jiménez-Ruiz \& Cuenca Grau, 2011) and the computation of justifications (Suntisrivaraporn et al., 2008; Ludwig, 2014) for ontology debugging and explanation. Finally, module extraction techniques have been applied to optimising ontology classification (Armas Romero et al., 2012; Tsarkov \& Palmisano, 2012; Suntisrivaraporn, 2008; Cuenca Grau et al., 2010) and have been integrated in the reasoners MORE and CHAINsaw. 


\subsection{Related Problems}

Module extraction is strongly related to the notions of forgetting and (uniform) interpolation (Eiter, Ianni, Schindlauer, Tompits, \& Wang, 2006; Ludwig \& Konev, 2014; Koopmann \& Schmidt, 2014; Konev, Walther, \& Wolter, 2009; Nikitina \& Rudolph, 2014; Wang, Wang, Topor, \& Pan, 2010). A uniform interpolant of an $\mathcal{L}$-ontology $\mathcal{O}$ and a signature $\Sigma$ is an $\mathcal{L}$-ontology $\mathcal{O}^{\prime}$ that only mentions symbols from $\Sigma$ and which is inseparable from $\mathcal{O}$ w.r.t. $\Sigma$ for a given inseparability relation. In contrast to modules, uniform interpolants are not required to be subsets of $\mathcal{O}$ and they cannot contain any symbol outside $\Sigma$ (all remaining symbols are thus forgotten). The latter requirement implies that uniform interpolants for a given $\Sigma$ and $\mathcal{O}$ may not always exist (Konev et al., 2009; Lutz \& Wolter, 2011; Wang, Wang, Topor, Pan, \& Antoniou, 2014).

Amir and McIlraith (2005) investigated partition-based reasoning techniques in propositional and first-order logic, where the goal is to improve the efficiency of reasoning over a knowledge base by first dividing its axioms into related partitions. The topology of the partitions is described by means of a graph, where nodes represent partitions and an edge between two partitions is labelled with the symbols they have in common. Such graph structure is then exploited by a distributed message-passing algorithm, the correctness of which is ensured by Craig's interpolation theorem for first-order logic. Similar problems and reasoning techniques have been studied in the context of description logics by Konev, Lutz, Ponomaryov, and Wolter (2010) as well as by Schlicht and Stuckenschmidt (2009). The key concern in partition-based reasoning is to find a partitioning that exhibits a suitable balance between number of partitions, their size, and the number of common symbols between partitions in order to enable efficient distributed reasoning. In this setting, partitions do not necessarily capture the meaning of a given signature in the input knowledge base and are therefore fundamentally different from modules.

Konev, Ludwig, Walther, and Wolter (2012) studied the problem of computing the logical difference of ontologies $\mathcal{O}$ and $\mathcal{O}^{\prime}$ - that is, the set of of queries that receive different answers w.r.t. $\mathcal{O}$ and $\mathcal{O}^{\prime}$. Computing the logical difference (or a concise representation thereof) has been identified as a valuable resource for ontology versioning tasks (Jiménez-Ruiz, Cuenca Grau, Horrocks, \& Berlanga Llavori, 2011) and is closely related to inseparability checking; indeed, inseparable ontologies are those that have an empty difference.

Finally, Zhou et al. (2014) proposed a hybrid approach to ontology-based query answering where the bulk of the computation is delegated to a datalog reasoner. Given an ontology $\mathcal{O}$, dataset $\mathcal{D}$, query $q(\mathbf{x})$, and candidate answer tuple $\mathbf{c}$, a core technique in this approach is to compute fragments $\mathcal{O}^{\prime} \subseteq \mathcal{O}$ and $\mathcal{D}^{\prime} \subseteq \mathcal{D}$ such that $\mathcal{O} \cup \mathcal{D} \models q(\mathbf{c})$ iff $\mathcal{O}^{\prime} \cup \mathcal{D}^{\prime} \models q(\mathbf{c})$. Similarly to our modules, these fragments are computed by first strengthening $\mathcal{O}$ into a datalog program $\mathcal{P}$ and then exploiting the datalog reasoner to identify the axioms and facts responsible for the validity of $q(\mathbf{c})$. In contrast to query inseparable modules, however, the fragment $\mathcal{O}^{\prime} \cup \mathcal{D}^{\prime}$ is only guaranteed to preserve the fixed query $q(\mathbf{c})$ w.r.t. the fixed dataset $\mathcal{D}$, rather than all queries w.r.t. all datasets over a reference signature.

\section{Implementation and Evaluation}

In this section, we present our prototype module extraction system and discuss the results of our evaluation on a suite of real-world ontologies. 


\subsection{The PrisM System}

We have implemented a prototype system for module extraction in Java, called PRISM, which bundles RDFox as a black-box datalog reasoner (Motik et al., 2014). PRISM is available online under academic license. ${ }^{4}$

PRISM accepts as input an OWL 2 ontology $\mathcal{O}$, a signature $\Sigma$ and a parameter $\mathcal{S}$ that indicates the relevant inseparability relation. Our system currently supports the whole of OWL 2, with the only exception of datatypes; furthermore, it supports the inseparability relations $\mathcal{S} \in\{\mathrm{m}, \mathrm{q}, \mathrm{f}, \mathrm{i}, \mathrm{c}, \mathrm{wq}\}$ defined in Sections 6 and 7 .

PRISM computes an $\mathcal{S}$-module $\mathcal{M}_{\text {out }}$ of $\mathcal{O}$ w.r.t. $\Sigma$ according to the following steps.

1. Compute the normalisation norm $(\mathcal{O})$ of $\mathcal{O}$, where norm is the normalisation function defined by the rules in Figure 2 from Section 2 (see also Proposition 3). Then, apply the mapping $\pi$ from Figure 1 to obtain an equivalent set of rules $\mathcal{O}^{\prime}=\pi(\operatorname{norm}(\mathcal{O}))$.

2. Consider the $\mathcal{S}$-module setting $\chi_{\mathcal{S}}=\left(\theta^{\mathcal{S}}, \mathcal{D}_{0}^{\mathcal{S}}, \mathcal{D}_{r}^{\mathcal{S}}\right)$ for $\mathcal{O}^{\prime}$ and $\Sigma$ as defined in Section 6 (for $\mathcal{S} \in\{\mathrm{m}, \mathrm{q}, \mathrm{f}, \mathrm{i}\}$ ) or 7 (for $\mathcal{S} \in\{\mathrm{c}, \mathrm{wq}\}$ ). Then, compute the corresponding module $\mathcal{M}^{\chi \mathcal{S}}$ as follows:

(a) Construct the datalog program $\mathcal{P}^{\chi \mathcal{S}}$ as specified in Definition 19, and the datasets $\mathcal{D}_{0}^{\mathcal{S}}$ and $\mathcal{D}_{r}^{\mathcal{S}}$ from $\chi_{\mathcal{S}}$

(b) Compute the support $\operatorname{supp}\left(\chi_{\mathcal{S}}\right)$ by exploiting the result in Theorem 68. For this,

- compute the datalog program $\Delta\left(\mathcal{P}^{\chi_{\mathcal{S}}}\right)$ and the dataset $\Delta\left(\mathcal{D}_{0}^{\mathcal{S}}, \mathcal{D}_{r}^{\mathcal{S}}\right)$ using the transformation $\Delta$ defined in Theorem 68 ;

- construct the materialisation $M a t$ of $\Delta\left(\mathcal{P}^{\chi \mathcal{S}}\right) \cup \Delta\left(\mathcal{D}_{0}^{\mathcal{S}}, \mathcal{D}_{r}^{\mathcal{S}}\right)$ using RDFox; and

- $\operatorname{construct} \operatorname{supp}\left(\chi_{\mathcal{S}}\right)$ as the set of rules $r \in \mathcal{P}^{\chi_{\mathcal{S}}}$ such that $\operatorname{Rel}\left(d_{r}\right) \in$ Mat

(c) Construct $\mathcal{M}^{\chi \mathcal{S}}$ from $\operatorname{supp}\left(\chi_{\mathcal{S}}\right)$ as specified in Definition 19.

3. Return $\mathcal{M}_{\text {out }}$ consisting of all axioms $\alpha \in \mathcal{O}$ such that $\pi(\operatorname{norm}(\alpha)) \cap \mathcal{M}^{\chi \mathcal{S}} \neq \emptyset$.

The first step normalises the input OWL 2 ontology $\mathcal{O}$ into a set of rules $\mathcal{O}^{\prime}$. PRISM provides an optimisation where $\mathcal{O}^{\prime}$ is constructed from a locality-based module of $\mathcal{O}$, rather than from $\mathcal{O}$ itself. Specifically, we use the $\perp$-module $\mathcal{M}_{[\mathcal{O}, \Sigma]}^{\perp}$ if $\mathcal{S}$ is classification inseparability, and the $\perp \top^{*}$-module $\mathcal{M}_{[\mathcal{O}, \Sigma]}^{\perp T^{*}}$ for all other inseparability relations. This optimisation does not compromise the correctness of the overall procedure since model inseparability is stronger than all other inseparability relations.

The second step computes the relevant $\mathcal{S}$-module $\mathcal{M}^{\chi \mathcal{S}}$ for the set of rules $\mathcal{O}^{\prime}$ and signature $\Sigma$. This is essentially done by following Definition 19; the only computationally demanding part is the construction of the $\operatorname{support} \operatorname{supp}\left(\chi_{\mathcal{S}}\right)$, which is achieved via materialisation using RDFOx in a black-box manner.

Finally, the third step constructs the module $\mathcal{M}_{\text {out }}$ for the input ontology $\mathcal{O}$ from the module $\mathcal{M}^{\chi \mathcal{S}}$ for its corresponding set of rules $\mathcal{O}^{\prime}$. For this, PRISM keeps track of the correspondence between the axioms in $\mathcal{O}$ and those in its normalisation norm $(\mathcal{O})$. Correctness of this step is ensured by Proposition 13.

4. http://www.cs.ox.ac.uk/isg/tools/PrisM/ 


\begin{tabular}{|c|c|c|c|c|c|c|}
\hline ID & name & predicates & rules & disjunctive & existential & expressivity \\
\hline 00001 & ACGT-v1.0 & 2,019 & 5,512 & 105 & 259 & $\overline{S \mathcal{R O I} \mathcal{Q}(D)}$ \\
\hline 00004 & BAMS-simplified & 1,199 & 18,976 & 0 & 16,782 & $\mathcal{A} \mathcal{L} \mathcal{E} \mathcal{H I F}^{+}$ \\
\hline 00024 & DOLCE & 603 & 2,148 & 53 & 184 & $\mathcal{S H O I N}(D)$ \\
\hline 00026 & GALEN-no-FIT & 29,073 & 66,191 & 0 & 26,973 & $\mathcal{A L E \mathcal { H }}$ \\
\hline 00029 & GALEN-doctored & 3,740 & 7,447 & 0 & 2,367 & $\mathcal{A L E \mathcal { H } \mathcal { I } \mathcal { F } ^ { + }}$ \\
\hline 00032 & GALEN-undoctored & 3,762 & 7,818 & 0 & 2,715 & $\mathcal{A L E \mathcal { H } \mathcal { I F } ^ { + }}$ \\
\hline 00347 & LUBM-one-uni & 68 & 84,771 & 0 & 8 & $\mathcal{A} \mathcal{L} \mathcal{E} \mathcal{H} \mathcal{I}^{+}(D)$ \\
\hline 00350 & OBI & 2,965 & 10,952 & 77 & 1,168 & $\mathcal{S H O I N}(D)$ \\
\hline 00351 & AERO & 355 & 669 & 11 & 100 & $\mathcal{S R O I} \mathcal{Q}(D)$ \\
\hline 00354 & NIF-gross-anatomy & 4,166 & 7,134 & 51 & 1,506 & $\mathcal{S R O \mathcal { I F } ( D )}$ \\
\hline 00463 & Fly-anatomy-XP & 8,047 & 42,107 & 0 & 9,433 & $\mathcal{A L E R \mathcal { I } ^ { + }}$ \\
\hline 00471 & FMA-lite & 78,986 & 168,828 & 0 & 42,734 & $\mathcal{A L E \mathcal { H } ^ { + }}$ \\
\hline 00477 & Gazetteer & 150,981 & 382,158 & 0 & 156,743 & $\mathcal{A L E} \mathcal{E}^{+}$ \\
\hline 00512 & Lipid & 1,289 & 5,222 & 541 & 893 & $\mathcal{A L C H \mathcal { N } N}$ \\
\hline 00545 & Molecule-role & 9,222 & 153,020 & 0 & 6,276 & $\mathcal{A L \mathcal { E } ^ { + }}$ \\
\hline 00774 & RNA-v0.2 & 338 & 938 & 34 & 90 & $\mathcal{S R I} \mathcal{Q}(D)$ \\
\hline 00775 & Roberts-family & 183 & 2,020 & 1 & 73 & $\mathcal{S R O \mathcal { I } Q ( D )}$ \\
\hline 00778 & SNOMED & 54,982 & 191,891 & 18,323 & 60,377 & $\mathcal{S H}$ \\
\hline 00786 & NCI-v12.04e & 93,628 & 193,453 & 65 & 76,957 & $\mathcal{S H}(D)$ \\
\hline
\end{tabular}

Table 3: Test ontologies

\subsection{Evaluation}

We have evaluated our system on a set of test ontologies identified in the work of Glimm, Horrocks, Motik, Stoilos, and Wang (2014) as non-trivial for reasoning. All ontologies have been normalised prior to module extraction to make DL axioms equivalent to rules. Further details on these ontologies are given in Table $3 .^{5}$ The first and second columns in the table indicate the ontology ID and name in the Oxford Ontology Repository. The third and fourth columns provide the number of predicates and rules in the resulting ontology after normalisation. The fifth and sixth columns specify how many of these rules contain disjunction and existential quantification in the head. Finally, the last column indicates the DL expressivity ${ }^{6}$ of the normalised ontology as given by the the OWL API.

All experiments were performed on a server with 2 Intel Xeon E5-2670 2.60GHz processors, each of which has 8 physical cores that serve 2 virtual cores each, making a total of 32 virtual cores. In our experiments we allocated 90GB of RAM, and RDFox was always run on 16 threads. We have compared the module sizes and extraction times using our system with those for locality-based $\perp$ - and $\perp T^{*}$-modules, computed using the OWL API. We have followed the experimental methodology in the work of Del Vescovo et al. (2013), where two kinds of signatures are considered:

- genuine signatures, which correspond to the signature of individual axioms, and

- random signatures, which include the signatures of several axioms.

5. The ontologies used in our experiments are available for download at https://krr-nas.cs.ox.ac.uk/ 2015/jair/PrisM/testOntologies.zip.

6. We refer the reader to the work of Baader et al. (2003) for a detailed account on DL naming conventions. 
Unlike the work of Del Vescovo et al. (2013), who defined random signatures simply as random subsets of the ontology signature, we extracted such signatures using a randomised graph sampling algorithm. We first represented the syntactic dependencies between symbols in the (normalised) ontology as a graph, and then traversed the graph in a randomised way until we visited a set number $n$ of nodes. The symbols corresponding to the visited nodes were then taken as a random signature. ${ }^{7}$ The advantage of this approach is that it yields signatures that are "semantically connected", which we believe is likely to be the case in practical applications. The number $n$ was chosen by default as $0.1 \%$ of the total graph and then increased by up to two orders of magnitude in cases where the resulting signatures typically contained less than 15 predicates and thus were too small to provide additional information w.r.t. genuine signatures.

For each kind of signature and each ontology, we have considered a sample of 400 runs and averaged module sizes and module extraction times. On the one hand, we have compared the modules produced by $\chi_{\mathrm{c}}$ (Section 7 ) with $\perp$-modules. which are the only kind of modules in the literature that guarantee our notion of classification inseparability. On the other hand, we have compared the modules produced by $\chi_{\mathrm{m}}, \chi_{\mathrm{q}}, \chi_{\mathrm{wq}}, \chi_{\mathrm{f}}$, and $\chi_{\mathrm{i}}$ (Sections 6 and 7) with $\perp T^{*}$-modules. As discussed in Section 12.2, no other system is (to the best of our knowledge) capable of computing modules specific to the deductive inseparability relations considered in this paper. Furthermore, other module extraction systems that ensure model inseparability, such as MEX and AMEX, are only applicable to rather restricted ontology languages. Consequently, $\perp T^{*}$-modules seemed the best available option for comparison to our approach.

Tables 4 and 5 provide the average number of rules in each kind of module for genuine and random signatures, respectively. In both tables, the total number of rules in the normalised ontology is provided at the top for comparison purposes, whereas the average size of the signatures considered is specified towards the bottom. Table 5 additionally includes the percentage $n$ of the dependency graph covered by the random walks from which random signatures were obtained.

We can observe that module size consistently decreases as we consider weaker inseparability relations. The modules produced by $\chi_{\mathrm{c}}$ can be several orders of magnitude smaller than $\perp$-modules, as in the cases of $00463,00471,00477$ or 00545 . Although those are rather extreme cases, we observed in most cases at least a 25\% decrease in size (see 00026, 00029, 00032, 00347, 00350, 00351, 00786). Our modules for model inseparability improve reasonably on $\perp T^{*}$-modules in most cases, although the greatest difference in size is of course between $\perp T^{*}$-modules and $\chi_{\mathbf{i}}$-modules, reaching one order of magnitude for some ontologies (see 00471 and 00477 , and also 0004, 00463 and 00786 with genuine signatures). In realistic ontologies, only a very small proportion of predicate pairs are related by atomic implication, and this is often still the case when considering a datalog overestimation of the ontology; thus, the difference in size between $\perp T^{*}$-modules and $\chi_{i}$ is rather unsurprising. There is naturally also a big difference in size between $\chi_{\text {wq }}$-modules and all other modules whenever ontologies do not mention any constants, since the former are in that case obviously empty. It is worth observing that, even though there are several cases where $\chi_{\mathrm{m}}$ modules and $\chi_{\mathrm{q}}$ modules are of very similar size (e.g. 00471), there are also cases where they differ signifi-

7. The functionality required to perform random walks is currently integrated in RDFox. 


\begin{tabular}{|c|r|r|r|r|r|r|r|r|r|r|}
\cline { 2 - 11 } \multicolumn{1}{c|}{} & 00001 & 00004 & 00024 & 00026 & 00029 & 00032 & 00347 & 00350 & 00351 & 00354 \\
\hline total & 5,512 & 18,976 & 2,148 & 66,191 & 7,447 & 7,818 & 84,771 & 10,952 & 669 & 7,134 \\
\hline$\perp$ & 678 & 18,306 & 1,000 & 14,253 & 187 & 690 & 84,726 & 803 & 133 & 826 \\
$\chi_{\mathrm{c}}$ & 558 & 16,942 & 883 & 9,799 & 94 & 479 & 23,651 & 558 & 74 & 618 \\
\hline $\mathcal{T}^{*}$ & 674 & 18,297 & 990 & 13,749 & 114 & 596 & 58,186 & 768 & 130 & 786 \\
$\chi_{\mathrm{m}}$ & 584 & 18,297 & 910 & 13,686 & 112 & 592 & 44,244 & 624 & 97 & 675 \\
$\chi_{\mathrm{q}}$ & 563 & 17,151 & 884 & 9,448 & 96 & 533 & 44,368 & 596 & 77 & 626 \\
$\chi_{\text {wq }}$ & 514 & 0 & 875 & 0 & 0 & 0 & 43,761 & 538 & 64 & 111 \\
$\chi_{\mathrm{f}}$ & 563 & 17,108 & 884 & 5,962 & 96 & 533 & 31,322 & 596 & 77 & 626 \\
$\chi_{\mathrm{i}}$ & 558 & 655 & 882 & 3,279 & 18 & 130 & 11,234 & 558 & 67 & 617 \\
\hline$|\Sigma|$ & 2 & 3 & 2 & 3 & 3 & 3 & 2 & 2 & 2 & 2 \\
\hline
\end{tabular}

\begin{tabular}{|c|r|r|r|r|r|r|r|r|r|}
\cline { 2 - 10 } \multicolumn{1}{c|}{} & 00463 & 00471 & 00477 & 00512 & 00545 & 00774 & 00775 & 00778 & 00786 \\
\hline total & 42,107 & 168,828 & 382,158 & 5,222 & 153,020 & 938 & 2,020 & 191,891 & 193,453 \\
\hline$\perp$ & 22,348 & 47,192 & 214,820 & 261 & 143,399 & 80 & 1,916 & 433 & 1,140 \\
$\chi_{\mathrm{c}}$ & 112 & 12 & $<1$ & 86 & 6 & 76 & 1,491 & 426 & 390 \\
\hline $\mathrm{T}^{*}$ & 221 & 20 & 9 & 34 & 2 & 80 & 1,913 & 427 & 1,138 \\
$\chi_{\mathrm{m}}$ & 217 & 12 & 8 & 32 & 1 & 80 & 1,498 & 426 & 1,138 \\
$\chi_{\mathrm{q}}$ & 107 & 12 & 8 & 29 & 1 & 78 & 1,492 & 426 & 385 \\
$\chi_{\mathrm{wq}}$ & 0 & 0 & 0 & 0 & 0 & 0 & 1,490 & 0 & 0 \\
$\chi_{\mathrm{f}}$ & 80 & 1 & $<1$ & 29 & $<1$ & 78 & 1,492 & 426 & 371 \\
$\chi_{\mathrm{i}}$ & 12 & 1 & $<1$ & 27 & $<1$ & 76 & 1,491 & 397 & 120 \\
\hline$|\Sigma|$ & 3 & 2 & 3 & 2 & 3 & 2 & 2 & 3 & 3 \\
\hline
\end{tabular}

Table 4: Average module sizes for genuine signatures.

cantly (e.g. 00786). Similarly, $\chi_{\mathrm{q}}$ modules and $\chi_{\mathrm{f}}$ modules have similar size in some cases (e.g. 00350) but not in others (e.g. 00471), and the same happens with $\chi_{\mathrm{q}}$ and $\chi_{\mathrm{wq}}$ (exemplified by ontologies 00775 and 00354), and with $\chi_{\mathrm{f}}$ and $\chi_{\mathrm{i}}$ (see ontologies 00512 and 00004). These observations suggest that our modules faithfully reflect the differences between the inseparability relations we considered, and that they could offer significant advantages for practical applications

Tables 6 and 7 provide the average module extraction time (in milliseconds) for genuine and random signatures, respectively. The extraction of our modules is consistently slower than that of locality-based modules; however, the average extraction time rarely exceeds 1 minute, and is very often below 10 seconds (especially for genuine signatures). This suggests that our modules are feasible for practical applications. Furthermore, since most of the extraction time is invariably spent by the datalog reasoner, which is used as a black box, future advancements in the area of datalog reasoning can lead to further performance gains for systems implementing our technique.

\section{Conclusion and Future Work}

In this paper, we have proposed a novel approach to module extraction based on a reduction to datalog reasoning. In contrast to existing techniques, our approach is not only applicable to description logics, but also to highly expressive first-order rule formalisms. Furthermore, our techniques can be easily customised so as to capture a wide range of inseparability relations studied in the literature. In all cases our modules satisfy many desirable properties, 


\begin{tabular}{|c|r|r|r|r|r|r|r|r|r|r|}
\cline { 2 - 12 } \multicolumn{1}{c|}{} & 00001 & 00004 & 00024 & 00026 & 00029 & 00032 & 00347 & 00350 & 00351 & 00354 \\
\hline total & 5,512 & 18,976 & 2,148 & 66,191 & 7,447 & 7,818 & 84,771 & 10,952 & 669 & 7,134 \\
\hline$\perp$ & 857 & 18,904 & 1,053 & 27,771 & 1,890 & 3,279 & 84,732 & 1,795 & 315 & 1,537 \\
$\chi_{\mathrm{c}}$ & 691 & 17,607 & 933 & 17,879 & 1,223 & 2,483 & 58,203 & 1,084 & 208 & 1,240 \\
\hline $\mathcal{T}^{*}$ & 854 & 18,894 & 1,044 & 27,184 & 1,726 & 3,108 & 80,153 & 1,758 & 311 & 1,501 \\
$\chi_{\mathrm{m}}$ & 759 & 18,894 & 964 & 27,175 & 1,719 & 3,101 & 63,031 & 1,611 & 274 & 1,388 \\
$\chi_{\mathrm{q}}$ & 736 & 18,579 & 942 & 18,315 & 1,380 & 2,633 & 63,031 & 1,389 & 265 & 1,279 \\
$\chi_{\text {wq }}$ & 517 & 0 & 875 & 0 & 0 & 0 & 62,455 & 539 & 81 & 113 \\
$\chi_{\mathrm{f}}$ & 735 & 18,536 & 942 & 18,255 & 1,364 & 2,620 & 58,980 & 1,389 & 241 & 1,278 \\
$\chi_{\mathrm{i}}$ & 688 & 2,511 & 931 & 17,646 & 1,060 & 2,314 & 50,362 & 1,080 & 191 & 1,238 \\
\hline$|\Sigma|$ & 43 & 82 & 20 & 107 & 104 & 107 & 11 & 92 & 56 & 79 \\
$\%$ & 1 & 1 & 1 & 0.1 & 1 & 1 & 10 & 1 & 10 & 1 \\
\hline
\end{tabular}

\begin{tabular}{|c|r|r|r|r|r|r|r|r|r|}
\cline { 2 - 10 } \multicolumn{1}{c|}{} & 00463 & 00471 & 00477 & 00512 & 00545 & 00774 & 00775 & 00778 & 00786 \\
\hline total & 42,107 & 168,828 & 382,158 & 5,222 & 153,020 & 938 & 2,020 & 191,891 & 193,453 \\
\hline$\perp$ & 23,139 & 49,345 & 215,886 & 1,555 & 143,448 & 371 & 1,979 & 11,766 & 16,820 \\
$\chi_{\mathrm{c}}$ & 595 & 402 & 38 & 1,199 & 28 & 338 & 1,527 & 11,342 & 7,974 \\
\hline $\mathcal{T}^{*}$ & 982 & 1,658 & 1,050 & 837 & 16 & 371 & 1,977 & 11,762 & 16,817 \\
$\chi_{\mathrm{m}}$ & 973 & 1,450 & 1,049 & 819 & 14 & 369 & 1,561 & 11,651 & 16,817 \\
$\chi_{\mathrm{q}}$ & 757 & 1,450 & 1,049 & 774 & 14 & 368 & 1,557 & 11,644 & 8,969 \\
$\chi_{\text {wq }}$ & 0 & 0 & 0 & 0 & 0 & 0 & 1,506 & 0 & 0 \\
$\chi_{\mathrm{f}}$ & 664 & 74 & 16 & 766 & 5 & 368 & 1,557 & 11,342 & 8,415 \\
$\chi_{\mathrm{i}}$ & 333 & 74 & 16 & 467 & 5 & 338 & 1,526 & 11,342 & 6,228 \\
\hline$|\Sigma|$ & 28 & 154 & 312 & 66 & 19 & 58 & 42 & 202 & 326 \\
$\%$ & 0.1 & 0.1 & 0.1 & 1 & 0.1 & 10 & 10 & 0.1 & 0.1 \\
\hline
\end{tabular}

Table 5: Average module sizes for random signatures.

which makes them well-suited to applications such as ontology reuse, debugging, modular ontology development, and reasoning optimisation. Last, but not least, our modules can be efficiently computed by reusing off-the-shelf datalog reasoners and our experimental evaluation confirms their suitability in practice.

We envisage many directions for future work, which we outline next.

- State-of-the-art modular DL reasoners, such as MORE and Chainsaw, currently rely on $\perp$-modules to split the workload between a fully-fledged OWL reasoner and an efficient reasoner for a lightweight DL. It would be natural to exploit our modules for classification inseparability (cf. Section 7.1) instead of $\perp$-modules since all such reasoners focus mainly on classification tasks. We believe that using our modules for modular reasoning will significantly improve the separation of workload and lead to a better use of the lightweight reasoner.

- So far, the use of modules for optimising data reasoning tasks, such as fact entailment and query answering, has been rather limited. Indeed, it is well-known that $\perp$-modules are not well-suited for such tasks (Cuenca Grau et al., 2008). PAGODA is the only reasoning system we know of that exploits techniques akin to module extraction for data reasoning (Zhou et al., 2014, 2015). It would be interesting to investigate how our techniques could be exploited to improve PAGODA's performance. Furthermore, 


\begin{tabular}{|c|r|r|r|r|r|r|r|r|r|r|}
\cline { 2 - 11 } \multicolumn{1}{c|}{} & 00001 & 00004 & 00024 & 00026 & 00029 & 00032 & 00347 & 00350 & 00351 & 00354 \\
\hline$\perp$ & 27 & 64 & 11 & 273 & 24 & 27 & 274 & 95 & 3 & 31 \\
$\chi_{\mathrm{c}}$ & 846 & 36,784 & 1,066 & 30,256 & 295 & 989 & 14,823 & 747 & 130 & 6,588 \\
\hline $\mathrm{T} \perp^{*}$ & 43 & 95 & 19 & 418 & 39 & 42 & 463 & 124 & 5 & 43 \\
$\chi_{\mathrm{m}}$ & 831 & 18,244 & 1,013 & 25,884 & 231 & 826 & 15,268 & 724 & 126 & 722 \\
$\chi_{\mathrm{q}}$ & 857 & 62,680 & 1,074 & 29,051 & 241 & 903 & 14,688 & 798 & 136 & 3,530 \\
$\chi_{\text {wq }}$ & 857 & 32,972 & 1,069 & 27,272 & 229 & 861 & 10,677 & 787 & 140 & 767 \\
$\chi_{\mathrm{f}}$ & 846 & 62,797 & 1,074 & 28,254 & 246 & 910 & 14,495 & 784 & 131 & 3,840 \\
$\chi_{\mathrm{i}}$ & 847 & 35,158 & 1,072 & 28,499 & 234 & 890 & 10,285 & 789 & 132 & 3,826 \\
\hline
\end{tabular}

\begin{tabular}{|c|r|r|r|r|r|r|r|r|r|}
\cline { 2 - 10 } \multicolumn{1}{c|}{} & 00463 & 00471 & 00477 & 00512 & 00545 & 00774 & 00775 & 00778 & 00786 \\
\hline$\perp$ & 122 & 506 & 1,154 & 18 & 363 & 5 & 10 & 722 & 569 \\
$\chi_{\mathrm{c}}$ & 4,176 & 9,729 & 52,529 & 378 & 34,705 & 161 & 963 & 1,657 & 3,449 \\
\hline $\mathrm{T}^{*}$ & 199 & 805 & 1,851 & 30 & 616 & 11 & 18 & 1,056 & 899 \\
$\chi_{\mathrm{m}}$ & 775 & 1,543 & 4,995 & 166 & 2,783 & 147 & 857 & 1,590 & 3,340 \\
$\chi_{\mathrm{q}}$ & 485 & 824 & 1,792 & 225 & 595 & 161 & 955 & 1,708 & 3,475 \\
$\chi_{\mathrm{wq}}$ & 455 & 790 & 1,753 & 210 & 552 & 154 & 966 & 1,612 & 3,419 \\
$\chi_{\mathrm{f}}$ & 463 & 788 & 1,772 & 229 & 580 & 164 & 967 & 1,700 & 3,526 \\
$\chi_{\mathrm{i}}$ & 456 & 792 & 1,759 & 229 & 579 & 164 & 965 & 1,691 & 3,479 \\
\hline
\end{tabular}

Table 6: Average extraction times in milliseconds for genuine signatures.

\begin{tabular}{|c|r|r|r|r|r|r|r|r|r|r|}
\cline { 2 - 11 } \multicolumn{1}{c|}{} & 00001 & 00004 & 00024 & 00026 & 00029 & 00032 & 00347 & 00350 & 00351 & 00354 \\
\hline$\perp$ & 26 & 58 & 10 & 284 & 35 & 39 & 276 & 107 & 4 & 29 \\
$\chi_{\mathrm{c}}$ & 1,076 & 36,376 & 1,162 & 56,125 & 2,989 & 5,737 & 15,708 & 2,065 & 423 & 13,664 \\
\hline$T \perp^{*}$ & 101 & 84 & 18 & 457 & 54 & 60 & 485 & 144 & 9 & 45 \\
$\chi_{\mathrm{m}}$ & 1,011 & 18,114 & 1,077 & 49,792 & 2,675 & 4,900 & 20,885 & 1,936 & 384 & 1,467 \\
$\chi_{\mathrm{q}}$ & 1,064 & 171,497 & 1,132 & 55,343 & 2,814 & 5,533 & 20,654 & 2,077 & 423 & 8,591 \\
$\chi_{\text {wq }}$ & 1,056 & 32,474 & 1,153 & 51,342 & 2,670 & 5,170 & 15,390 & 2,010 & 390 & 1,591 \\
$\chi_{\mathrm{f}}$ & 1,058 & 179,759 & 1,122 & 54,854 & 2,785 & 5,477 & 20,822 & 2,067 & 417 & 8,358 \\
$\chi_{\mathrm{i}}$ & 1,078 & 34,866 & 1,144 & 54,068 & 2,765 & 5,368 & 15,063 & 2,108 & 427 & 8,376 \\
\hline
\end{tabular}

\begin{tabular}{|c|r|r|r|r|r|r|r|r|r|}
\cline { 2 - 10 } \multicolumn{1}{c|}{} & 00463 & 00471 & 00477 & 00512 & 00545 & 00774 & 00775 & 00778 & 00786 \\
\hline$\perp$ & 138 & 549 & 1,172 & 23 & 342 & 5 & 10 & 841 & 698 \\
$\chi_{\mathrm{c}}$ & 5,441 & 13,291 & 51,707 & 20,712 & 31,616 & 768 & 1,064 & 34,980 & 44,463 \\
\hline $\mathrm{T} \perp^{*}$ & 192 & 793 & 1,768 & 37 & 576 & 10 & 20 & 1,210 & 1,070 \\
$\chi_{\mathrm{m}}$ & 1,615 & 3,202 & 6,073 & 2,188 & 2,504 & 640 & 967 & 20,409 & 41,283 \\
$\chi_{\mathrm{q}}$ & 1,431 & 2,669 & 3,191 & 2,939 & 591 & 750 & 1,046 & 34,330 & 43,785 \\
$\chi_{\text {wq }}$ & 1,332 & 2,638 & 3,157 & 2,756 & 567 & 715 & 1,028 & 20,293 & 43,007 \\
$\chi_{\mathrm{f}}$ & 1,391 & 2,640 & 3,157 & 2,964 & 569 & 748 & 1,047 & 33,565 & 44,604 \\
$\chi_{\mathrm{i}}$ & 1,382 & 2,664 & 3,223 & 2,891 & 562 & 767 & 1,048 & 34,774 & 44,168 \\
\hline
\end{tabular}

Table 7: Average extraction times in milliseconds for random signatures.

we also envision potential applications to incremental and stream reasoning, where data is frequently changing but queries and ontologies can be seen as fixed.

- Our conjecture that optimal module setting families for fact and query inseparability incur an exponential blowup w.r.t. the ones that we have chosen remains open. 
- The use of $\perp$-modules to exploit debugging and explanation systems for DL ontologies has proved rather successful (Suntisrivaraporn et al., 2008). Given that our modules are also justification-preserving, it would be interesting to evaluate the effectiveness of our modules for implication inseparability in this setting.

- Finally, the use of module extraction techniques has so far been largely restricted to description logics. Our techniques are, however, widely applicable and could be exploited in a number of reasoning tasks for ontology languages such as datalog ${ }^{ \pm}$and datalog ${ }^{ \pm, \vee}$, which are currently gaining momentum.

\section{Acknowledgments}

This paper is an extended version of our conference publication (Armas Romero, Kaminski, Cuenca Grau, \& Horrocks, 2015). This work has been supported by the Royal Society under a University Research Fellowship, by the EPSRC projects Score!, MaSI ${ }^{3}$, and DBOnto, and by the EU FP7 project Optique. We would like to thank the anonymous referees for their valuable comments and suggestions.

\section{Appendix A. Proofs for Section 11}

Theorem 73. The family $\Psi^{\mathcal{S}}$ is $\mathcal{S}$-optimal for $\mathcal{S} \in\{\mathrm{m}, \mathrm{i}, \mathrm{c}, \mathrm{wq}\}$.

We prove the result for each $\mathcal{S} \in\{\mathrm{m}, \mathrm{i}, \mathrm{c}, \mathrm{wq}\}$ separately.

Theorem 75. $\Psi^{\mathrm{m}}$ is m-optimal.

Proof. Suppose $\Psi^{\mathrm{m}}$ is not m-optimal. Then there must be some m-admissible, uniform family $\Psi$ and some $\mathcal{O}$ and $\Sigma$ such that $\mathcal{M}^{\Psi^{\mathrm{m}}(\mathcal{O}, \Sigma)} \nsubseteq \mathcal{M}^{\Psi(\mathcal{O}, \Sigma)}$. Let $\Psi^{\mathrm{m}}(\mathcal{O}, \Sigma)=\left\langle\theta^{\mathrm{m}}, \mathcal{D}_{0}^{\mathrm{m}}, \mathcal{D}_{r}^{\mathrm{m}}\right\rangle$ and $\Psi(\mathcal{O}, \Sigma)=\left\langle\theta, \mathcal{D}_{0}, \mathcal{D}_{r}\right\rangle$. By Theorem 55, we have $\Psi^{\mathrm{m}}(\mathcal{O}, \Sigma) \hookrightarrow \Psi(\mathcal{O}, \Sigma)$, hence for each mapping $\mu: \operatorname{Ct}\left(\Psi^{\mathrm{m}}(\mathcal{O}, \Sigma)\right) \rightarrow \operatorname{Ct}(\Psi(\mathcal{O}, \Sigma))$ it must be either $\theta^{\mathrm{m}} \mu \neq \theta$ or $\mathcal{D}_{0}^{\mathrm{m}} \mu \nsubseteq \mathcal{D}_{0}$ or $\mathcal{D}_{r}^{\mathrm{m}} \mu \nsubseteq \mathcal{D}_{r}$.

Suppose $\theta$ is such that there are two existentially quantified variables $y_{1}$ and $y_{2}$ in $\mathcal{O}$ such that $y_{1} \theta \neq y_{2} \theta$. Consider the ontology $\mathcal{O}^{\prime}$ consisting of the following rules:

$$
\begin{array}{ll}
p_{1}: & A(x) \rightarrow S(x, b) \\
p_{2} & : A(x) \rightarrow \exists y_{1}\left[R\left(x, y_{1}\right) \wedge B\left(y_{1}\right)\right] \\
p_{3}: & A(x) \rightarrow \exists y_{2}\left[R\left(x, y_{2}\right) \wedge C\left(y_{2}\right)\right] \\
p_{4} & : \quad S(x, b) \wedge R(x, z) \wedge B(z) \wedge C(z) \rightarrow D(x)
\end{array}
$$

and the signature $\Sigma^{\prime}=\{A, D, R\}$ and let $\Psi\left(\mathcal{O}^{\prime}, \Sigma^{\prime}\right)=\left\langle\theta^{\prime}, \mathcal{D}_{0}^{\prime}, \mathcal{D}_{r}^{\prime}\right\rangle$. By the second property of uniformity, $\theta^{\prime}$ is the same as the substitution in $\Psi\left(\mathcal{O}^{\prime}, \emptyset\right)$ and $\Psi\left(\mathcal{O}^{\prime}, \Sigma\right)$, and therefore, by the first property of uniformity, $y_{1} \theta^{\prime} \neq y_{2} \theta^{\prime}$. It follows that $p_{4}$ can never be applied on $\mathcal{P}^{\Psi\left(\mathcal{O}^{\prime}, \Sigma^{\prime}\right)}\left(\mathcal{D}_{0}^{\prime}\right)$ and hence it is not in the support of $\Psi\left(\mathcal{O}^{\prime}, \Sigma^{\prime}\right)$ and $\mathcal{M}^{\Psi\left(\mathcal{O}^{\prime}, \Sigma^{\prime}\right)} \subseteq\left\{p_{1}, p_{2}, p_{3}\right\}$. The interpretation $\mathcal{I}$ such that $\Delta^{\mathcal{I}}=\{i\}, A^{\mathcal{I}}=B^{\mathcal{I}}=C^{\mathcal{I}}=\{i\}, D^{\mathcal{I}}=\emptyset, a^{\mathcal{I}}=i$ and $R^{\mathcal{I}}=S^{\mathcal{I}}=\{(i, i)\}$ is a model of $\mathcal{M}^{\Psi\left(\mathcal{O}^{\prime}, \Sigma^{\prime}\right)}$, but it cannot be extended to a model of $\mathcal{O}^{\prime}$ without changing the interpretation of $A, D$ or $R$. It follows that $\mathcal{M}^{\Psi\left(\mathcal{O}^{\prime}, \Sigma^{\prime}\right)}$ is not 
$\mathrm{a} \equiv \sum_{\Sigma^{\prime}}^{\mathrm{m}}$-module of $\mathcal{O}^{\prime}$, contradicting our hypothesis that $\Psi$ is m-admissible. The origin of this contradiction is in the assumption that $\theta$ does not map all existentially quantified variables to the same constant, therefore there must be some constant $c$ such that $y \theta=c$ for each variable $y$ universally quantified in $\mathcal{O}$. Suppose now that $\theta$ is such that there exists a constant $b^{\prime}$ in $\mathcal{O}$ such that $b^{\prime} \theta \neq c$. By the first property of uniformity $\theta^{\prime}$ must also not map $b$ to the same constant as $y_{1}$ and $y_{2}$. But then again $p_{4}$ can never be applied on $\mathcal{P}^{\Psi\left(\mathcal{O}^{\prime}, \Sigma^{\prime}\right)}\left(\mathcal{D}_{0}^{\prime}\right)$ and $\mathcal{M}^{\Psi\left(\mathcal{O}^{\prime}, \Sigma^{\prime}\right)} \subseteq\left\{p_{1}, p_{2}, p_{3}\right\}$, which, as we have already shown, is a contradiction. Consequently, $\theta$ must map all constants in $\mathcal{O}$ to $c$ as well.

This means that there exists some substitution $\mu: \operatorname{Ct}\left(\Psi^{\mathrm{m}}(\mathcal{O}, \Sigma)\right) \rightarrow \operatorname{Ct}(\Psi(\mathcal{O}, \Sigma))$ such that $\theta^{\mathrm{m}} \mu=\theta$. In particular it must be $* \mu=c$. By hypothesis, it must be the case that either $\mathcal{D}_{0}^{\mathrm{m}} \mu \nsubseteq \mathcal{D}_{0}$ or $\mathcal{D}_{r}^{\mathrm{m}} \mu \nsubseteq \mathcal{D}_{r}$.

Suppose $\mathcal{D}_{0}^{\mathrm{m}} \mu \nsubseteq \mathcal{D}_{0}$. There must be some predicate $X \in \Sigma$ such that $X(c, \ldots, c) \notin \mathcal{D}_{0}$. Consider the ontology $\mathcal{O}^{\prime \prime}$ consisting of the following rules:

$$
\begin{aligned}
& p_{5}: X(x, \ldots, x) \rightarrow \exists y R(x, y) \\
& p_{6}: R(x, x) \rightarrow A(x)
\end{aligned}
$$

and the signature $\Sigma^{\prime \prime}=\{X, A\}$, and let $\Psi\left(\mathcal{O}^{\prime \prime}, \Sigma^{\prime \prime}\right)=\left\langle\theta^{\prime \prime}, \mathcal{D}_{0}^{\prime \prime}, \mathcal{D}_{r}^{\prime \prime}\right\rangle$. By uniformity of $\Psi, \theta^{\prime \prime}$ maps $y$ to $c$, and the fact $X(c, \ldots, c)$ is not in the initial dataset of $\Psi(\mathcal{O},\{X\})$, or in that of $\Psi\left(\mathcal{O}, \Sigma^{\prime \prime}\right)$, or in $\mathcal{D}_{0}^{\prime \prime}$. Consider the dataset

$$
\hat{\mathcal{D}}=\{A(a), A(c)\} \cup\left\{X(\mathbf{t}) \mid \mathbf{t} \in\{a, c\}^{\operatorname{arity}(X)}, \mathbf{t} \neq(c, \ldots, c)\right\}
$$

with $a$ a fresh constant. The substitution $\hat{\mu}$ that maps $c$ to itself and all other constants in $\Psi\left(\mathcal{O}^{\prime \prime}, \Sigma^{\prime \prime}\right)$ to $a$ is a homomorphism from $\Psi\left(\mathcal{O}^{\prime \prime}, \Sigma^{\prime \prime}\right)$ to $\hat{\chi}=\left\langle\theta^{\prime \prime}, \hat{\mathcal{D}}, \Gamma_{\hat{\mu}}\left(\mathcal{D}_{r}^{\prime \prime}\right)\right\rangle$. By Theorem 55 , this implies $\mathcal{M}^{\Psi\left(\mathcal{O}^{\prime \prime}, \Sigma^{\prime \prime}\right)} \subseteq \mathcal{M}^{\hat{\chi}}$. Clearly, the only $R$-fact in $\mathcal{P} \hat{\chi}(\hat{\mathcal{D}})$ is $R(a, c)$, so $p_{5}$ is not in the support of $\hat{\chi}$ and therefore $\mathcal{M}^{\Psi\left(\mathcal{O}^{\prime \prime}, \Sigma^{\prime \prime}\right)} \subseteq \mathcal{M}^{\hat{\chi}} \subseteq\left\{p_{5}\right\}$. The interpretation $\mathcal{J}$ such that $\Delta^{\mathcal{J}}=\{i\}, X^{\mathcal{J}}=(i, \ldots, i), A^{\mathcal{J}}=\emptyset$ and $R^{\mathcal{J}}=\{(i, i)\}$ is a model of $\mathcal{M}^{\Psi\left(\mathcal{O}^{\prime}, \Sigma^{\prime \prime}\right)}$, but it cannot be extended to a model of $\mathcal{O}^{\prime \prime}$ without changing the interpretation of $X$ or $A$. It follows that $\mathcal{M}^{\Psi\left(\mathcal{O}^{\prime \prime}, \Sigma^{\prime \prime}\right)}$ is not a $\equiv_{\Sigma^{\prime \prime}}^{\mathrm{m}}$-module of $\mathcal{O}^{\prime \prime}$, which contradicts our hypothesis that $\Psi$ is m-admissible. This contradiction stems from the assumption that $\mathcal{D}_{0}^{\mathrm{m}} \mu \nsubseteq \mathcal{D}_{0}$, therefore it must be $\mathcal{D}_{0}^{\mathrm{m}} \mu \subseteq \mathcal{D}_{0}$.

By hypothesis, this implies $\mathcal{D}_{r}^{\mathrm{m}} \mu \nsubseteq \mathcal{D}_{r}$, so there must be some predicate $Y \in \Sigma$ such that $Y(c, \ldots, c) \notin \mathcal{D}_{r}$. Consider the ontology $\mathcal{O}^{\prime \prime \prime}$ consisting of the following rules:

$$
\begin{array}{ll}
p_{7}: & A(x) \rightarrow \exists y R(x, y) \\
p_{8}: & R(x, x) \rightarrow Y(x, \ldots, x)
\end{array}
$$

and the signature $\Sigma^{\prime \prime \prime}=\{A, Y\}$, and let $\Psi\left(\mathcal{O}^{\prime \prime \prime}, \Sigma^{\prime \prime \prime}\right)=\left\langle\theta^{\prime \prime \prime}, \mathcal{D}_{0}^{\prime \prime \prime}, \mathcal{D}_{r}^{\prime \prime \prime}\right\rangle$. By uniformity of $\Psi, \theta^{\prime \prime \prime}$ maps $y$ to $c$. Consider the dataset $\check{\mathcal{D}}=\{A(c), Y(c, \ldots, c), A(b), Y(b, \ldots, b)\}$, with $a$ again a fresh constant. The mapping $\check{\mu}$ that maps $c$ to itself and all other constants in $\Psi\left(\mathcal{O}^{\prime \prime \prime}, \Sigma^{\prime \prime \prime}\right)$ to $b$ is a homomorphism from $\Psi\left(\mathcal{O}^{\prime \prime \prime}, \Sigma^{\prime \prime \prime}\right)$ to $\check{\chi}=\left\langle\theta^{\prime \prime \prime}, \check{\mathcal{D}}, \mathcal{D}_{r}^{\prime \prime \prime} \check{\mu}\right\rangle$. By Theorem 55 , this implies $\mathcal{M}^{\Psi\left(\mathcal{O}^{\prime \prime \prime}, \Sigma^{\prime \prime}\right)} \subseteq \mathcal{M}^{\tilde{\chi}}$. It is easy to see that the only $R$-facts in the materialisation of $\mathcal{P} \check{\chi} \cup \check{\mathcal{D}}$ are $R(a, c)$ and $R(c, c)$, so the only proof in $\mathcal{P} \check{\chi} \cup \check{\mathcal{D}}$ supported by $p_{8}$ is a proof of $Y(c, \ldots, c)$. However, by uniformity of $\Psi, Y(c, \ldots, c)$ is not in the relevant facts of $\Psi(\mathcal{O},\{Y\})$, or in those of $\Psi(\mathcal{O},\{A, Y\})$, or in $\mathcal{D}_{r}^{\prime \prime \prime}$. It follows that $p_{8}$ is not in the support of $\check{\chi}$ and $\mathcal{M}^{\Psi\left(\mathcal{O}^{\prime \prime \prime}, \Sigma^{\prime \prime \prime}\right)} \subseteq \mathcal{M}^{\check{\chi}} \subseteq\left\{p_{7}\right\}$. The interpretation $\mathcal{K}$ such that $\Delta^{\mathcal{K}}=\{i\}, A^{\mathcal{K}}=\{i\}$, 
$R^{\mathcal{K}}=\{(i, i)\}$ and $Y^{\mathcal{K}}=\emptyset$ is a model of $\mathcal{M}^{\Psi\left(\mathcal{O}^{\prime}, \Sigma^{\prime \prime}\right)}$, but it cannot be extended to a model of $\mathcal{O}^{\prime \prime \prime}$ without changing the interpretation of $A$ or $Y$. It follows that $\mathcal{M}^{\Psi\left(\mathcal{O}^{\prime \prime \prime}, \Sigma^{\prime \prime \prime}\right)}$ is not a $\equiv_{\Sigma^{\prime \prime \prime}}^{\mathrm{m}}$-module of $\mathcal{O}^{\prime \prime \prime}$, which again contradicts our hypothesis that $\Psi$ is m-admissible. The origin of this contradiction is in the assumption that $\mathcal{D}_{r}^{\mathrm{m}} \mu \nsubseteq \mathcal{D}_{r}$, therefore it must be $\mathcal{D}_{r}^{\mathrm{m}} \mu \subseteq \mathcal{D}_{r}$. The mapping $\mu$ is thus a witness that $\Psi^{\mathrm{m}}(\mathcal{O}, \Sigma) \hookrightarrow \Psi(\mathcal{O}, \Sigma)$, ultimately contradicting the assumption that $\Psi^{\mathrm{m}}$ is not m-optimal.

Theorem 76. $\Psi^{\mathrm{i}}$ is i-optimal.

Proof. Consider the module setting family $\Psi_{0}^{\mathrm{i}}$ such that for each pair of ontology $\mathcal{O}$ and signature $\Sigma, \Psi_{0}^{\mathrm{i}}(\mathcal{O}, \Sigma)=\left\langle\theta^{\mathrm{i}_{0}}, \mathcal{D}_{0}^{\mathrm{i}_{0}}, \mathcal{D}_{r}^{\mathrm{i}_{0}}\right\rangle$ is as follows:

- $\theta^{i_{0}}=\left\{y \mapsto c_{y} \mid y\right.$ existentially quantified in $\left.\mathcal{O}\right\} \cup\{c \mapsto c \mid c \in \operatorname{Sig}(\mathcal{O})$ a constant $\}$

- $\mathcal{D}_{0}^{\mathrm{i}_{0}}=\left\{A\left(\mathbf{c}_{A, B}\right) \mid A \neq B\right.$ predicates in $\Sigma$ of the same arity $\}$

- $\mathcal{D}_{r}^{i_{0}}=\left\{B\left(\mathbf{c}_{A, B}\right) \mid A \neq B\right.$ predicates in $\Sigma$ of the same arity $\} \cup \mathcal{D}_{r_{\perp}}^{i_{0}}$

where each $c_{y}$ is a fresh constant, $\mathbf{c}_{A, B}=c_{A, B}^{1}, \ldots, c_{A, B}^{n}$, is an array of fresh constants for each pair $A, B \in \Sigma$ of distinct $n$-ary predicates, and $\mathcal{D}_{r_{\perp}}^{i_{0}}=\{\perp\}$ if $\Sigma$ contains two distinct predicates of the same arity and $\mathcal{D}_{r_{\perp}}^{i_{0}}=\emptyset$ otherwise.

First, we show that $\Psi^{\mathrm{i}}$ is i-optimal iff $\Psi_{0}^{\mathrm{i}}$ is i-optimal by proving that $\mathcal{M}^{\Psi^{\mathrm{i}}(\mathcal{O}, \Sigma)}=$ $\mathcal{M}^{\Psi_{0}^{\mathrm{i}}(\mathcal{O}, \Sigma)}$ for each $\mathcal{O}$ and $\Sigma$. Let us fix arbitrary $\mathcal{O}$ and $\Sigma$, and let $\Psi^{\mathrm{i}}(\mathcal{O}, \Sigma)=\left\langle\theta^{\mathrm{i}}, \mathcal{D}_{0}^{\mathrm{i}}, \mathcal{D}_{r}^{\mathrm{i}}\right\rangle$ and $\Psi_{0}^{\mathrm{i}}(\mathcal{O}, \Sigma)=\left\langle\theta^{\mathrm{i}_{0}}, \mathcal{D}_{0}^{\mathrm{i}_{0}}, \mathcal{D}_{r}^{\mathrm{i}_{0}}\right\rangle$. It is easy to see that $\Psi_{0}^{\mathrm{i}}(\mathcal{O}, \Sigma) \hookrightarrow \Psi^{\mathrm{i}}(\mathcal{O}, \Sigma)$, therefore by Theorem 55 we have that $\mathcal{M}^{\Psi_{0}^{\mathrm{i}}(\mathcal{O}, \Sigma)} \subseteq \mathcal{M}^{\Psi^{\mathrm{i}}(\mathcal{O}, \Sigma)}$. For $\mathcal{M}^{\Psi^{\mathrm{i}}(\mathcal{O}, \Sigma)} \subseteq \mathcal{M}^{\Psi_{0}^{\mathrm{i}}(\mathcal{O}, \Sigma)}$, first note that $\mathcal{P}^{\Psi^{\mathrm{i}}(\mathcal{O}, \Sigma)}=\mathcal{P}^{\Psi_{0}^{\mathrm{i}}(\mathcal{O}, \Sigma)}$. We can assume w.l.o.g. that $\Sigma$ contains at least two predicates $A, B$ of the same arity - otherwise there are no non-trivial $\Sigma$-implications. Given a proof $\rho=(T, \lambda)$ in $\mathcal{P}^{\Psi^{i}(\mathcal{O}, \Sigma)} \cup \mathcal{D}_{0}^{\mathrm{i}}$ of $B\left(\mathbf{c}_{A}\right)$ (resp. of $\perp$ ) the proof $\rho_{0}=\left(T, \lambda_{0}\right)$ such that $\lambda_{0}(v)=\lambda(v) \nu$ for each node $v$ in $T$, with $\nu$ a substitution such that $\mathbf{c}_{A} \nu=\mathbf{c}_{A, B}$, is a proof of $B\left(\mathbf{c}_{A, B}\right)$ (resp. of $\perp$ ) in $\mathcal{P}^{\Psi^{\mathrm{i}_{0}}(\mathcal{O}, \Sigma)} \cup \mathcal{D}_{0}^{\mathrm{i}_{0}}$ satisfying $\operatorname{supp}\left(\rho_{0}\right)=\operatorname{supp}(\rho)$. Consequently, $\operatorname{supp}\left(\Psi^{\mathrm{i}}(\mathcal{O}, \Sigma)\right) \subseteq \operatorname{supp}\left(\Psi_{0}^{\mathrm{i}}(\mathcal{O}, \Sigma)\right)$ and hence $\mathcal{M}^{\Psi^{\mathrm{i}}(\mathcal{O}, \Sigma)} \subseteq \mathcal{M}^{\Psi_{0}^{\mathrm{i}}(\mathcal{O}, \Sigma)}$.

Now, suppose $\Psi_{0}^{\mathrm{i}}$ is not $\mathrm{i}$-optimal. Then, there must be a uniform, i-admissible family $\Psi$ and some $\mathcal{O}$ and $\Sigma$ such that $\mathcal{M}^{\Psi_{0}^{\mathrm{i}}(\mathcal{O}, \Sigma)} \nsubseteq \mathcal{M}^{\Psi(\mathcal{O}, \Sigma)}$. Let $\Psi(\mathcal{O}, \Sigma)=\left\langle\theta, \mathcal{D}_{0}, \mathcal{D}_{r}\right\rangle$. Since $\theta^{\mathrm{i}_{0}}$ is injective, there exists a mapping $\mu: \operatorname{Ct}\left(\Psi_{0}^{\mathrm{i}}(\mathcal{O}, \Sigma)\right) \rightarrow \operatorname{Ct}(\Psi(\mathcal{O}, \Sigma))$ such that $\theta^{\mathrm{i}_{0}} \mu=\theta$. This condition only determines the effect of $\mu$ on $\operatorname{dom}\left(\theta^{i_{0}}\right)$, and since $\operatorname{dom}\left(\theta^{i_{0}}\right)$ is disjoint with the set $\left\{\mathbf{c}_{A, B} \mid A \neq B\right.$ predicates in $\Sigma$ of the same arity $\}$, we can asume that either $\mu$ is such that $\mathcal{D}_{0}^{i_{0}} \mu \subseteq \mathcal{D}_{0}$, or there are two distinct predicates $A, B \in \Sigma$ of the same arity such that $\mathcal{D}_{0}$ contains no $A$-facts. Suppose the latter is the case, and consider the ontology $\mathcal{O}^{\prime}=\{A(\mathbf{x}) \rightarrow B(\mathbf{x})\}$. By uniformity of $\Psi$, the initial dataset of $\Psi\left(\mathcal{O}^{\prime}, \Sigma\right)$ also contains no $A$-facts and therefore the support of $\Psi\left(\mathcal{O}^{\prime}, \Sigma\right)$ is empty and $\mathcal{M}^{\Psi\left(\mathcal{O}^{\prime}, \Sigma\right)}=\emptyset \not \models A(\mathbf{x}) \rightarrow B(\mathbf{x})$. This contradicts the i-admissibility of $\Psi$, hence it must be $\mathcal{D}_{0}^{\mathrm{i}_{0}} \mu \subseteq \mathcal{D}_{0}$. Finally, suppose $\mathcal{D}_{r}^{\mathrm{i}_{0}} \mu \nsubseteq \mathcal{D}_{r}$; then in particular $\Sigma$ must contain two distinct $n$-ary predicates, since otherwise it is $\mathcal{D}_{r}^{i_{0}}=\emptyset$ and thus trivially $\mathcal{D}_{r}^{i_{0}} \mu \nsubseteq \mathcal{D}_{r}$. In this case there are two possible situations:

- $\perp \notin \mathcal{D}_{r}$.

Let $A, B \in \Sigma$ be distinct predicates of the same arity and consider the ontology 
$\mathcal{O}^{\prime \prime}=\{A(\mathbf{x}) \rightarrow C(\mathbf{x}) \vee D(\mathbf{x}), C(\mathbf{x}) \rightarrow B(\mathbf{x}), D(\mathbf{x}) \rightarrow \perp\}$ with $C$ and $D$ fresh predicates. Clearly, $\mathcal{O}^{\prime \prime} \models A(\mathbf{x}) \rightarrow B(\mathbf{x})$ By uniformity of $\Psi, \perp$ is not in the set of relevant facts of $\Psi\left(\mathcal{O}^{\prime \prime}, \Sigma\right)$, and therefore $D(\mathbf{x}) \rightarrow \perp \notin \mathcal{M}^{\Psi\left(\mathcal{O}^{\prime \prime}, \Sigma\right)}$. Consequently we have $\mathcal{M}^{\Psi\left(\mathcal{O}^{\prime \prime}, \Sigma\right)} \not \models A(\mathbf{x}) \rightarrow B(\mathbf{x})$, contradicting the assumption that $\Psi$ is i-admissible.

- $B\left(\mathbf{c}_{A, B}\right) \mu \notin \mathcal{D}_{r}$ for some pair of distinct $A, B \in \Sigma$ of the same arity.

Consider $\mathcal{O}^{\prime \prime \prime}=\{A(\mathbf{x}) \rightarrow B(\mathbf{x})\}$. By uniformity of $\Psi$, there are no $B$-facts in the set of relevant facts of $\Psi\left(\mathcal{O}^{\prime \prime \prime},\{A, B\}\right)$, and therefore $\mathcal{M}^{\Psi\left(\mathcal{O}^{\prime \prime},\{A, B\}\right)}=\emptyset \not \vDash A(\mathbf{x}) \rightarrow B(\mathbf{x})$, contradicting the assumption that $\Psi$ is i-admissible.

It follows that $\mathcal{D}_{r}^{\mathrm{i}_{0}} \mu \subseteq \mathcal{D}_{r}$, so $\mu$ is a homomorphism from $\Psi(\mathcal{O}, \Sigma)$ to $\Psi_{0}^{\mathrm{i}}(\mathcal{O}, \Sigma)$, and thus $\mathcal{M}^{\Psi_{0}^{\mathrm{i}}(\mathcal{O}, \Sigma)} \subseteq \mathcal{M}^{\Psi(\mathcal{O}, \Sigma)}$, which ultimately contradicts the assumption that $\Psi_{0}^{\mathrm{i}}$ (resp. $\left.\Psi^{\mathrm{i}}\right)$ is not i-optimal.

Theorem 77. $\Psi^{\mathrm{c}}$ is c-optimal.

Proof. Analogous to Theorem 76

Theorem 78. $\Psi^{\mathrm{wq}}$ is wq-optimal.

Proof. Suppose $\Psi^{\mathrm{wq}}$ is not wq-optimal. Then there must be some uniform and wq-admissible family $\Psi$ and some $\mathcal{O}$ and $\Sigma$ s.t. $\mathcal{M}^{\Psi^{\mathrm{wq}}(\mathcal{O}, \Sigma)} \nsubseteq \mathcal{M}^{\Psi(\mathcal{O}, \Sigma)}$. Let $\Psi(\mathcal{O}, \Sigma)=\left\langle\theta, \mathcal{D}_{0}, \mathcal{D}_{r}\right\rangle$ and $\Psi^{\mathrm{wq}}(\mathcal{O}, \Sigma)=\left\langle\theta^{\mathrm{wq}}, \mathcal{D}_{0} \mathrm{wq}, \mathcal{D}_{r}^{\mathrm{wq}}\right\rangle$. By Theorem 55 , given a mapping $\mu: \operatorname{Ct}\left(\Psi^{\mathrm{wq}}\right) \rightarrow \operatorname{Ct}(\Psi)$ it must be either $\theta^{\mathrm{wq}} \mu \neq \theta$ or $\mathcal{D}_{0}^{\mathrm{wq}} \mu \nsubseteq \mathcal{D}_{0}$ or $\mathcal{D}_{r}^{\mathrm{wq}} \mu \nsubseteq \mathbb{\mathcal { D } _ { r }}$.

Since $\theta^{\mathrm{wq}}$ is injective and $\mathcal{D}^{\mathrm{wq}}=\emptyset$, we can assume that $\mu$ is such that $\theta^{\mathrm{wq}} \mu=\theta$, $\mathcal{D}_{0}^{\text {wq }} \mu \subseteq \mathcal{D}_{0}$ and $\mathcal{D}_{r}^{\text {wq }} \mu \nsubseteq \mathcal{D}_{r}$. But then there must be some predicate $X \in \Sigma$ and an array c of size $\operatorname{arity}(X)$ of constants from $\operatorname{Ct}(\mathcal{O}) \cup\left\{c_{y} \mid y\right.$ exist. quant. in $\left.\mathcal{O}\right\}$ such that $X(\mathbf{c}) \mu \notin \mathcal{D}_{r}$. Consider the ontology $\mathcal{O}^{\prime}=\{(\rightarrow X(\mathbf{c}))\}$; clearly, $\mathcal{O}^{\prime}=X(\mathbf{c})$. By uniformity, $X(\mathbf{c})$ is also not in the relevant facts of $\Psi\left(\mathcal{O}^{\prime}, \Sigma\right)$, which implies $\mathcal{M}^{\Psi\left(\mathcal{O}^{\prime}, \Sigma\right)}=\emptyset \not \models X(\mathbf{c})$. This contradicts the wq-admissibility of $\Psi$, hence it must be $\mathcal{D}_{r}^{\text {wq }} \mu \subseteq \mathcal{D}_{r}$, which makes $\mu$ a homomorphism. It follows that $\Psi^{\mathrm{wq}}$ is wq-optimal.

Theorem 74. The family $\Psi^{\mathcal{S}}$ is not $\mathcal{S}$-optimal for $\mathcal{S} \in\{\mathrm{f}, \mathrm{q}\}$.

Again, we prove the result for each $\mathcal{S} \in\{\mathrm{f}, \mathrm{q}\}$ separately.

Proposition 79. The family $\Psi^{\mathrm{f}}$ is not $\mathrm{f}$-optimal.

Proof. Consider an arbitrary but fixed pair of $\mathcal{O}$ and $\Sigma$. Let $\operatorname{Ct}(\mathcal{O})=\left\{c_{1}, \ldots, c_{n}\right\}$. Furthermore, for each predicate $B \in \Sigma$ and each array $\mathbf{v} \in\{1, \ldots \text {, arity }(B)+n\}^{\text {arity }(B)}$ consider a set of constants $\left\{*_{B, \mathbf{v}}^{i} \mid 0 \leq i \leq \operatorname{arity}(B)+n\right\}$ such that

1. $*_{B, \mathbf{v}}^{0}, \ldots, *_{B, \mathbf{v}}^{\text {arity }}(B)$ are fresh constants and

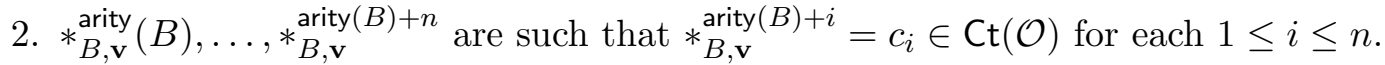

Let $*_{B, \mathbf{v}}^{\mathbf{w}}$ denote the array $\left(*_{B, \mathbf{v}}^{w_{1}}, \ldots, *_{B, \mathbf{v}}^{w_{n}}\right)$ whenever $\mathbf{w}=\left(w_{1}, \ldots, w_{n}\right)$, and consider the uniform module setting family $\Psi_{0}^{\mathrm{f}}$ such that $\Psi_{0}^{\mathrm{f}}(\mathcal{O}, \Sigma)=\left\langle\theta^{\mathrm{f}_{0}}, \mathcal{D}_{0}^{\mathrm{f}_{0}}, \mathcal{D}_{r}^{\mathrm{f}_{0}}\right\rangle$ is as follows:

- $\theta^{f_{0}}=\left\{y \mapsto c_{y} \mid y\right.$ existentially quantified in $\left.\mathcal{O}\right\} \cup\{c \mapsto c \mid c \in \operatorname{Ct}(\mathcal{O})\}$ 


$$
\begin{gathered}
\text { - } \mathcal{D}_{0}^{\mathrm{f}_{0}}=\left\{A\left(*_{B, \mathbf{v}}^{\mathbf{w}}\right) \mid A, B \in \Sigma, \mathbf{v} \in\{1, \ldots, \operatorname{arity}(B)+n\}^{\operatorname{arity}(B)},\right. \\
\left.\mathbf{w} \in\{0, \ldots, \operatorname{arity}(B)+n\}^{\operatorname{arity}(A)}, A\left(*_{B, \mathbf{v}}^{\mathbf{w}}\right) \neq B\left(*_{B, \mathbf{v}}^{\mathbf{v}}\right)\right\} \\
\text { - } \mathcal{D}_{r}^{\mathrm{f}_{0}}=\left\{B\left(*_{B, \mathbf{v}}^{\mathbf{v}}\right) \mid B \in \Sigma, \mathbf{v} \in\{1, \ldots, \operatorname{arity}(B)\}^{\operatorname{arity}(B)}\right\} \cup\{\perp\}
\end{gathered}
$$

We now show that the family $\Psi_{0}^{\mathrm{f}}$ is f-admissible. Consider a datalog rule $r=\varphi \rightarrow \gamma \in$ rel $_{\mathrm{f}(\mathcal{O}, \Sigma)}$. W.l.o.g. we can assume that $\gamma \notin \varphi$-otherwise $r$ would be tautological and hence entailed by any ontology. Let $\sigma$ be a substitution mapping all variables in $r$ to distinct fresh constants. Since $\gamma \notin \varphi$ and $\sigma$ is injective, we also have $\gamma \sigma \notin \varphi \sigma$. The fact $\gamma \sigma$ must be of the form $B(\mathbf{c})$ with $B \in \Sigma$ (if $B=\perp$ then it would be $\mathbf{c}=\emptyset$ ) and consider an injective substitution mapping all constants in $\mathbf{c} \cup \operatorname{Ct}(\mathcal{O})$ to $\{1, \ldots$, arity $(B)+n\}$ and satisfying $c \eta=\operatorname{arity}(B)+i$ iff $c=c_{i} \in \operatorname{Ct}(\mathcal{O})$. Let $\tau$ be another substitution defined on all constants in $\operatorname{Sig}(\varphi \sigma \cup \gamma \sigma)$ and such that

$$
c \tau= \begin{cases}*_{B, \mathbf{c} \eta}^{c \eta} & \text { if } c \in \mathbf{c} \cup \mathrm{Ct}(\mathcal{O}) \\ *_{B, \mathbf{c} \eta}^{0} & \text { otherwise }\end{cases}
$$

Note that $\tau$ is compatible with $\theta^{f_{0}}$.

It is easy to see that $(\gamma \sigma) \tau=B\left(*_{B, \mathbf{c} \eta}^{\mathbf{c} \eta}\right) \in \mathcal{D}_{r}^{\mathrm{f}_{0}}$ since $\left.\mathbf{c} \eta \in\{1, \ldots, \operatorname{arity}(B)+n\}\right\}^{\operatorname{arity}(B)}$. On the other hand, each fact in $(\varphi \sigma) \tau$ must be of the form $A\left(*_{B, \eta(\mathbf{c})}^{\mathbf{w}}\right)$ with $A \in \Sigma$ and $\mathbf{w} \in\{0, \ldots, \operatorname{arity}(B)+n\}^{\operatorname{arity}(A)}$. Because $\gamma \sigma \notin \varphi \sigma$ and $\eta$ is injective, it is easy to see that $A\left(*_{B, \mathbf{c} \eta}^{\mathrm{w}}\right) \neq B\left(*_{B, \mathbf{c} \eta}^{\mathrm{c} \eta}\right)$. Consequently, $(\varphi \sigma) \nu \subseteq \mathcal{D}_{r}^{\mathrm{f}_{0}}$. By Theorem 23, it follows that $\mathcal{O} \models r$ iff $\mathcal{M}^{\Psi_{0}^{\mathrm{f}}(\mathcal{O}, \Sigma)} \models r$, hence $\Psi_{0}^{\mathrm{f}}$ is f-admissible.

Finally, consider $\mathcal{O}=\{A(x) \rightarrow B(x), B(x) \rightarrow A(x)\}$ and $\Sigma=\{A\}$. It is easy to see that $\mathcal{M}^{\Psi^{\mathrm{f}}(\mathcal{O}, \Sigma)}=\mathcal{O} \nsubseteq \emptyset=\mathcal{M}^{\Psi_{0}^{\mathrm{f}}(\mathcal{O}, \Sigma)}$, and thus $\Psi^{\mathrm{f}}$ is not f-optimal.

Proposition 80. The family $\Psi^{\mathrm{q}}$ is not q-optimal.

Proof. Consider an arbitrary but fixed pair of $\mathcal{O}$ and $\Sigma$. Let $\operatorname{Ct}(\mathcal{O})=\left\{c_{1}, \ldots, c_{n}\right\}$ and let $\left\{y_{1}, \ldots, y_{m}\right\}$ be the existentially quantified variables mentioned in $\mathcal{O}$, and $\left\{c_{y_{1}}, \ldots, c_{y_{m}}\right\}$ a corresponding set of fresh constants. Furthermore, for each predicate $B \in \Sigma$ and each array $\mathbf{v} \in\{1, \ldots, \operatorname{arity}(B)+n+m\}^{\operatorname{arity}(B)}$ consider a set $\left\{*_{B, \mathbf{v}}^{i} \mid 0 \leq i \leq \operatorname{arity}(B)+n+m\right\}$ of constants such that

1. $*_{B, \mathbf{v}}^{0}, \ldots, *_{B, \mathbf{v}}^{\text {arity }}(B)$ are fresh constants,

2. $*_{B, \mathbf{v}}^{\text {arity }}(B), \ldots, *_{B, \mathbf{v}}^{\text {arity }(B)+n}$ are such that $*_{B, \mathbf{v}}^{\text {arity }(B)+i}=c_{i} \in \mathrm{Ct}(\mathcal{O})$ for each $1 \leq i \leq n$, and

3. $*_{B, \mathbf{v}}^{\text {arity }}(B)+n+1, \ldots, * *_{B, \mathbf{v}}^{\operatorname{arity}(B)+n+m}$ are such that $*_{B, \mathbf{v}}^{\operatorname{arity}(B)+n+i}=c_{y_{i}}$ for each $1 \leq i \leq m$

Let $*_{B, \mathbf{v}}^{\mathbf{w}}$ denote the array $\left(*_{B, \mathbf{v}}^{w_{1}}, \ldots, *_{B, \mathbf{v}}^{w_{n}}\right)$ whenever $\mathbf{w}=\left(w_{1}, \ldots, w_{n}\right)$, and consider the uniform module setting family $\Psi_{0}^{\mathrm{q}}$ such that $\Psi_{0}^{\mathrm{q}}(\mathcal{O}, \Sigma)=\left\langle\theta^{\mathrm{q}_{0}}, \mathcal{D}_{0}^{\mathrm{q}_{0}}, \mathcal{D}_{r}^{\mathrm{q}_{0}}\right\rangle$ is as follows:

- $\theta^{\mathrm{q}_{0}}=\left\{y \mapsto c_{y} \mid y\right.$ existentially quantified in $\left.\mathcal{O}\right\} \cup\{c \mapsto c \mid c \in \operatorname{Ct}(\mathcal{O})\}$

- $\mathcal{D}_{0}^{\mathrm{q}_{0}}=\left\{A\left(* *_{B, \mathbf{v}}^{\mathbf{w}}\right) \mid A, B \in \Sigma, \mathbf{v} \in\{1, \ldots, \operatorname{arity}(B)+n+m\}^{\operatorname{arity}(B)}\right.$,

$$
\left.\mathbf{w} \in\{0,1, \ldots, \operatorname{arity}(B)+n\}^{\operatorname{arity}(A)}, A\left(*_{B, \mathbf{v}}^{\mathbf{w}}\right) \neq B\left(*_{B, \mathbf{v}}^{\mathbf{v}}\right)\right\}
$$


- $\mathcal{D}_{r}^{\mathbf{q}_{0}}=\left\{B\left(*_{B, \mathbf{v}}^{\mathbf{v}}\right) \mid B \in \Sigma, \mathbf{v} \in\{1, \ldots, \operatorname{arity}(B)+n+m\}^{\operatorname{arity}(B)}\right\} \cup\{\perp\}$

We now show that the family $\Psi_{0}^{\mathrm{q}}$ is q-admissible.

Consider a rule $r=\varphi(\mathbf{x}) \rightarrow \exists \mathbf{y} \psi(\mathbf{x}, \mathbf{y}) \in \operatorname{rel}_{\mathbf{q}}(\mathcal{O}, \Sigma)$, a justification $\mathcal{O}^{\prime}$ of $r$ in $\mathcal{O}$ and a rule $s \in \mathcal{O}^{\prime}$. To show that $\Psi_{0}^{\mathrm{q}}(\mathcal{O}, \Sigma)$ is q-admissible it suffices to show that $s \subseteq \mathcal{M}^{\Psi_{0}^{\mathrm{q}}(\mathcal{O}, \Sigma)}$.

We can assume w.l.o.g. that $\perp \notin \varphi$ (otherwise $r$ would be tautological) and $\psi=\bigvee_{i=1}^{n} \psi_{i}$ with $m>0$ and each $\psi_{i}$ a conjunction of atoms. Similarly to the proof of Theorem 23, consider a fresh predicate $Q$ and the ontology

$$
\mathcal{O}_{Q}=\left\{\psi_{i}(\mathbf{x}, \mathbf{y}) \rightarrow Q(\mathbf{x}) \mid 1 \leq i \leq n\right\}
$$

as well as a module setting $\chi_{Q}=\left\langle\theta^{\mathbf{q}_{0}}, \mathcal{D}_{0}^{Q}, \mathcal{D}_{r}^{Q}\right\rangle$ for $\mathcal{O} \cup \mathcal{O}_{Q}$ and $\Sigma$, satisfying in particular $\mathcal{P}^{\chi Q}=\mathcal{P}^{\Psi_{0}^{\mathrm{q}}(\mathcal{O}, \Sigma)} \cup \mathcal{O}_{Q}$. As argued in the proof of Theorem 23, given $s \in \mathcal{O}^{\prime}$ and a substitution $\sigma$ mapping variables in $\mathbf{x}$ to fresh distinct constants, there must be a proof $\rho=(T, \lambda)$ of $Q(\mathbf{x}) \sigma$ in $\kappa\left(\mathcal{O} \cup \mathcal{O}_{Q}\right) \cup \varphi \sigma$ such that $\kappa(s) \cap \operatorname{supp}(\rho) \neq \emptyset$. Furthermore, thanks to $\mathcal{O}^{\prime}$ being a justification, we can assume that $\rho$ is laconic, and in particular for each leaf node $v \in T$ and each ancestor $w$ of $v$ in $T$ it must be $\lambda(v) \nsubseteq \lambda(w)$.

By Lemma 21, either $s=\perp \rightarrow \square$ or there exists a proof $\rho^{\prime}=\left(T^{\prime}, \lambda^{\prime}\right)$ in $\mathcal{P}^{\Psi_{0}^{\mathrm{q}}(\mathcal{O}, \Sigma)} \cup$ $(\varphi \sigma) \theta^{\mathrm{q}_{0}}$ of either $Q(\mathbf{x}) \sigma$ or $\perp$ that is embeddable into $\rho$ modulo $\theta^{\mathrm{q}_{0}}$ and such that $\operatorname{supp}\left(\rho^{\prime}\right) \cap$ $\Xi^{\Psi_{0}^{\mathrm{q}}(\mathcal{O}, \Sigma)}(s) \neq \emptyset$.

If $s=\perp \rightarrow \square$ then there must be some rule $s^{\prime}$ in $\mathcal{O}^{\prime}$ such that $\perp \in \operatorname{Sig}\left(\mathcal{O}^{\prime}\right)$. As we show next, when considering the case $s \neq \perp \rightarrow \square$, it must be $s^{\prime} \in \mathcal{M}^{\chi}$. Therefore $\perp \in \operatorname{Sig}\left(\mathcal{M}^{\chi}\right)$, and consequently $s=\perp \rightarrow \square \in \mathcal{M}^{\chi}$.

Otherwise, if $\rho^{\prime}=(T, \lambda)$ is a proof of $Q(\mathbf{x}) \sigma$, as discussed in the proof of Theorem 23, a rule from $\Xi^{\Psi_{0}^{\mathrm{q}}(\mathcal{O}, \Sigma)}(r)$ must be used in some subproof $\rho^{\prime \prime}=\left(T^{\prime \prime}, \lambda^{\prime \prime}\right)$ of $\rho^{\prime}$ that is a proof of $\left(\gamma \sigma^{\prime}\right) \theta^{q_{0}}$ for some $\gamma \in \bigcup_{i} \psi_{i}$ and some extension $\sigma^{\prime}$ of $\sigma$ to $\mathbf{y}$. Let $v$ be the root of $T^{\prime \prime}$ and $w_{1}, \ldots, w_{n}$ its leaves, by definition it must be $\lambda^{\prime \prime}(v)=\left(\gamma \sigma^{\prime}\right) \theta^{\mathrm{q}_{0}}$.

If $v$ is also a leaf in $T^{\prime \prime}$ then there must be a rule of the form $\left(\rightarrow\left(\gamma \sigma^{\prime}\right) \theta^{\mathrm{q}_{0}}\right)$ in $\Xi^{\Psi_{0}^{\mathrm{q}}(\mathcal{O}, \Sigma)}(s)$, and all the terms in $\left(\gamma \sigma^{\prime}\right) \theta^{\mathrm{q}_{0}}$ must be constants from the domain of $\theta^{\mathrm{q}_{0}}$. This implies $\left(\gamma \sigma^{\prime}\right) \theta^{\mathrm{q}_{0}} \in \mathcal{D}_{r}^{\mathrm{q}_{0}}$, and consequently $\left(\rightarrow\left(\gamma \sigma^{\prime}\right) \theta^{\mathrm{q}_{0}}\right) \in \operatorname{supp}\left(\Psi_{0}^{\mathrm{q}}(\mathcal{O}, \Sigma)\right)$ and $s \in \mathcal{M}^{\Psi_{0}^{\mathrm{q}}(\mathcal{O}, \Sigma)}$.

Suppose $v$ is not a leaf of $T^{\prime \prime}$. First of all, note that $(\varphi \sigma) \theta^{\mathrm{q}_{0}}=\varphi \sigma$ due to $\theta_{0}^{\mathrm{q}}$ not modifying constants and $\varphi \sigma$ not using functional terms, and therefore $\lambda^{\prime \prime}\left(w_{i}\right) \in \varphi \sigma$ for each $i$. If there are functional terms in $\gamma \sigma^{\prime}$ then $\left(\gamma \sigma^{\prime}\right) \theta^{\mathrm{q}_{0}} \notin \varphi \sigma$, since both the constants $c_{y}$ and the constants in the range of $\sigma$ were fresh by hypothesis, and therefore $\lambda^{\prime \prime}\left(w_{i}\right) \neq \lambda^{\prime \prime}(v)$ for each $i$. Suppose now that there are no functional terms in $\gamma \sigma^{\prime}$. Then $\gamma \sigma^{\prime} \theta^{\mathrm{q}_{0}}=\gamma \sigma^{\prime}$. We know that $\rho^{\prime}$, and hence $\rho^{\prime \prime}$, is embeddable into $\rho$ modulo $\theta^{\mathrm{q}_{0}}$. It follows that there must exist some node $v^{\prime} \in T$ that is not a leaf of $T$ and such that $\lambda(v) \subseteq \lambda\left(v^{\prime}\right) \theta^{\mathrm{q}_{0}}$, and also a collection of leaves $w_{1}^{\prime}, \ldots, w_{n}^{\prime}$ of $T$ such that $\lambda^{\prime \prime}\left(w_{i}\right)=\lambda\left(w_{i}^{\prime}\right) \theta^{\mathrm{q}_{0}} \in \varphi \sigma \theta^{\mathrm{q}_{0}}$. Since neither $\gamma \sigma^{\prime}$ nor $\varphi \sigma^{\prime}$ use functional terms, and $\theta_{0}^{\mathrm{q}}$ does not modify constants, it must in fact be $\lambda(v) \subseteq \lambda\left(v^{\prime}\right)$ and $\lambda^{\prime \prime}\left(w_{i}\right)=\lambda\left(w_{i}^{\prime}\right)$ for each $i$. Furthermore, we had that $\lambda\left(w_{i}\right) \nsubseteq \subset \lambda\left(v^{\prime}\right)$ for each $i$, which implies that, also in this case $\lambda^{\prime \prime}\left(w_{i}\right) \neq \lambda^{\prime \prime}(v)$ for each $i$. Therefore, regardless of $\gamma \sigma^{\prime}$ mentioning functional terms, we have $\lambda^{\prime \prime}\left(w_{i}\right) \neq \lambda^{\prime \prime}(v)$ for each $i$. Now, the fact $\gamma \sigma^{\prime}$ must be of the form $B(\mathbf{c})$ with $B \in \Sigma$ since $\gamma \in \bigcup_{i} \psi_{i}$ and by hypothesis $r \in \operatorname{rel}_{\mathbf{q}}(\mathcal{O}, \Sigma)$. Let $\eta$ be an injective substitution mapping all constants in $\mathbf{c} \cup \operatorname{range}(\theta)$ to $\{1, \ldots$, arity $(B)+n+m\}$ satisfying (i) $c \eta=\operatorname{arity}(B)+i$ iff $c=c_{i} \in \operatorname{Ct}(\mathcal{O})$ and (ii) $c \eta=\operatorname{arity}(B)+n+i$ iff $c=c_{y_{i}}$. Let $\tau$ be 
another substitution defined on all constants mentioned by $\rho^{\prime \prime}$ and such that

$$
c \tau= \begin{cases}*_{B, \mathbf{c} \eta}^{c \eta} & \text { if } c \in \mathbf{c} \cup \operatorname{range}(\theta) \\ *_{B, \mathbf{c} \eta}^{0} & \text { otherwise }\end{cases}
$$

Note that $\tau$ is compatible with $\theta$. Since $\mathbf{c} \eta \in\{1, \ldots \text {, arity }(B)+n+m\}^{\operatorname{arity}(B)}$, it is easy to see that $\lambda^{\prime \prime}(v) \tau=B\left(*_{B, \mathbf{c} \eta}^{\mathbf{c} \eta}\right) \in \mathcal{D}_{r}^{\mathrm{f}_{0}}$. On the other hand, as we have previously observed, no $\lambda^{\prime \prime}\left(w_{i}\right)$ mentions constants from the set $\left\{c_{y_{1}}, \ldots, c_{y_{m}}\right\}$, hence $\lambda^{\prime \prime}\left(w_{i}\right) \tau$ must be of the form $A\left(*_{B, \eta(\mathbf{c})}^{\mathbf{w}}\right)$ with $A \in \Sigma$ and $\mathbf{w} \in\{0, \ldots, \operatorname{arity}(B)+n\}^{\text {arity }(A)}$. Furthermore, because $\lambda^{\prime \prime}\left(w_{i}\right) \neq \lambda^{\prime \prime}(v)$ for each $i$, it follows that also $\lambda^{\prime \prime}\left(w_{i}\right) \tau \neq \lambda^{\prime \prime}(v) \tau$ and thus $\lambda^{\prime \prime}\left(w_{i}\right) \tau \in \mathcal{D}_{0}^{f_{0}}$ for each $i$. The proof $\rho^{\prime \prime} \tau=\left(T^{\prime \prime}, \lambda_{\tau}^{\prime \prime}\right)$ such that $\lambda_{\tau}^{\prime \prime}(v)=\left(\lambda^{\prime \prime}(v)\right) \tau$ is clearly a proof in $\mathcal{P}^{\Psi_{0}^{\mathrm{q}}(\mathcal{O}, \Sigma)} \cup \mathcal{D}_{0}^{\mathrm{q}_{0}}$ of $\lambda^{\prime \prime}(v) \tau$ that has the same support as $\rho^{\prime \prime}$, therefore $s \in \mathcal{M}^{\Psi_{0}^{\mathrm{q}}(\mathcal{O}, \Sigma)}$.

Finally, if $\rho^{\prime}$ is a proof of $\perp$ then it must be a proof in $\mathcal{P}^{\Psi_{0}^{\mathrm{q}}(\mathcal{O}, \Sigma)}$. Since by assumption it is $\perp \notin \varphi$, the labels of all the leaves in $\rho^{\prime}$ must also be different from $\perp$. To check that also in this case $s \in \mathcal{M}^{\Psi_{0}^{\mathrm{q}}(\mathcal{O}, \Sigma)}$, it suffices to follow a similar argument to the one above with a mapping $\tau^{\prime}$ defined on all constants mentioned in $\rho^{\prime}$ and such that

$$
c \tau= \begin{cases}c & \text { if } c \in \mathrm{Ct}(\mathcal{O}) \cup\left\{c_{y_{1}}, \ldots, c_{y_{m}}\right\} \\ *_{B, \mathbf{c} \eta}^{0} & \text { otherwise }\end{cases}
$$

We have proved that the family $\Psi_{0}^{\mathrm{q}}$ is q-admissible. Now we will use it to show that $\Psi^{\mathrm{q}}$ is not q-optimal. As in the proof for Theorem 79 , if we consider the ontology $\mathcal{O}=\{A(x) \rightarrow B(x), B(x) \rightarrow A(x)\}$ and the signature $\Sigma=\{A\}$, we can observe that $\mathcal{M}^{\Psi^{\mathrm{q}}(\mathcal{O}, \Sigma)}=\mathcal{O} \nsubseteq \emptyset=\mathcal{M}^{\Psi_{0}^{\mathrm{q}}(\mathcal{O}, \Sigma)}$, and thus $\Psi^{\mathrm{q}}$ is not q-optimal.

\section{References}

Abiteboul, S., Hull, R., \& Vianu, V. (1995). Foundations of Databases. Addison-Wesley.

Alviano, M., Faber, W., Leone, N., \& Manna, M. (2012). Disjunctive datalog with existential quantifiers: Semantics, decidability, and complexity issues. Theory and Practice of Logic Programming, 12(4-5), 701-718.

Amir, E., \& McIlraith, S. A. (2005). Partition-based logical reasoning for first-order and propositional theories. Artificial Intelligence, 162(1-2), 49-88.

Antoniou, G., \& Kehagias, A. (2000). A note on the refinement of ontologies. International Journal of Intelligent Systems, 15(7), 623-632.

Armas Romero, A., Cuenca Grau, B., \& Horrocks, I. (2012). MORe: Modular combination of OWL reasoners for ontology classification. In Cudré-Mauroux, P., Heflin, J., Sirin, E., Tudorache, T., Euzenat, J., Hauswirth, M., Parreira, J. X., Hendler, J., Schreiber, G., Bernstein, A., \& Blomqvist, E. (Eds.), Proceedings of the 11th International Semantic Web Conference, Part I, Vol. 7649 of Lecture Notes in Computer Science, pp. 1-16. Springer.

Armas Romero, A., Kaminski, M., Cuenca Grau, B., \& Horrocks, I. (2015). Ontology module extraction via datalog reasoning. In Bonet, B., \& Koenig, S. (Eds.), Proceedings of the 29th AAAI Conference on Artificial Intelligence, pp. 1410-1416. AAAI Press. 
Baader, F., Bienvenu, M., Lutz, C., \& Wolter, F. (2010). Query and predicate emptiness in description logics. In Lin, F., Sattler, U., \& Truszczynski, M. (Eds.), Proceedings of the 12th International Conference on Principles of Knowledge Representation and Reasoning. AAAI Press.

Baader, F., Brandt, S., \& Lutz, C. (2005). Pushing the $\mathcal{E} \mathcal{L}$ envelope. In Kaelbling, L. P., \& Saffiotti, A. (Eds.), Proceedings of the 19th International Joint Conference on Artificial Intelligence, pp. 364-369. Professional Book Center.

Baader, F., Calvanese, D., McGuinness, D. L., Nardi, D., \& Patel-Schneider, P. F. (Eds.). (2003). The Description Logic Handbook: Theory, Implementation, and Applications. Cambridge University Press.

Bachmair, L., \& Ganzinger, H. (2001). Resolution theorem proving. In Robinson, J. A., \& Voronkov, A. (Eds.), Handbook of Automated Reasoning, pp. 19-99. Elsevier and MIT Press.

Botoeva, E., Kontchakov, R., Ryzhikov, V., Wolter, F., \& Zakharyaschev, M. (2014). Query inseparability for description logic knowledge bases. In Baral, C., Giacomo, G. D., \& Eiter, T. (Eds.), Proceedings of the 14th International Conference on Principles of Knowledge Representation and Reasoning. AAAI Press.

Bourhis, P., Morak, M., \& Pieris, A. (2013). The impact of disjunction on query answering under guarded-based existential rules. In Rossi, F. (Ed.), Proceedings of the 23rd International Joint Conference on Artificial Intelligence. IJCAI/AAAI.

Bry, F., Eisinger, N., Eiter, T., Furche, T., Gottlob, G., Ley, C., Linse, B., Pichler, R., \& Wei, F. (2007). Foundations of rule-based query answering. In Antoniou, G., Aßmann, U., Baroglio, C., Decker, S., Henze, N., Patranjan, P., \& Tolksdorf, R. (Eds.), Proceedings of the 3rd International Reasoning Web Summer School, Tutorial Lectures, Vol. 4636 of Lecture Notes in Computer Science, pp. 1-153. Springer.

Calì, A., Gottlob, G., Lukasiewicz, T., Marnette, B., \& Pieris, A. (2010). Datalog+/-: A family of logical knowledge representation and query languages for new applications. In Proceedings of the 25th Annual ACM/IEEE Symposium on Logic in Computer Science, pp. 228-242. IEEE Computer Society.

Chekuri, C., \& Rajaraman, A. (2000). Conjunctive query containment revisited. Theoretical Computer Science, 239(2), 211-229.

Cuenca Grau, B., Halaschek-Wiener, C., Kazakov, Y., \& Suntisrivaraporn, B. (2010). Incremental classification of description logics ontologies. Journal of Automated Reasoning, $44(4), 337-369$.

Cuenca Grau, B., Horrocks, I., Kazakov, Y., \& Sattler, U. (2007a). Just the right amount: Extracting modules from ontologies. In Williamson, C. L., Zurko, M. E., PatelSchneider, P. F., \& Shenoy, P. J. (Eds.), Proceedings of the 16th International World Wide Web Conference, pp. 717-726. ACM.

Cuenca Grau, B., Horrocks, I., Kazakov, Y., \& Sattler, U. (2007b). A logical framework for modularity of ontologies. In Veloso, M. M. (Ed.), Proceedings of the 20th International Joint Conference on Artificial Intelligence, pp. 298-303. 
Module Extraction in Expressive Ontology Languages via Datalog Reasoning

Cuenca Grau, B., Horrocks, I., Kazakov, Y., \& Sattler, U. (2008). Modular reuse of ontologies: Theory and practice. Journal of Artificial Intelligence Research, 31, 273-318.

Cuenca Grau, B., Horrocks, I., Krötzsch, M., Kupke, C., Magka, D., Motik, B., \& Wang, Z. (2013). Acyclicity notions for existential rules and their application to query answering in ontologies. Journal of Artificial Intelligence Research, 47, 741-808.

Cuenca Grau, B., Horrocks, I., Motik, B., Parsia, B., Patel-Schneider, P. F., \& Sattler, U. (2008). OWL 2: The next step for OWL. Journal of Web Semantics, 6(4), 309-322.

Dantsin, E., Eiter, T., Gottlob, G., \& Voronkov, A. (2001). Complexity and expressive power of logic programming. ACM Computing Surveys, 33(3), 374-425.

Del Vescovo, C., Klinov, P., Parsia, B., Sattler, U., Schneider, T., \& Tsarkov, D. (2013). Empirical study of logic-based modules: Cheap is cheerful. In Alani, H., Kagal, L., Fokoue, A., Groth, P. T., Biemann, C., Parreira, J. X., Aroyo, L., Noy, N. F., Welty, C., \& Janowicz, K. (Eds.), Proceedings of the 12th International Semantic Web Conference, Part I, Vol. 8218 of Lecture Notes in Computer Science, pp. 84-100. Springer.

Del Vescovo, C., Parsia, B., Sattler, U., \& Schneider, T. (2011). The modular structure of an ontology: Atomic decomposition. In Walsh, T. (Ed.), Proceedings of the 22nd International Joint Conference on Artificial Intelligence, pp. 2232-2237. IJCAI/AAAI.

Eiter, T., Ianni, G., Schindlauer, R., Tompits, H., \& Wang, K. (2006). Forgetting in managing rules and ontologies. In Proceedings of the 2006 IEEE / WIC / ACM International Conference on Web Intelligence, pp. 411-419. IEEE Computer Society.

Gatens, W., Konev, B., \& Wolter, F. (2014). Lower and upper approximations for depleting modules of description logic ontologies. In Schaub, T., Friedrich, G., \& O'Sullivan, B. (Eds.), Proceedings of the 21st European Conference on Artificial Intelligence, Vol. 263 of Frontiers in Artificial Intelligence and Applications, pp. 345-350. IOS Press.

Ghilardi, S., Lutz, C., \& Wolter, F. (2006a). Did I damage my ontology? A case for conservative extensions in description logics. In Doherty, P., Mylopoulos, J., \& Welty, C. A. (Eds.), Proceedings of the 10th International Conference on Principles of Knowledge Representation and Reasoning, pp. 187-197. AAAI Press.

Ghilardi, S., Lutz, C., Wolter, F., \& Zakharyaschev, M. (2006b). Conservative extensions in modal logic. In Governatori, G., Hodkinson, I. M., \& Venema, Y. (Eds.), Proceedings of the 6th Advances in Modal Logic Conference, pp. 187-207. College Publications.

Glimm, B., Horrocks, I., Motik, B., Stoilos, G., \& Wang, Z. (2014). HermiT: An OWL 2 reasoner. Journal of Automated Reasoning, 53(3), 245-269.

Grohe, M., Schwentick, T., \& Segoufin, L. (2001). When is the evaluation of conjunctive queries tractable?. In Vitter, J. S., Spirakis, P. G., \& Yannakakis, M. (Eds.), Proceedings of the 33rd Annual ACM Symposium on Theory of Computing, pp. 657-666. ACM.

Horridge, M., \& Bechhofer, S. (2011). The OWL API: A java API for OWL ontologies. Semantic Web, 2(1), 11-21.

Horridge, M., Parsia, B., \& Sattler, U. (2008). Laconic and precise justifications in OWL. In Sheth, A. P., Staab, S., Dean, M., Paolucci, M., Maynard, D., Finin, T. W., \& 
Thirunarayan, K. (Eds.), Proceedings of the 7th International Semantic Web Conference, Vol. 5318 of Lecture Notes in Computer Science, pp. 323-338. Springer.

Horrocks, I., Kutz, O., \& Sattler, U. (2006). The even more irresistible $\mathcal{S} \mathcal{R O} \mathcal{I} \mathcal{Q}$. In Doherty, P., Mylopoulos, J., \& Welty, C. A. (Eds.), Proceedings of the 10th International Conference on Principles of Knowledge Representation and Reasoning, pp. 57-67. AAAI Press.

Horrocks, I., Patel-Schneider, P. F., \& van Harmelen, F. (2003). From SHIQ and RDF to OWL: the making of a web ontology language. Journal of Web Semantics, 1(1), 7-26.

Jiménez-Ruiz, E., \& Cuenca Grau, B. (2011). LogMap: Logic-based and scalable ontology matching. In Aroyo, L., Welty, C., Alani, H., Taylor, J., Bernstein, A., Kagal, L., Noy, N. F., \& Blomqvist, E. (Eds.), Proceedings of the 10th International Semantic Web Conference, Part I, Vol. 7031 of Lecture Notes in Computer Science, pp. 273-288. Springer.

Jiménez-Ruiz, E., Cuenca Grau, B., Horrocks, I., \& Berlanga Llavori, R. (2011). Supporting concurrent ontology development: Framework, algorithms and tool. Data $\&$ Knowledge Engineering, 70(1), 146-164.

Jiménez-Ruiz, E., Cuenca Grau, B., Sattler, U., Schneider, T., \& Berlanga Llavori, R. (2008). Safe and economic re-use of ontologies: A logic-based methodology and tool support. In Bechhofer, S., Hauswirth, M., Hoffmann, J., \& Koubarakis, M. (Eds.), Proceedings of the 5th European Semantic Web Conference, Vol. 5021 of Lecture Notes in Computer Science, pp. 185-199. Springer.

Kalyanpur, A., Parsia, B., Horridge, M., \& Sirin, E. (2007). Finding all justifications of OWL DL entailments. In Aberer, K., Choi, K., Noy, N. F., Allemang, D., Lee, K., Nixon, L. J. B., Golbeck, J., Mika, P., Maynard, D., Mizoguchi, R., Schreiber, G., \& CudréMauroux, P. (Eds.), Proceedings of the 6th International Semantic Web Conference and 2nd Asian Semantic Web Conference, Vol. 4825 of Lecture Notes in Computer Science, pp. 267-280. Springer.

Kalyanpur, A., Parsia, B., Sirin, E., \& Cuenca Grau, B. (2006). Repairing unsatisfiable concepts in OWL ontologies. In Sure, Y., \& Domingue, J. (Eds.), Proceedings of the 3rd European Semantic Web Conference, Vol. 4011 of Lecture Notes in Computer Science, pp. 170-184. Springer.

Kalyanpur, A., Parsia, B., Sirin, E., \& Hendler, J. A. (2005). Debugging unsatisfiable classes in OWL ontologies. Journal of Web Semantics, 3(4), 268-293.

Konev, B., Kontchakov, R., Ludwig, M., Schneider, T., Wolter, F., \& Zakharyaschev, M. (2011). Conjunctive query inseparability of OWL 2 QL tboxes. In Burgard, W., \& Roth, D. (Eds.), Proceedings of the 25th AAAI Conference on Artificial Intelligence. AAAI Press.

Konev, B., Ludwig, M., Walther, D., \& Wolter, F. (2012). The logical difference for the lightweight description logic $\mathcal{E} \mathcal{L}$. Journal of Artificial Intelligence Research, 44, 633708.

Konev, B., Lutz, C., Ponomaryov, D. K., \& Wolter, F. (2010). Decomposing description logic ontologies. In Lin, F., Sattler, U., \& Truszczynski, M. (Eds.), Proceedings of 
the 12th International Conference on Principles of Knowledge Representation and Reasoning. AAAI Press.

Konev, B., Lutz, C., Walther, D., \& Wolter, F. (2009). Formal properties of modularisation. In Stuckenschmidt, H., Parent, C., \& Spaccapietra, S. (Eds.), Modular Ontologies: Concepts, Theories and Techniques for Knowledge Modularization, Vol. 5445 of Lecture Notes in Computer Science, pp. 25-66. Springer.

Konev, B., Lutz, C., Walther, D., \& Wolter, F. (2013). Model-theoretic inseparability and modularity of description logic ontologies. Artificial Intelligence, 203, 66-103.

Konev, B., Walther, D., \& Wolter, F. (2009). Forgetting and uniform interpolation in largescale description logic terminologies. In Boutilier, C. (Ed.), Proceedings of the 21st International Joint Conference on Artificial Intelligence, pp. 830-835.

Kontchakov, R., Lutz, C., Toman, D., Wolter, F., \& Zakharyaschev, M. (2011). The combined approach to ontology-based data access. In Walsh, T. (Ed.), Proceedings of the 22nd International Joint Conference on Artificial Intelligence, pp. 2656-2661. IJCAI/AAAI.

Kontchakov, R., Wolter, F., \& Zakharyaschev, M. (2010). Logic-based ontology comparison and module extraction, with an application to DL-Lite. Artificial Intelligence, 174(15), $1093-1141$.

Koopmann, P., \& Schmidt, R. A. (2014). Count and forget: Uniform interpolation of $\mathcal{S H} \mathcal{Q}-$ ontologies. In Demri, S., Kapur, D., \& Weidenbach, C. (Eds.), Proceedings of the 7 th International Joint Conference on Automated Reasoning, Vol. 8562 of Lecture Notes in Computer Science, pp. 434-448. Springer.

Krötzsch, M., Rudolph, S., \& Hitzler, P. (2008a). Description logic rules. In Ghallab, M., Spyropoulos, C. D., Fakotakis, N., \& Avouris, N. M. (Eds.), Proceedings of the 18th European Conference on Artificial Intelligence, Vol. 178 of Frontiers in Artificial Intelligence and Applications, pp. 80-84. IOS Press.

Krötzsch, M., Rudolph, S., \& Hitzler, P. (2008b). ELP: Tractable rules for OWL 2. In Sheth, A. P., Staab, S., Dean, M., Paolucci, M., Maynard, D., Finin, T. W., \& Thirunarayan, K. (Eds.), Proceedings of the 7th International Semantic Web Conference, Vol. 5318 of Lecture Notes in Computer Science, pp. 649-664. Springer.

Ludwig, M. (2014). Just: A tool for computing justifications w.r.t. $\mathcal{E} \mathcal{L H}$ ontologies. In Bail, S., Glimm, B., Jiménez-Ruiz, E., Matentzoglu, N., Parsia, B., \& Steigmiller, A. (Eds.), Proceedings of the 3rd International Workshop on OWL Reasoner Evaluation, Vol. 1207 of CEUR Workshop Proceedings, pp. 1-7. CEUR-WS.org.

Ludwig, M., \& Konev, B. (2014). Practical uniform interpolation and forgetting for $\mathcal{A L C}$ tboxes with applications to logical difference. In Baral, C., Giacomo, G. D., \& Eiter, T. (Eds.), Proceedings of the 14th International Conference on Principles of Knowledge Representation and Reasoning. AAAI Press.

Lutz, C., \& Wolter, F. (2010). Deciding inseparability and conservative extensions in the description logic EL. Journal of Symbolic Computation, 45(2), 194-228. 
Lutz, C., \& Wolter, F. (2011). Foundations for uniform interpolation and forgetting in expressive description logics. In Walsh, T. (Ed.), Proceedings of the 22nd International Joint Conference on Artificial Intelligence, pp. 989-995. IJCAI/AAAI.

Marnette, B. (2009). Generalized schema-mappings: From termination to tractability. In Paredaens, J., \& Su, J. (Eds.), Proceedings of the 28th ACM SIGMOD Symposium on Principles of Database Systems, pp. 13-22. ACM.

Motik, B. (2006). Reasoning in Description Logics Using Resolution and Deductive Databases. Ph.D. thesis, Univesität Karlsruhe (TH), Karlsruhe, Germany.

Motik, B., Nenov, Y., Piro, R., Horrocks, I., \& Olteanu, D. (2014). Parallel materialisation of datalog programs in centralised, main-memory RDF systems. In Brodley, C. E., \& Stone, P. (Eds.), Proceedings of the 28th AAAI Conference on Artificial Intelligence, pp. 129-137. AAAI Press.

Motik, B., Patel-Schneider, P. F., \& Parsia, B. (2012). OWL 2 web ontology language structural specification and functional-style syntax..

Nikitina, N., \& Rudolph, S. (2014). (Non-)succinctness of uniform interpolants of general terminologies in the description logic $\mathcal{E} \mathcal{L}$. Artificial Intelligence, 215, 120-140.

Nonnengart, A., \& Weidenbach, C. (2001). Computing small clause normal forms. In Robinson, J. A., \& Voronkov, A. (Eds.), Handbook of Automated Reasoning, pp. 335367. Elsevier and MIT Press.

Nortje, R., Britz, K., \& Meyer, T. (2012). A normal form for hypergraph-based module extraction for $\mathcal{S} \mathcal{R O} \mathcal{I} \mathcal{Q}$. In Gerber, A., Taylor, K., Meyer, T., \& Orgun, M. (Eds.), Proceedings of the 8th Australasian Ontology Workshop, Vol. 969 of CEUR Workshop Proceedings, pp. 40-51. CEUR-WS.org.

Nortje, R., Britz, K., \& Meyer, T. (2013). Reachability modules for the description logic $\mathcal{S R I}$ Q. In McMillan, K. L., Middeldorp, A., \& Voronkov, A. (Eds.), Proceedings of the 19th International Conference on Logic for Programming, Artificial Intelligence and Reasoning, Vol. 8312 of Lecture Notes in Computer Science, pp. 636-652. Springer.

Robinson, J. A. (1965). Automatic deduction with hyper-resolution. International Journal of Computer Mathematics, 1(3), 227-234.

Rousset, M.-C., \& Ulliana, F. (2015). Extracting bounded-level modules from deductive RDF triplestores. In Bonet, B., \& Koenig, S. (Eds.), Proceedings of the 29th AAAI Conference on Artificial Intelligence, pp. 268-274. AAAI Press.

Sattler, U., Schneider, T., \& Zakharyaschev, M. (2009). Which kind of module should I extract?. In Grau, B. C., Horrocks, I., Motik, B., \& Sattler, U. (Eds.), Proceedings of the 22nd International Workshop on Description Logics, Vol. 477 of CEUR Workshop Proceedings. CEUR-WS.org.

Schlicht, A., \& Stuckenschmidt, H. (2009). Distributed resolution for expressive ontology networks. In Polleres, A., \& Swift, T. (Eds.), Proceedings of the 3rd International Conference on Web Reasoning and Rule Systems, Vol. 5837, pp. 87-101. Springer.

Schlobach, S., \& Cornet, R. (2003). Non-standard reasoning services for the debugging of description logic terminologies. In Gottlob, G., \& Walsh, T. (Eds.), Proceedings of the 
18th International Joint Conference on Artificial Intelligence, pp. 355-362. Morgan Kaufmann.

Seidenberg, J., \& Rector, A. L. (2006). Web ontology segmentation: Analysis, classification and use. In Carr, L., Roure, D. D., Iyengar, A., Goble, C. A., \& Dahlin, M. (Eds.), Proceedings of the 15th International World Wide Web Conference, pp. 13-22. ACM.

Stefanoni, G., Motik, B., \& Horrocks, I. (2013). Introducing nominals to the combined query answering approaches for $\mathcal{E} \mathcal{L}$. In desJardins, M., \& Littman, M. L. (Eds.), Proceedings of the 27th AAAI Conference on Artificial Intelligence. AAAI Press.

Stuckenschmidt, H., Parent, C., \& Spaccapietra, S. (Eds.). (2009). Modular Ontologies: Concepts, Theories and Techniques for Knowledge Modularization, Vol. 5445 of Lecture Notes in Computer Science. Springer.

Suntisrivaraporn, B. (2008). Module extraction and incremental classification: A pragmatic approach for $\mathcal{E} \mathcal{L}+$ ontologies. In Bechhofer, S., Hauswirth, M., Hoffmann, J., \& Koubarakis, M. (Eds.), Proceedings of the 5th European Semantic Web Conference, Vol. 5021 of Lecture Notes in Computer Science, pp. 230-244. Springer.

Suntisrivaraporn, B., Qi, G., Ji, Q., \& Haase, P. (2008). A modularization-based approach to finding all justifications for OWL DL entailments. In Domingue, J., \& Anutariya, C. (Eds.), Proceedings of the 3rd Asian Semantic Web Conference, Vol. 5367 of Lecture Notes in Computer Science, pp. 1-15. Springer.

Tsarkov, D., \& Palmisano, I. (2012). Chainsaw: A metareasoner for large ontologies. In Horrocks, I., Yatskevich, M., \& Jiménez-Ruiz, E. (Eds.), Proceedings of the 1st International Workshop on OWL Reasoner Evaluation, Vol. 858 of CEUR Workshop Proceedings. CEUR-WS.org.

W3C OWL Working Group (2012). OWL 2 web ontology language document overview (second edition). W3C recommendation, World Wide Web Consortium.

Wang, K., Wang, Z., Topor, R. W., Pan, J. Z., \& Antoniou, G. (2014). Eliminating concepts and roles from ontologies in expressive descriptive logics. Computational Intelligence, $30(2), 205-232$.

Wang, Z., Wang, K., Topor, R. W., \& Pan, J. Z. (2010). Forgetting for knowledge bases in DL-Lite. Annals of Mathematics and Artificial Intelligence, 58(1-2), 117-151.

Zhou, Y., Cuenca Grau, B., Horrocks, I., Wu, Z., \& Banerjee, J. (2013). Making the most of your triple store: Query answering in OWL 2 using an RL reasoner. In Schwabe, D., Almeida, V. A. F., Glaser, H., Baeza-Yates, R. A., \& Moon, S. B. (Eds.), Proceedings of the 22nd International World Wide Web Conference, pp. 1569-1580. International World Wide Web Conferences Steering Committee / ACM.

Zhou, Y., Cuenca Grau, B., Nenov, Y., Kaminski, M., \& Horrocks, I. (2015). PAGOdA: Pay-as-you-go ontology query answering using a datalog reasoner. Journal of Artificial Intelligence Research, 54, 309-367.

Zhou, Y., Nenov, Y., Cuenca Grau, B., \& Horrocks, I. (2013). Complete query answering over horn ontologies using a triple store. In Alani, H., Kagal, L., Fokoue, A., Groth, P. T., Biemann, C., Parreira, J. X., Aroyo, L., Noy, N. F., Welty, C., \& Janowicz, K. 
Armas Romero, Kaminski, Cuenca Grau, \& Horrocks

(Eds.), Proceedings of the 12th International Semantic Web Conference, Part I, Vol. 8218 of Lecture Notes in Computer Science, pp. 720-736. Springer.

Zhou, Y., Nenov, Y., Cuenca Grau, B., \& Horrocks, I. (2014). Pay-as-you-go OWL query answering using a triple store. In Brodley, C. E., \& Stone, P. (Eds.), Proceedings of the 28th AAAI Conference on Artificial Intelligence, pp. 1142-1148. AAAI Press. 